\title{
DEVELOPMENT OF ADVANCED \\ ACQUISITION AND RECONSTRUCTION TECHNIQUES FOR REAL-TIME PERFUSION MRI
}

\author{
Dissertation \\ for the award of the degree \\ "Doctor rerum naturalium" \\ of the Georg-August-Universität Göttingen \\ within the doctoral program \\ Physics of Biological and Complex Systems \\ of the Georg-August University School of Science (GAUSS) \\ submitted by \\ VOLKERT BRAR ROELOFFS \\ from Wyk auf Föhr, Germany \\ Göttingen 2016
}


THESIS COMMITTEE

FIRST REFEREE AND SUPERVISOR

Prof. Dr. Jens Frahm

Biomedizinische NMR Forschungs GmbH

Max-Planck-Institut für biophysikalische Chemie, Göttingen

SECOND REFEREE

Prof. Dr. Marina Bennati

Forschungsgruppe EPR-Spektroskopie

Max-Planck-Institut für biophysikalische Chemie, Göttingen

Prof. Dr. Tim Salditt

Institut für Röntgenphysik

Georg-August-Universität Göttingen

EXTERNAL REFEREES

THIRD REFEREE

Prof. Dr. Michael Bock

Experimentelle Radiologie

Universitätsklinikum Freiburg

MEMBERS OF THE EXAMINATION BOARD

Prof. Dr. Jens Frahm

Prof. Dr. Marina Bennati

PD Dr. Peter Dechent

Medizinische Fakultät

Georg-August-Universität Göttingen

Apl. Prof. Dr. Ulrich Parlitz

Forschungsgruppe biomedizinische Physik

Max-Planck-Institut für Dynamik und Selbstorganization, Göttingen

Date of oral examination: 16 June 2016 
Here I declare that my doctoral thesis entitled "Development of Advanced Acquisition and Reconstruction Techniques for Real-Time Perfusion MRI" has been written independently with no other sources or aids than quoted.

Volkert Brar Roeloffs

Göttingen, April 2016 

Uun toochter am Ricklef 



\section{ACKNOWLEDGMENTS}

Die Gelegenheit, Danke zu sagen, habe ich zu oft schon ungenutzt verstreichen lassen. Deshalb packe ich sie jetzt am Schopfe:

Größter Dank gebührt Prof. Jens Frahm, der mich mit seiner Biomedizinischen NMR Forschungs GmbH nach Göttingen "gelockt" hat. Mit ihm zu arbeiten und $\mathrm{zu}$ forschen, hat mir außerordentlich viel Spaß gemacht. In meiner Arbeit hat er mir stets den nötigen akademischen Freiraum gelassen und mich gleichzeitig mit vollem Einsatz beim Verfassen von Manuskripten, dem Vorbereiten von Präsentationen und in fachlichen Fragen kompetent und kontinuierlich unterstützt.

Des Weiteren bin ich froh, mit Prof. Marina Bennati, Leiterin der Forschungsgruppe „Elektronenspinresonanz-Spektroskopie“ , nicht nur eine kompetente Zweitgutachterin aus einem verwandten Gebiet gefunden zu haben, sondern auch ein weiteres Mitglied in meiner Dissertationskommission.

Prof. Tim Salditt vom Institut für Röntgenphysik an der Universität Göttingen, möchte ich dafür danken, dass er meine Doktorarbeit als drittes Mitglied in meiner Dissertationskommission betreut hat.

Bei Prof. Christina Unterberg-Buchwald von der Universitätsmedizin Göttingen möchte ich mich zum einen für den spannenden Einblick in tägliche Untersuchungsroutinen bedanken, zum anderen aber auch für die Unterstützung bei der Kontrastmittelversorgung.

Dr. Kai Kallenberg (ebenfalls Universitätsmedizin Göttingen) hat uns schnell und unkompliziert einen klinischen Kontrastmittelinjektor zur Verfügung gestellt. Dafür noch einmal ein herzliches Dankeschön.

Mit "meinem“ Masterstudenten Jost Kollmeier habe ich - so scheint es mir außerordentliches Glück gehabt. Sein kluger Kopf und sein handwerkliches Geschick haben maßgeblich zum Gelingen der Perfusionsexperimente beigetragen. Danke für die vielen aufschlussreichen Diskussionen und die amüsanten Stunden am Scanner! 
Bei all meinen aktuellen und ehemaligen Kollegen aus der BiomedNMR möchte ich mich herzlich für die entspannte Arbeitsatmosphäre in unsere Gruppe bedanken. Ich habe mich von Anfang sehr wohl bei euch gefühlt. Besonders die täglichen Treffen an der ",achtarmigen Krake” im Keller werde ich in guter Erinnerung behalten.

Danken möchte ich insbesondere Sylke, die mir in allen administrativen Fragen kompetent zur Seite stand, und Kurt, der mich in Sachen IT bestens unterstützt hat.

Meinem Arbeitskollegen Xiaoqing, mit dem ich lange das Büro geteilt habe, möchte ich für die unzähligen Diskussionen und die gute Zusammenarbeit danken. Die Wissenschaft scheint eine stabile Brücke zwischen den Kulturen zu sein.

Auf Markus war nicht nur beim Debugging Verlass, sondern auch in der kanadischen Wildnis. Danke!

Mein Dank gilt auch Renate, weil ich das Gefühl nicht loswerde, mein Wohlergehen liege ihr irgendwie am Herzen. Konkret möchte ich Danke sagen, dass sie mir rechtzeitig die verschiedenen Formen vor Augen geführt hat, die eine Dissertationsschrift annehmen kann.

Frauke Bergmann, Antje Erdmann und Tina Trost von der IMPRS-Koordinationsstelle möchte ich Danke sagen, weil sie mir während meiner Dissertation in allen Fragen zu Bestätigungen, Bescheinigungen, Fristen und Regularien freundlich und schnell weiterhelfen konnten.

Meiner Graduiertenschule IMPRS-PBCS verdanke ich vier Reisekostenstipendien und somit all die Möglichkeiten, die nationale und internationale Konferenzen jungen Doktoranden bieten. Danke auch dafür!

Irene Böttcher-Gajewski vom Medienservice unseres Instituts gilt mein Dank für die professionellen Aufnahmen unseres Equipments. Ich habe immer noch ein schlechtes Gewissen, dass unsere vermeintlich kurze Fotosession so ausgeufert ist.

Besonderer Dank gilt auch meinen Probanden, die ich leider nicht namentlich nennen darf. Ohne sie wäre diese Art der angewandten Forschung nicht denkbar.

Der Firma Medicor gilt mein Dank, weil sie uns kostenlos zwei Leerspritzen für Forschungszwecke zur Verfügung gestellt hat. 
Den - hoffentlich erfolgreichen - Kampf gegen Fehlerteufel, Stilblüten und Ungereimtheiten haben für mich Patricia, Sabine, Christian, Jakob und Markus geführt. Sollte der geneigte Leser also wider Erwarten Tippfehler oder Zahlendreher finden, so sei hiermit der Verantwortungsbereich klar abgesteckt ;)

Der sportliche Ausgleich neben all der Schreibtischarbeit kann gar nicht hoch genug geschätzt werden. Julia war da gleicher Ansicht und so haben wir gemeinsam durch Joggen unsere inneren Schweinehunde überwunden. Danke und alles Gute für Lüneburg!

Danke sagen möchte ich auch meiner Freundin Patricia. Sie hat die emotionalen Wogen, die die Doktorarbeit aufgetürmt hat, immer wieder erfolgreich geglättet. Danke, dass es Dich gibt.

Faan harten toonke maad ik uk Mam, Aatj an min saster Wehna. Uun jonk an laacht stüünjen stun jam mi aleewen tu siidj. 



\section{CONTENTS}

1 GENERAL INTRODUCTION

1.1 Real-time MRI and Model-based Reconstruction . . . . . . . . . I

1.2 Motivation of Study .................. 2

1.3 Structure and Scope of Thesis . . . . . . . . . . . . . 3

1.4 Authorships and Individual Contributions ........... . 4

2 BASICS OF MAGNETIC RESONANCE IMAGING 5

2.1 Signal, Measurement, and Relaxation Processes . . . . . . . . . 5

2.2 Spatial Encoding and Image Reconstruction . . . . . . . . . . . 6

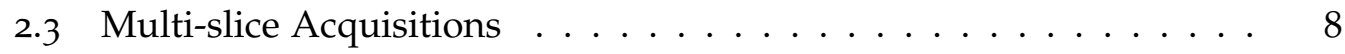

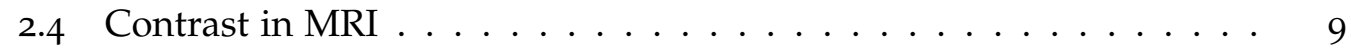

2.5 Steady-State Sequences . . . . . . . . . . . . . . . . 9

2.6 Quantitative MRI and $T_{1}$ mapping ............. . II

2.7 Adiabatic RF Pulses . . . . . . . . . . . . . . . . . . 12

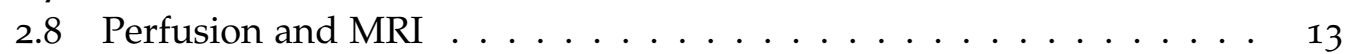

2.9 Pharmacokinetic Analyses .................. . 13

2.10 Contrast Agents in MRI . . . . . . . . . . . . . . . . 14

3 SPOILING WITHOUT ADDITIONAL GRADIENTS: RADIAL FLASH MRI WITH RANDOMIZED RADIOFREQUENCY PHASES

3.1 Introduction . . . . . . . . . . . . . . . 17

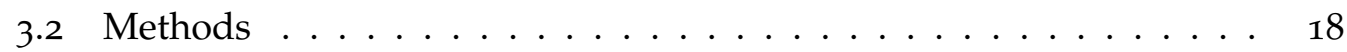

3.2.1 Spoiled FLASH Sequences .............. 18

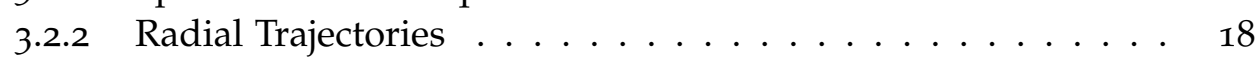

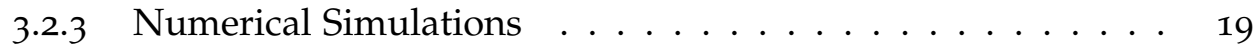

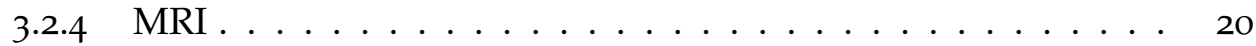

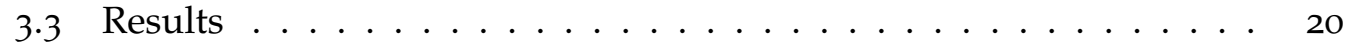

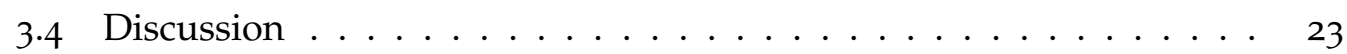

4 MULTI-SLICE T $_{1}$ MAPPING WITH SINGLE-SHOT INVERSION-RECOVERY $\begin{array}{ll}\text { FLASH } & 27\end{array}$

4.1 Introduction . . . . . . . . . . . . . . . . . 27

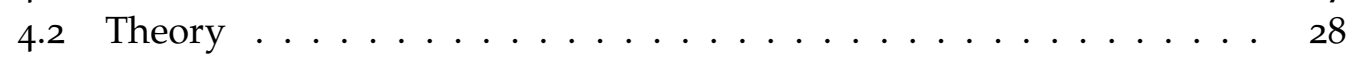

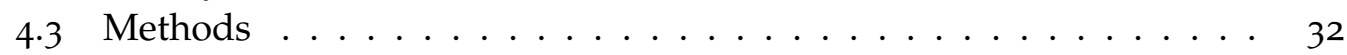

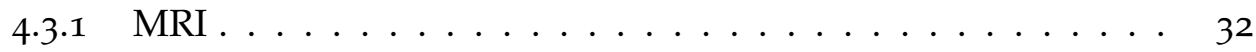

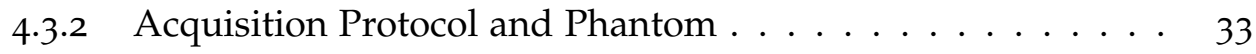

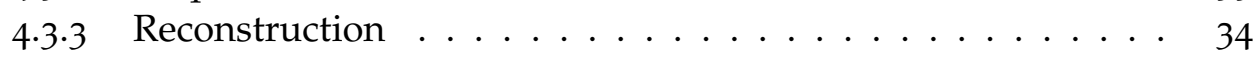


CONTENTS

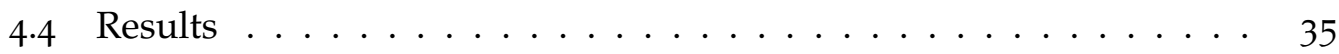

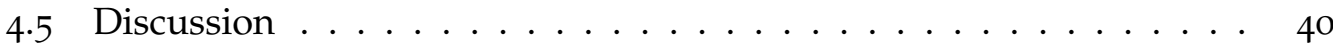

5 MODEL-BASED RECONSTRUCTION FOR $\mathrm{T}_{1}$ MAPPING USING SINGLESHOT INVERSION-RECOVERY RADIAL FLASH 43

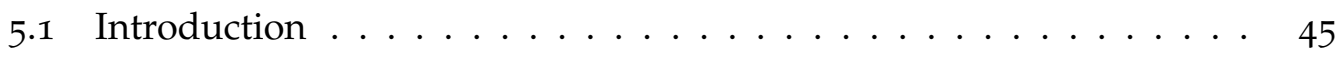

5.2 Methods ........................... 45

5.2.1 Signal Model and Optimization . . . . . . . . . . . . 45

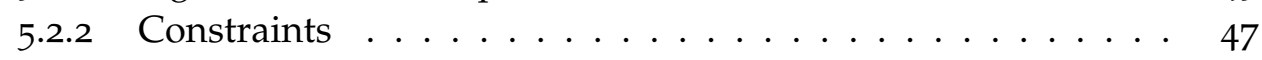

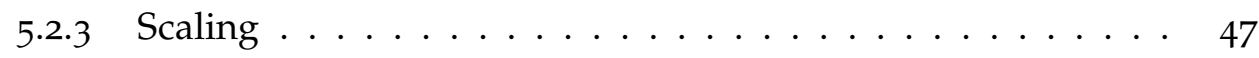

$5.2 .4 \quad$ MRI . . . . . . . . . . . . . . . . . 48

5.2 .5 Numerical Simulation . . . . . . . . . . . . . . . 49

5.2 .6 Preprocessing .................... 50

5.2 .7 Determination of Coil Sensitivity Profiles . . . . . . . . 50

5.3 Results ......................... 50

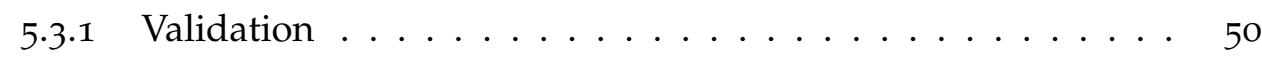

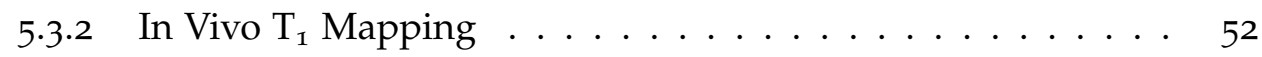

5.4 Discussion . . . . . . . . . . . . . . . . . 57

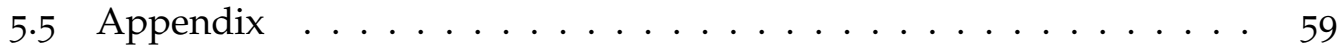

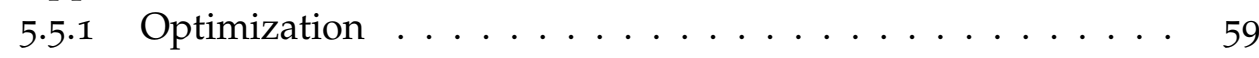

5.5 .2 Scaling ......................... 61

6 DYNAMIC T 1 MAPPING IN FIRST-PASS DCE-PERFUSION STUdIES 63

6.1 Introduction . . . . . . . . . . . . . . 63

6.2 Methods ............................. 64

6.2.1 Perfusion Phantom and Experimental Setup . . . . . . . . . . 64

6.2.2 Pharmacokinetic Model . . . . . . . . . . . . . . 65

6.2 .3 Contrast Agent . . . . . . . . . . . . . . . 68

6.2.4 Protocols and Reconstruction . . . . . . . . . . . 70

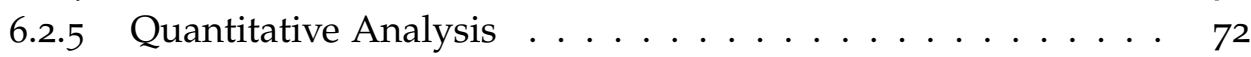

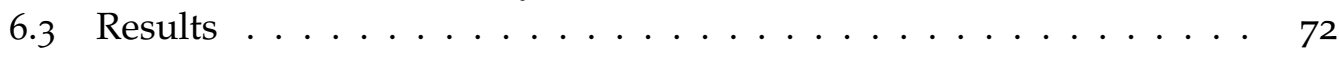

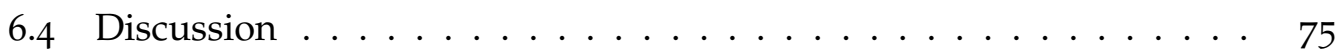

$\begin{array}{lll}7 & \text { SUMMARY } & 77\end{array}$

$\begin{array}{lc}\text { BIBLIOGRAPHY } & 81\end{array}$

$\begin{array}{ll}\text { ACRONYMS } & 93\end{array}$

xii 


\section{LIST OF FIGURES}

Figure 2.1 The fundamental dynamics in MRI: excitation, precession, and relaxation ................ 6

Figure 2.2 Spatial encoding in parallel imaging . . . . . . . . 7

Figure 2.3 Chemical structure of Gadobutrol . . . . . . . . . . . . 14

Figure 3.1 Numerical simulations of spoiling artifacts in radial gradientecho images .................... 21

Figure 3.2 Spoiling artifacts in gradient-echo images of a water-filled phantom ......................... 24

Figure 3.3 Spoiling artifacts in gradient-echo images of a transverse section through the human brain . . . . . . . . . . 25

Figure 4.1

Figure 4.2 Scheme of a three-slice inversion-recovery measurement . . 30

Figure $4 \cdot 3$ Plot of the derived analytical expression for a 3-slice inversionrecovery experiment . . . . . . . . . . 36 ROI-averaged signal time courses for all 6 compartments in the single-slice and 3 -slice measurement . . . . . . . . . .

Figure 4.4 Quantitative comparison between single-slice and multi-slice

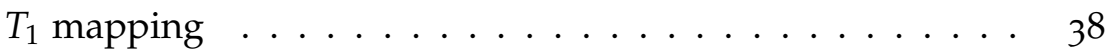

Figure 4.5 Reconstructed image series of the 5-slice experiment $\ldots \quad \ldots 38$

Figure $4.6 \quad T_{1}$ maps of a human brain (transverse sections) . . . . . . 39

Figure $5.1 \quad$ Model-based $T_{1}$ mapping of simulated data . . . . . . . $5^{1}$

Figure 5.2 Parameter maps and derived physical quantities $T_{1}$, spin density $M_{0}$, and flip angle $\alpha$ during iterative estimation . . . 53

Figure 5.3 Model-based $T_{1}$ mapping of a phantom with 6 compartments covering $T_{1}$ values from $0.3 \mathrm{~s}$ to $1.5 \mathrm{~s} \ldots \ldots . \ldots 54$

Figure 5.4 Model-based $T_{1}$ mapping of transverse section of the human brain and two abdominal sections . . . . . . . . . 55

Figure 5.5 Model-based $T_{1}$ mapping of a sagittal, coronal and transversal section of the human brain . . . . . . . . . . 56

Figure 6.1 Photographs of all parts in the first-pass perfusion experiment 66 Figure 6.2 Schematic setup of the in vitro first-pass perfusion experiment 67

Figure 6.3 Schematic illustration of the assumed contrast agent transfer in the tissue and in the phantom . . . . . . . . . . . 69

Figure 6.4 $T_{1}$ maps after model-based reconstruction exemplarily shown for 5 time points . . . . . . . . . . . . . . . 73

Figure 6.5 Longitudinal relaxation rates and calculated tracer concentrations as a function of time $\ldots \ldots \ldots . \ldots 74$ 


\section{LIST OF TABLES}

Table 4.1 Sequence parameters used in the multi-slice $T_{1}$ mapping ex-

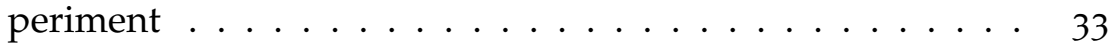

Table 5.1 Acquisition parameters for model-based $T_{1}$ mapping . . . . 49

Table 5.2 $\quad T_{1}$ relaxation times (single subject) in brain, liver and kidney 54

Table 6.1 Injection, sequence, and reconstruction protocols used in the perfusion experiment . . . . . . . . . . $7^{1}$

Table 6.2 Quantitative results of the perfusion experiment $\ldots \ldots .75$ 


\section{GENERAL INTRODUCTION}

\section{I REAL-TIME MRI AND MODEL-BASED RECONSTRUCTION}

Magnetic resonance imaging (MRI) is a non-invasive imaging technique that produces three-dimensional representations of the interior of a body without harmful radiation. These images are used for clinical diagnoses and medical interventions routinely all over the world. However, when compared to other imaging modalities such as X-ray radiography or ultrasound, MRI is a slow technique. The underlying physical principles reveal where the inherent speed bottleneck is located: The raw data necessary to form an image is acquired in a multitude of independent excite-and-sample experiments. These repetitive measurements have been tremendously sped up in the past decades by technical innovations such as fast sequences (e.g., fast low-angle shot (FLASH) [1, 2] or rapid acquisition with refocused echoes (RARE) [3]), strong gradient-amplifier systems, and the use of multiple receiver coils. Nowadays, physical and physiological constraints (relaxation times, peripheral nerve stimulation, energy deposition, etc.) limit the speed of data acquisition fundamentally. While speed is not the ultimate criterion in many applications, a fast and continuous stream of magnetic resonance (MR) images is required when studying functional processes like speaking, swallowing, or the beating heart. The timescale on which these processes take place directly dictate the frame rates that are necessary to achieve. MRI techniques that deal with such high frame rates are commonly referred to as real-time MRI, although this term has been used in the literature inconsistently with respect to different temporal and spatial resolutions.

In the last ten years, our group developed real-time imaging methods that rely on radially encoded gradient-echo sequences with minimal repetition time (TR) [4]. These so-called steady-state sequences produce a continuous stream of raw data, a major prerequisite for real-time imaging. Image reconstruction algorithms that make explicit use of parallel imaging, i.e., the use of multiple receiver coils for signal readout, were successfully combined with the stream of radial readouts [5]. These advanced algorithms formulate the task of image reconstruction as a nonlinear inverse problem, solve it iteratively and also allow to include prior knowledge into the image formation process, for instance in form of redundancies inherent to time series. By these means, the amount of raw data needed for reconstruction of a single image out of a time series was further reduced, enabled imaging in real time with 50 frames per second and beyond [6-8], and made a variety of physiological studies possible [9-16]. 
The use of data redundancies can also be found in MRI applications not related to image time series. Quantitative imaging methods aim for an absolute quantification of specific parameters such as $T_{1}$ or $T_{2}$ relaxation times, temperature, macromolecular content, diffusivity, or magnetic susceptibility. In most cases, a certain MR signal is produced that can be modeled as a function of a few unknowns, among them the parameter of interest. In conventional parameter mapping methods a set of contrasts is prepared, independently reconstructed as a set of images, and finally fitted by a signal model in a pixel-by-pixel fashion. While straightforward to perform, such 2-step quantification methods that separate image reconstruction and quantification make only suboptimal use of the available raw data. The fact that the reconstructed set of images can be fully characterized by a few parameter maps already indicates a high degree of redundancy. Model-based reconstruction techniques make explicit use of this evidence by directly reconstructing parameter maps from the measured raw data. Although this approach comes at the cost of increased complexity and computational demands, it has been adapted and successfully applied to different quantitative applications [17-23].

\subsection{MOTIVATION OF STUDY}

Quantitative assessment of tissue perfusion by means of contrast agent (CA) injection is called dynamic contrast-enhanced (DCE) MRI. In this technique, a bolus of CA is rapidly injected into the blood stream and travels to the tissue of interest where its first pass creates intensity changes in the tracked MR signal. Knowledge about concentration distributions in the bolus together with its induced tissue response can be transformed into quantitative maps of perfusion parameters such as capillary blood flow, relative blood volume, and mean bolus transit time. The connection of dynamically changing signal intensities together with the requirement for a sufficient spatial resolution render the application of real-time imaging ideas to first-pass perfusion experiments promising.

On the other hand, relaxation times play an import role in all DCE MRI methods because changes in CA concentration can only be detected indirectly by altered relaxation times leading to signal intensity changes. This dependence establishes a strong link between DCE MRI and quantitative $T_{1}$ mapping. The latter is often realized by techniques that exhibit redundancies in their respective data acquisition and seem therefore a good candidate for a model-based reconstruction approach.

Consequently, this thesis concentrates on methodological developments in both acquisition and reconstruction techniques when applying concepts from real-time imaging to the field of DCE MRI. 


\subsection{STRUCTURE AND SCOPE OF THESIS}

In this thesis, four different aspects of quantitative MRI techniques are addressed and presented in independent chapters.

Chapter 3 deals with image artifacts occurring in radially sampled FLASH images. Residual steady-state transverse magnetizations are identified as the source for parasitic concentric rings and their dependencies on the employed sampling pattern are investigated. Randomized radiofrequency (RF) phases are proposed to effectively suppress these undesired coherences without the need for time-costly spoiler gradients. The resulting spoiling performance is assessed in numerical simulations, phantom experiments and in vivo MRI studies of the human brain. The developed spoiling scheme is implemented on our clinical MRI scanner together with k-space sampling schemes favorable for model-based reconstructions and hence forms a major building block for all studies presented here. As the findings and the proposed solution are of general interest and particularly relevant for highspeed real-time MRI, I published this work in the peer-reviewed journal "Magnetic Resonance in Medicine". Consequently, chapter 3 is a reprint of the original article.

In chapter 4, a signal model is developed that describes the magnetization time course in a multi-slice single-shot inversion-recovery FLASH experiment. For this purpose, the entire relaxation process is partitioned into regimes in which the respective dynamics are governed by mono-exponential relaxation. Interleaved application of time evolution operators finally yields an analytical expression that extends the expression for single-slice $T_{1}$ mapping [24] towards multi-slice imaging. The validity of this model has been verified in simulation and phantom experiments. In [25], a co-authored publication of my colleague, this model was used for multi-slice $T_{1}$ mapping of human brain and abdominal sections. At this stage, quantification of $T_{1}$ was performed by pixelwise fitting of the derived signal model to a time series of reconstructed images (2-step method).

The segregation of reconstruction and quantification was removed in the modelbased $T_{1}$ mapping method presented in chapter 5 . Here, a joint reconstruction of the $T_{1}$, spin-density, and flip-angle map directly from the raw data was formulated as a nonlinear inverse problem and solved by the iteratively regularized Gauss-Newton method (IRGNM). Prior knowledge was included into the reconstruction routine by reparametrization of the original signal model and introduction of parameter-specific regularization terms. A sampling trajectory based on the Golden Angle scheme [26] in combination with the novel RF spoiling scheme allowed for fast data acquisition, and the outsourcing of parallelizable code modules to the graphics processing unit (GPU) ensured fast reconstruction. Problems such as scaling of unknowns, bound-constraint optimization, binning size determination, and suppression of noise from outer k-space regions are addressed and effective solutions presented. This comprehensive work forms a stand-alone method and 
was submitted to "NMR in Biomedicine" (in revision at time of thesis submission). Chapter 5 is a reprint of the respective manuscript without modifications.

This novel technique for fast and accurate high-resolution $T_{1}$ mapping finds its direct application in first-pass perfusion experiments performed in vitro (chapter 6). In these experiments, a commercial DCE MRI phantom was employed to mimic perfusion similar to in vivo situations with full control over the involved exchange rates. An in vitro flow cycle for monitoring MR signal changes due to the presence of CA was set up and the individual parts involved are depicted and described in function. This experimental setup offers a huge variety of possible simulation scenarios and can for example be used to evaluate future acquisition protocols and reconstruction algorithms in the context of quantitative DCE perfusion. The specific combination of injection and reconstruction protocol presented in chapter 6 is an exemplary choice and demonstrates general feasibility.

Individual findings, discussed in detail separately in each chapter, are summarized in chapter 7 . This also includes an outlook on future work.

\subsection{AUTHORSHIPS AND INDIVIDUAL CONTRIBUTIONS}

Of course, research in a working environment as established at our institute implies collaboration and joint projects. To clarify authorships and individual contributions, each chapter contains a preface explicitly stating how co-authors and collaborators contributed to the respective work. 


\subsection{SIGNAL, MEASUREMENT, AND RELAXATION PROCESSES}

Atomic nuclei with an odd number of either or both protons and neutrons carry an intrinsic angular momentum, the nuclear spin. This quantum mechanical entity is connected to a nuclear magnetic moment that every nucleus with nonzero spin posses. Similar to a macroscopic magnet, where the classical magnetic moment determines the torque it experiences in an external magnetic field, the nuclear magnetic moment interacts with an external magnetic field and leads to a system whose energy depends on the orientation of the magnetic moment with respect to the axis of the external field. The orientation parallel to the external field is energetically preferred over an anti-parallel orientation, similar to a compass needle in the earth's magnetic field. The resulting energy difference is proportional to the strength of the external magnetic field and explains to a large degree the relentless pursuit for MR scanners with higher field strengths. However, even large ensemble of spins - as present in tissue - only result in a small net magnetic moment due to thermal fluctuations. For in vivo situations, these fluctuations are characterized by the body temperature and hence constitute an unalterable condition in MRI. This small net magnetic moment is the origin for the low sensitivity that all MR techniques suffer from and that has to be compensated by long scan times, large voxel sizes, and expensive equipment.

If a large ensemble of nuclear spins is suddenly exposed to a strong magnetic field, a new thermal equilibrium will eventually build up that respects the orientation-dependent energy differences of the nuclear magnetic moments in the sample. This build up process does not take place instantaneously but was found to show an exponential transition behaviour that could be quantified by an exponential time constant $T_{1}$, known as the longitudinal relaxation time (or historically spin-lattice relaxation time). Theoretical considerations connect the heuristically introduced $T_{1}$ relaxation time to microscopic fluctuations caused by the tumbling motion of molecules. These small motions lead to local magnetic field disturbances that, in turn, induce transitions between energy states of the spin system. From this viewpoint it is evident why $T_{1}$ relaxation times not only depend on the nucleus but also on temperature, viscosity, and microstructure of the sample.

The quantum nature of a nuclear magnetic moment - in contrast to a classic compass needle - leads to another motion, namely the precession of the spin's polarization around the static magnetic field. A full quantum mechanical treatment 

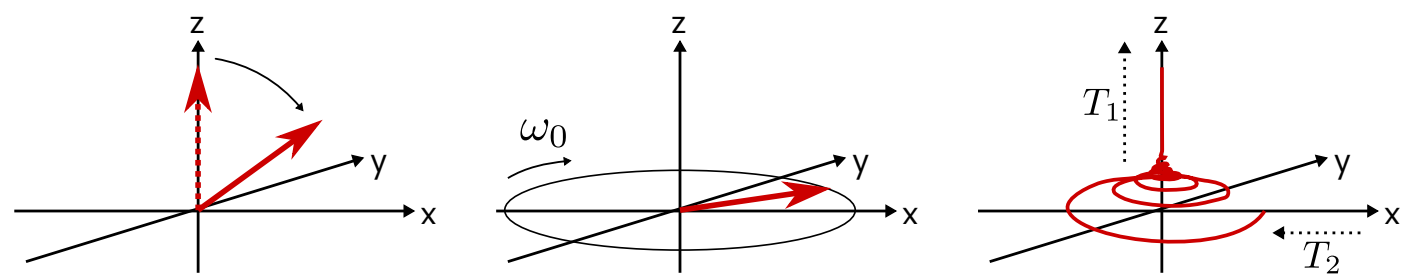

Figure 2.1: THE FUNDAMENTAL DYNAMICS IN MRI: EXCITATION, PRECESSION, AND RELAXATION. By application of a RF pulse the macroscopic magnetization can be tipped away from its equilibrium state (left), the magnetization precesses then about the axis of the static magnetic field (middle), and longitudinal and transverse relaxation processes finally lead to a recovery toward the equilibrium state (right).

(as found in [27] or [28]) reveals that the polarization precesses with the Larmorfrequency $\omega_{0}$, which is connected to the gyromagnetic moment $\gamma$ of the nucleus and the field strength $B_{0}$ by

$$
\omega_{0}=-\gamma B_{0} .
$$

For protons at $B_{0}=3 \mathrm{~T}$ the magnitude of the Larmor-frequency amounts to approximately $128 \mathrm{MHz}$. This precession is used in both nuclear MR spectroscopy and imaging to measure the macroscopic magnetization. For this purpose, the magnetization initially aligned in direction of the external magnetic field is rotated into the transverse plane by applying a RF pulse. The precession motion governing the dynamics of each individual spin polarization also leads to a precessing transverse net magnetization (see fig. 2.1), which induces a voltage in the readout coils: the actual MRI signal. However, the excited transverse magnetization is not only affected by longitudinal relaxation but also by a decay process called transverse relaxation. Similar to the effects causing $T_{1}$ relaxation, also small field fluctuations on the microscopic scale make it impossible to keep perfect synchrony between the precessing spins. This irreversible dephasing process causes a decay of the transverse net magnetization and is characterized - in close analogy to $T_{1}$ relaxation - by the exponential time constant $T_{2}$, called the transverse or spin-spin relaxation time constant.

In a MR scanner, this dephasing process is even accelerated as technical imperfections on the one hand and magnetic susceptibility effects on the other hand lead to inhomogeneities in the static magnetic field. To take these additional sources of dephasing into account, an effective or observed transverse relaxation time is often introduced and denoted by $T_{2}^{*}$.

\subsection{SPATIAL ENCODING AND IMAGE RECONSTRUCTION}

Spatial encoding in a state-of-the-art MRI system is realized by two complementary principles: Gradients applied before and during signal readout exploit the spatial 


\section{Object}

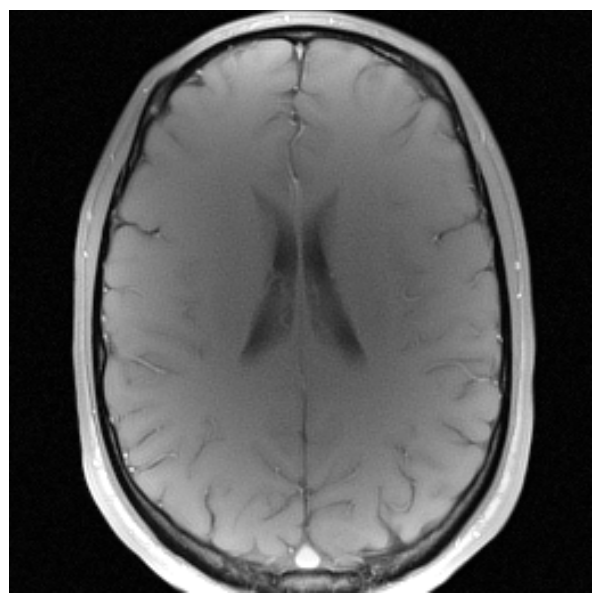

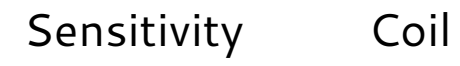

Profile Image

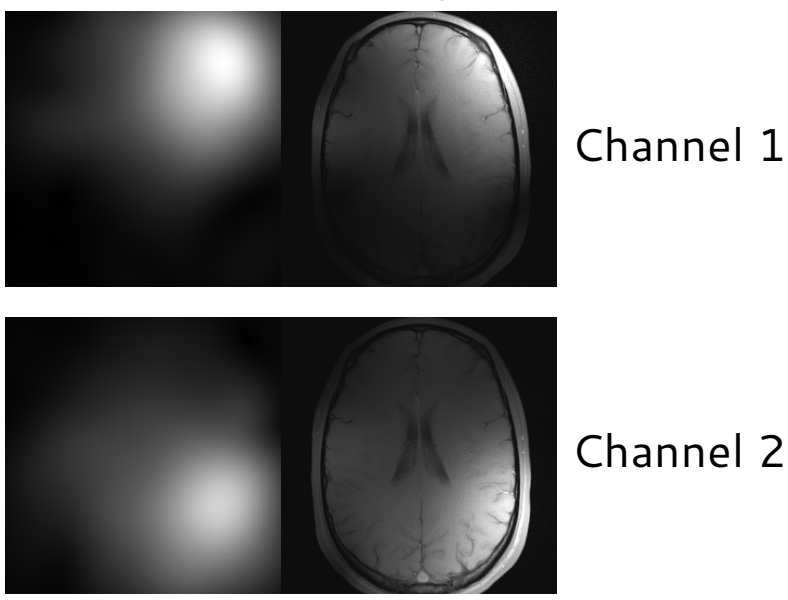

Figure 2.2: SPATIAL ENCODING IN PARALLEL IMAGING. In parallel imaging spatial encoding is realized through differences in coil sensitivity profiles. Simultaneous signal acquisition with multiple readout channels provides a set of individual coil images. Each coil image (right) reflects a weighted version of the underlying common object (left) according to the individual coil sensitivity profile (middle).

dependency of the Larmor-frequency and the simultaneous signal detection with multiple receive coils utilizes differences in the receive coil sensitivities for spatial discrimination (see fig. 2.2). The former gradient encoding is established since the foundation of MRI in the 1970s [29] whereas the latter technique of parallel imaging was introduced in the late 1990s and is still subject of ongoing research on both hardware and reconstruction side. Combining both spatial encoding principles results in MR experiments that can be described by a basic signal equation that relates the magnetization distribution $M_{\perp}(\vec{x})$ to the observed time signal $s_{j}(t)$ in the $j$-th receive coil:

$$
s_{j}(t)=\int_{V} M_{\perp}(\vec{x}) c_{j}(\vec{x}) e^{-2 \pi \vec{k}(t) \vec{x}} \mathrm{~d} \vec{x},
$$

where $c_{j}(\vec{x})$ represents the (complex-valued) sensitivity profile of the $j$-th receive coil, $V$ the support of the object, and $\vec{k}(t)=\frac{\gamma}{2 \pi} \int_{0}^{t} \gamma \vec{G}\left(t^{\prime}\right) \mathrm{d} t^{\prime}$ the spatial frequencies that are sampled according to the chosen gradient evolution $\vec{G}(t)$.

This equation states the measured MR signal is the Fourier transform of the magnetization distribution weighted by the individual coil sensitivity profiles. The Fourier transform domain in MRI is known as k-space and the path on which the vector $\vec{k}(t)$ traverses this space is called k-space trajectory or sampling pattern.

A fully sampled k-space that has been sampled on a Cartesian grid can be transformed into an image by simply performing a fast Fourier transform (FFT) on the 
raw data matrix. This image reconstruction method was the standard technique for more than 20 years due to its simplicity, speed, and effectiveness.

Since arbitrary sampling patterns are possible, also non-Cartesian trajectories can easily be realized, each with their individual advantages and disadvantages. From these non-Cartesian patterns, the most popular are radial and spiral trajectories. Image reconstruction of these type of data acquisitions typically include a step called gridding or regridding describing the process of interpolating non-Cartesian samples to a Cartesian grid so that the FFT algorithm can be applied again. However, when only undersampled data is available or when physical effects that not fit into the Fourier framework have to be incorporated into the reconstruction, iterative algorithms in connection with appropriate models can improve image quality at the cost of increased computational demands. The need for iterative algorithms arises here from the fact that the involved problems are typically too large to be solved in one explicit step or that nonlinearities preclude explicit inversion of the corresponding equations.

The simplest examples for such iterative methods are found in sensitivity encoding (SENSE) algorithms that estimate the desired image iteratively from measured raw data and known coil sensitivity profiles [30]. The inclusion of the coil profiles into the vector of unknowns yields a bilinear parallel imaging problem that can be solved iteratively [31] and explicit modelling of physical effects such as $T_{1}, T_{1}^{*}$, and $T_{2}$ relaxation [18-21, 32, 33], field inhomogeneities [34], diffusion [22], flow [23], and others opens the field of model-based reconstruction.

\subsection{MULTI-SLICE ACQUISITIONS}

Slice selection in MRI exploits the fact that the application of gradient fields lead to spatially dependent Larmor-frequencies. As excitation by RF radiation requires the corresponding RF frequency to match the Larmor-frequency, gradients immediately provide a tool to select which region of the three-dimensional volume to excite. In general, MR sequences always employ a combination of gradient fields and spatially selective RF pulses. A finite support of the frequencies contained in a transmitted pulse ensures - in combination with the gradient fields - spatial selectivity. For two-dimensional imaging, the excitation of only a small slice of the entire body is desired. For this purpose the bandwidth of the excitation pulses is chosen narrow. The direct correspondence of frequency and spatial distance along the gradient's direction leads to the option of tuning the frequency of the RF pulse in such a way that a slice at a certain position is excited. Multislice imaging experiments cover a three-dimensional region by dividing it into a set of individually acquired slices. However, this set of slices is typically not acquired by performing a series of single-slice measurements but rather by exciting multiple slices within each TR interval. For this purpose the same slice selection gradient is used several 
times in combination with different RF pulse frequencies according to the desired positions of the slices. Due to the fact that imperfect RF pulses also partly excite spins in the immediate neighbourhood of an excited slice, special care has to be taken in covering a volume with two-dimensional slices. To avoid this undesired "slice crosstalk" typically a certain gap is left between individual slices (resulting in a non-contiguous volume coverage) or the slice excitation order is designed in such a way that first only even-numbered and then only odd-numbered slices are excited. The latter strategy gives the unintendedly excited magnetization enough time to recover toward equilibrium before the particular slice itself is excited.

\subsection{CONTRAST IN MRI}

In contrast to computed tomography (CT) images, in which each pixel reflects the mean attenuation coefficient of the tissue (relative radiodensity), MR images usually show signal intensities that are functions of multiple parameters. Some of these parameters are intrinsic as they depend on the imaged tissue (relaxation times $T_{1}$ and $T_{2}$, proton density, diffusion times, flow rates, etc.), while others are related to the parameters of the MR sequence (flip angle, bandwidth, etc.) and to the chosen timings (repetition time, echo time, etc.). By choosing a specific combination of sequence parameters it is possible to obtain images that are strongly depending on particular intrinsic parameters while the influence of others is mainly eliminated. For instance, by choosing both, a short echo time (TE) and a short TR in spin echo imaging, the influence of the $T_{2}$ relaxation time on the signal intensity can be mainly suppressed and images are obtained that reflect pixel-wise differences in the $T_{1}$ relaxation time. The property of an image to depend on certain tissue specific parameters more than others is called image contrast and by changing sequence and timing parameters MR images with different contrasts can be obtained. As pathologies do not affect all tissue parameters equally well, clinical MR routines typically comprise several image acquisitions with different contrasts.

However, these resulting images still exhibit signal intensities that depend on multiple parameters and do not quantify individual parameters. The intensity of the spin echo signal, for example, is always proportional to the proton density (water content) of each pixel independent of the chosen contrast.

\subsection{STEADY-STATE SEQUENCES}

Steady-state sequences are gradient echo sequences with short TR that satisfy the relation TR $\ll T_{2}, T_{1}$. The time period between two successive excitation pulses is so short that neither full $T_{1}$ nor full $T_{2}$ relaxation takes place. As a consequence, neither the longitudinal nor the transverse magnetization components fully relax, both magnetization types are affected by the next excitation pulse, and multiple 
spin echoes and stimulated echoes contribute to the measured signal intensities. Under certain conditions, these contributions add up coherently, the obtained signal intensities stay constant from TR to TR, and a dynamic equilibrium, the so-called steady-state is reached. The intensity of this steady-state in principle depends on both, the relaxation time $T_{1}$ and $T_{2}$, where the latter determines the lifetime of transverse components within the pulse train. These transverse components determine the obtained image contrast and can be manipulated according to the desired contrast. To remove the steady-state's sensitivity to the $T_{2}$ relaxation time, it is necessary to minimize the contributions of the transverse components to the signal. This strategy is called "spoiling" and has commonly been implemented in two ways: In so-called gradient spoiling techniques, strong gradients at the end of each TR interval are used to dephase residual transverse components [35, 36]. However, this spoiling strategy suffers from some limitations. First of all, the spatial dependency of the gradients leads to pixel-dependent phase evolutions, which is adverse as the spoiling will be spatially non-uniform. The second limitation lies in the fact that magnetization cannot be destroyed on the time scales smaller than the relaxation time $T_{2}$. The strong gradients only dephase the magnetization in the sense of distributing the individual transverse magnetization components so that at the end of one TR the net transverse magnetization averages to zero. However, subsequent RF pulses may invert the phases of transverse magnetization components and the gradient event - intended for dephasing - in this case will act as a full rephasing gradient. To avoid these detrimental situations the strength of the spoiler gradients is typically varied in a heuristic manner that includes all gradient axes.

An alternative technique that does not suffer from the limitations of gradient spoiling techniques is based on the manipulation of the RF-transmitter phase and has nowadays been adapted by all major MR vendors as the de facto spoiling standard. RF spoiling employs constant, non vanishing zero order gradient moments from TR to TR and uses instead the phase of the RF-transmitter system to create incoherent contributions from transverse components. It is superior to gradient spoiling methods in theory and praxis due to its spatially invariant spoiling effectiveness and because an analytical derivation of the optimal phase variation scheme could be found [37].

A perfectly spoiled steady-state signal only contains contributions from the longitudinal magnetization present prior to the excitation pulse and is hence not influenced by the relaxation time $T_{2}$. This leads to purely $T_{1}$-weighted images. An opposing strategy is to guaranty a coherent contribution of all transverse components to the steady-state signal. In this case the resulting images will benefit from an increased signal intensity and will exhibit a mixed contrast which is commonly denoted as $T_{2} / T_{1}$ weighting. The prerequisites for the formation of such a steady-state are vanishing zero order gradient moments in each TR interval. This condition assures that the gradients have no effect on the steady state and the re- 
sulting magnetization dynamics of such a sequence is called balanced steady-state free precession (bSSFP). With an alternating RF phase of the excitation pulses the bSSFP-sequence results in the highest signal strength of all steady-state sequences, but is rather sensitive to off-resonance effects that interfere strongly with the zero phase condition.

\subsection{QUANTITATIVE MRI AND $T_{1}$ MAPPING}

Quantitative MRI provides quantitative information about tissue in contrast to conventional MRI where images exhibit a certain contrast, i.e., are more sensitive to specific tissue parameters than to others, but do not strictly quantify these parameters. Quantitative imaging methods typically yield parameter maps that quantify a certain parameter pixel-wise. Generally, these methods have gained interest during the past decades as quantitative parameter maps allow a comparison of results across subjects and are independent of the MR hardware or sequences used. Conventional parameter mapping techniques typically prepare a series of contrasts, reconstruct the corresponding images, and obtain quantitative information in a post-processing step in which a certain model is fitted pixel-wise to the images. Parameters that are accessible in this fashion include the relaxation times $T_{1}, T_{2}$ and $T_{2}^{*}$, the proton density, flow velocities, the apparent diffusion coefficient or diffusion tensor information, tissue temperature, blood flow, magnetization transfer ratios, metabolite concentration, and others.

The gold standard method for $T_{1}$ mapping is based on NMR experiments performed more than sixty years ago $[38,39]$. The method is nowadays known as inversion recovery (IR) $T_{1}$ mapping and still relies on inversion of the longitudinal magnetization and sampling the MR signal as the magnetization recovers toward thermal equilibrium. The underlying sequence consists of two RF pulses separated by the variable inversion time (TI). The initial magnetization is inverted by the first pulse, recovers for the time period TI, and gets tipped into the transverse plane by the second pulse. The speed bottleneck of this method lies in the fact that each inversion requires a prior recovery of longitudinal magnetization, which can only be achieved by a correspondingly long repetition time. This drawback is exacerbated by the repetitive nature of each MR imaging process: In its generic version, each TR interval of the IR sequence yields only one portion of raw data. As $T_{1}$ mapping requires a multitude of images acquired at different inversion times, the IR $T_{1}$ mapping method is - though accurate and robust - too time-consuming for mapping in clinically feasible times. Two alternative techniques are commonly employed that do not suffer from long acquisition times. The so-called variable flip angle method acquires two or more spoiled gradient-echo images that differ only in the nominal flip angle used. Information about $T_{1}$ is then extracted from the different steadystate signal intensities. This method is capable of three-dimensional $T_{1}$ mapping 
in clinical feasible times but requires exact knowledge about the actual flip angle and assumes a perfectly spoiled steady state in a sense of complete absence of any transversal coherences. Both prerequisites are hard to meet and lead to a variety of sequence variants and extensions that try to mitigate resulting problems. The second alternative method, the Look-Locker (LL) sequence is closely related to the IR sequence. Here, the magnetization is also prepared with an initial inversion pulse, but instead of acquiring a single sample of the recovery curve per TR, a train of equally spaced, low flip angle pulses generates a large number of gradient echoes. As the relaxation process is interleaved by repetitive excitation, the observed signal does not reflect the pure $T_{1}$ recovery but also exhibits a contribution from the low flip excitations. To separate these two effects, knowledge about the actual flip angle is required, however, in contrast to the variable flip angle method, the measured steady state can be used to obtain this additional information. Also here perfect spoiling is assumed when fitting the measured signal time courses.

\subsection{ADIABATIC RF PULSES}

Excitation pulses in MRI sequences commonly consist of short events during which the amplitude of the RF magnetic field is varied while keeping the transmit frequency constant at the Larmor-frequency of the system. For this on-resonant excitation, the time integral of the field amplitude is proportional to the realized angle between the initial and final magnetization vector. This angle is commonly referred to as the flip angle. The degree to which a homogeneous excitation over the entire imaged object can be realized, depends primarily on the spatial homogeneity of the RF magnetic field. For certain coil geometries and especially static field strengths of $3 \mathrm{~T}$ and beyond, strong spatial variations of the field are observed. This non-uniform excitation leads to several problems including image shading, incomplete fat suppression, and reduced signal-noise ratio (SNR). In the context of $T_{1}$ mapping, this non-uniform excitation is particularly mitigating the efficiency of inversion pulses. Any deviation of the intended $180^{\circ}$ translates into a reduced magnetization after inversion. Depending on the model used, this lack of efficiency can translate into severe underestimation of the true $T_{1}$ values. To overcome this problem, special RF pulses can be used that do not obey the direct relationship between amplitude and realized flip angle. When both, magnetic field amplitude and frequency are varied during application of the pulse, modulation schemes can be found that realize nominal flip angles precisely even if the actual field amplitudes deviate from the nominal by a constant factor. The condition for this robustness is solemly a sufficiently strong RF magnetic field. Such pulses are called adiabatic pulses and are particularly hard to design in the presence of off-resonances. These off-resonances, however, are naturally arising when considering adiabatic pulses 
for slice selection. Adiabatic inversion pulses in $T_{1}$ mapping applications are therefore typically non-selective, i.e. they invert the magnetization in an entire volume.

\subsection{PERFUSION AND MRI}

Perfusion is the physiological process of a body to deliver blood to organs or parts of organs. Perfusion ensures supply of the tissue with sufficient oxygen and nutrients as well as removal of metabolites and carbon dioxide. Quantification of perfusion can be obtained by nuclear medicine methods such as positron emission tomography (PET) or single photon emission tomography (SPECT) but also with imaging modalities such as CT and MRI. The two different methods in MRI for quantitative perfusion measurements that have established so far are arterial spin labeling (ASL) techniques and MRI techniques that rely on exogenous contrast agents. The latter are further divided into dynamic contrast-enhanced (DCE) and dynamic susceptibility contrast-enhanced (DSC) MRI techniques. ASL uses the arterial blood itself as an endogenous CA by tagging it in a magnetization preparation step whereas in both DCE and DSC MRI a CA is injected intravenously to change the magnetic susceptibility of the blood and to generate a change in MR signal strength depending on the concentration of CA. DSC MRI monitors the passage of a CA bolus by a series of $T_{2}$ or $T_{2}^{*}$ weighted images and is mainly used in the brain to access the cerebral blood volume whereas DCE MRI is based on the acquisition of a series of $T_{1}$-weighted images and is used in a variety of clinical perfusion imaging applications involving different organs. The use of an exogenous CA allows DSC and DCE perfusion methods to achieve a substantially higher SNR when compared to ASL techniques.

The physical principles behind DSC and DCE measurements are relatively simple: A high concentration CA bolus is injected intravenously and travels with the blood stream to the tissue of interest where it induces signal intensity changes. These changes are continuously monitored and finally yield signal time courses that can be converted into concentration time courses. Quantitative parameter maps are then generated in a post-processing step that involves pharmacokinetic modelling of the physiological exchange processes. A clinician can finally obtain spatial information about potential perfusion deficits from these maps.

\subsection{PHARMACOKINETIC ANALYSES}

Given the measured concentration time courses, pharmacokinetic modeling in DCE MRI provides information about how the CA is distributed in the perfused tissue. Most models rely on the concept of a compartments, i.e., they describe exchange processes by mathematical entities representing interacting components. The administered CA is assumed to diffuse to and from these compartments where each 
compartment is fully characterized by its (homogeneous) CA concentration. Rate equations are then exploited to derive a set of differential equations that govern the exchange of CA. These theoretical models can finally be fitted to the measured data to obtain quantitative parameters in terms of involved exchange rates and relative compartment sizes.

\subsection{CONTRAST AGENTS IN MRI}

Contrast agents as used in clinical routine are typically gadolinium (Gd) based complexes. The metal Gd belongs to the lanthanoid series of elements and forms trivalent ions. Gadolinium(III) ions are toxic to humans and other mammals and can only be administered in combination with chelates that form strong bonds with the ions to ensure a safe excretion. With seven unpaired $4 \mathrm{f}$ suborbital electrons each Gd ion carries a large spin electron moment that interacts with the nuclear magnetic moments of adjacent water protons through dipole-dipole interactions. This effect shortens the $T_{1}$ relaxation time constant of the water protons by a factor that is proportional to the number of Gd ions around, which is given by the concentration of CA. However, magnetic field gradients in the vicinity of the paramagnetic Gd chelates also lead to an increased dephasing of transverse coherences, which results in shorting of the $T_{2}$ (or $T_{2}^{*}$ ) relaxation times of referring protons. These two effects are commonly known as longitudinal and transverse relaxivity of the CA and quantified in units of $\mathrm{L} / \mathrm{mmol} / \mathrm{s}$.

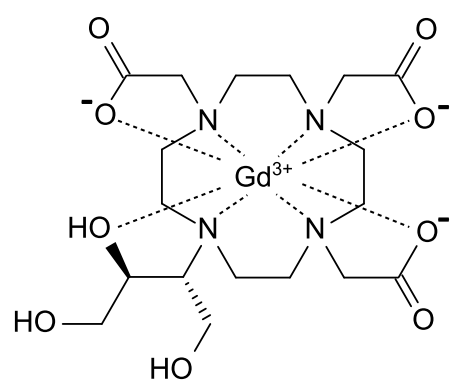

Figure 2.3: Chemical structure of Gadobutrol. From: Wikimedia Commons, Anypodetos, CC0 1.0

The paramagnetic properties of Gd based contrast agents are used to improve the visibility of anatomical structures in the body and, in dynamic imaging, to translate changes in CA concentration to changes in signal intensity as measured in a series of $T_{1}$ or $T_{2}^{*}$ weighted images. Figure 2.3 shows the chemical structure of Gadobutrol, a Gd-based CA that was used in this thesis for the perfusion studies. Gadobutrol is a non-ionic complex consisting of the Gd(III) ion and a macrocyclic ligand. 
SPOILING WITHOUT ADDITIONAL GRADIENTS: RADIAL FLASH MRI WITH RANDOMIZED RADIOFREQUENCY PHASES

The following is a reprint of the original article

V. Roeloffs, D. Voit, and J. Frahm. "Spoiling without additional gradients: Radial FLASH MRI with randomized radiofrequency phases". Magnetic Resonance in Medicine (June 2015). DoI: 10.1002/mrm. 25809.

$V R$ implemented the simulations, designed and performed the experiments, and analyzed the results. $V R$ and $D V$ implemented the sequence variants and the sampling trajectories. $V R$ and $J F$ wrote the manuscript. 


\section{ABSTRACT}

PURPose: To develop a method for spoiling transverse magnetizations without additional gradients in order to minimize repetition times for radial FLASH MRI.

Methods: Residual steady-state transverse magnetizations and corresponding image artifacts were analyzed for radial gradient-echo sequences with constant and randomized RF phases in comparison to a sequence with refocused frequency-encoding gradients, constant spoiler gradient, and conventional RF spoiling (gold standard). The spoiling performance was assessed for different radial trajectories using numerical simulations, phantom experiments and in vivo MRI studies of the human brain.

RESULTS: Simulations as well as phantom and in vivo measurements reveal a highly efficient spoiling capacity for randomized RF phases and radial FLASH sequences without the need for gradient rewinding and spoiler gradients. The data also demonstrate a strong dependence of the spoiling performance on the chosen radial trajectory, i.e., the azimuthal angular increment between successive projections, with excellent results for an interleaved multi-turn scheme.

CONCLUSION: Effective spoiling of transverse magnetizations in radial FLASH MRI may be achieved by randomized RF phases without additional spoiler gradients. The technique allows for short repetition times as required for high-speed real-time MRI. 


\subsection{INTRODUCTION}

Since its inception the development of MRI has largely been driven by the quest for speed with the ultimate demand defined by recent advances in real-time MRI, e.g., see $[6,8]$. The underlying concept for serial imaging relies on steady state gradient-echo sequences that either include or exclude transverse magnetizations to yield $T_{2} / T_{1}$ or $T_{1}$ contrast. Respective techniques are usually referred to as (fully balanced) steady-state free precession (SSFP), refocused FLASH or spoiled FLASH. Because the former two require extended gradient waveforms for spin rephasing in each repetition interval TR to achieve a zero or constant net phase, respectively, the minimum TR value is constrained by the extra gradient timing. As a consequence, the shortest possible TR for high-speed real-time MRI may be obtained by using $T_{1}$ or spin-density contrast in conjunction with technical means that minimize the time for eliminating transverse coherences.

Early attempts to $T_{1}$-weighted gradient-echo MRI entirely relied on gradient spoilers which prolong the TR interval and for real-time MRI directly translate into decreased frame rates. In principle, this problem may be overcome by RF spoiling $[37,41]$ which varies the common phase for RF excitation and signal reception from TR to TR. This strategy introduces a pathway-dependent phase for different transverse partitions and thereby enables destructive interference in a steady-state gradient-echo sequence. So far, however, conventional RF spoiling schemes implicitly require that all magnetization components at a particular position acquire a constant phase in each TR. This can only be achieved by adding rewinding gradients, i.e., similar to SSFP-like sequences, either for the phase-encoding gradient in a Cartesian encoding scheme or for the variable frequency-encoding gradients in case of radial encoding. Furthermore, even if these criteria are met, the obtained signal intensities can vary from the ideally spoiled signal intensity. Lin and Song [42] therefore proposed the use of randomized RF phases in combination with strong randomized spoiler gradients to generate a steady state signal which slightly oscillates around the ideally spoiled signal. They exploited the fact that in contrast to Cartesian sampling - radial sampling schemes effectively attenuate artifacts by spreading them over the entire field of view (FoV). Especially for strong $T_{1}$-weighting, i.e., high flip angles and short TR values, the resulting signal is closer to the ideal case than achievable by conventional RF spoiling.

The purpose of this work was to investigate to which extent random RF phases may be exploited for radial MRI to obtain a pseudo steady state, i.e., a signal that fluctuates around the theoretical steady state, without the need for any timeconsuming refocusing and spoiler gradients. It is further demonstrated that the choice of a particular radial trajectory can have a major influence on the spoiling efficiency. 


\subsubsection{Spoiled FLASH Sequences}

The investigations of transverse coherences focused on three different implementations of a radial FLASH sequence. Sequence A, which serves as gold standard, used constant spoiler gradients of strength $G$ and duration $t_{\text {spoil }}$ in all spatial directions, while fully rewinding both radial frequency-encoding gradients. The sequence resulted in in-plane spoiling moments of $m=2 \pi \gamma \Delta x G t_{\text {spoil }}=\pi$ and spoiling moments in slice direction of $m=\pi \Delta z / \Delta x$, where $\Delta x$ is the pixel size, $\Delta z$ is the slice thickness and $\gamma$ is the gyromagnetic ratio. The resulting spoiling moment in slice direction RF spoiling $[37,41]$ was implemented by using a quadratic variation of $\mathrm{RF}$ and detector phase corresponding to a linear inter-pulse precession angle increment of $117^{\circ}$.

Sequence B neither employed rewinding nor additional spoiler gradients in conjunction with a constant RF transmit and receive phase. It resulted in a varying net gradient moment from TR to TR according to the gradient waveforms determined by the radial k-space trajectory. The newly proposed sequence $C$ is identical to $B$ except for a randomized RF and detector phase which was set corresponding to a random precession angle increment drawn from a uniform distribution between $-180^{\circ}$ and $+180^{\circ}$.

\subsubsection{Radial Trajectories}

All sequences employed radial k-space encoding [29, 43-45] with either a constant azimuthal displacement from one projection to the next or a periodic set of interleaved turns as described in [7]. The incremental azimuthal displacement $\phi$ could be freely chosen by the user, so that, for example, a value of $\phi=111.25^{\circ}$ refers to radial sampling according to the Golden Angle trajectory [26]. For demonstration purposes the image reconstruction was based on 233 projections to guarantee a fully sampled k-space and - being the 13th number in the famous Fibonacci sequence - to ensure a close-to-uniform distribution of the full set of projection angles [26]. Special care must be taken when constructing a radial trajectory from a constant angular displacement, since only values that lead to a full coverage of $\mathrm{k}$-space are suitable for image formation. Adding an offset of $180^{\circ}$ to $\phi$ results in identical sampling positions due to the $180^{\circ}$ symmetry (assuming symmetric echo acquisitions), while the sampling direction of every second projection is inverted. Therefore, the present investigations included both Golden Angles, the most often used value of $111.25^{\circ}$ as well as its smaller opposite angle $180^{\circ}-111.25^{\circ}=68.75^{\circ}$.

An azimuthal increment of $1 / 233 \times 360^{\circ} \approx 1.55^{\circ}$ leads to an equally spaced radial trajectory with period 233 , which renders it the smallest possible angle for 
the same set of projections. Beside its opposite angle of $181.55^{\circ}$ also a value of $7 / 233 \times 360^{\circ} \approx 10.82^{\circ}$ was considered. Because 233 is the 51 ist prime number, this choice again yields a trajectory with identical projections but in a different order. One can easily show that all angular increments of the form $n / 233 \times 360^{\circ}$ lead to the same sampling positions for all integer $n$ smaller than 233 with projections in pairwise different order.

Additional studies involved a trajectory consisting of 5 interleaved turns with 25 equally spaced projections each. The individual turns are constructed in such a way that projections from a full set of 5 turns also cover k-space uniformly. In all cases, FFT-based image reconstruction involved a gradient delay correction, compression of the multi-channel data to 10 principle components, channel-wise gridding of the raw data, density compensation, and root-sum-square combination of images.

\subsubsection{Numerical Simulations}

A framework to simulate magnetization dynamics in the presence of different radial encoding schemes was written in MATLAB (R2013a, The MathWorks, Inc., Natick, Massachusetts) using a MEX interface to a C-code implementation of a Bloch equations simulator [46]. Different gradient switching schemes were simulated by simplifying the actual gradient waveforms - as performed on the scanner - to equivalent waveforms that result in the same net gradient moment per TR. This simplification is justified by the fact that only the state of the magnetization immediately before and after an RF pulse is of any relevance, because k-space sampling and image formation was not part of the simulation. The remaining effect is the accumulation of different phase values when gradient amplitudes are changed from TR to TR which allows residual transverse magnetization components to interfere constructively or destructively depending on gradient waveforms and pixel position relative to the magnet iso-center, i.e., a position with zero gradient strength.

Simulations were performed in a pixel-by-pixel fashion assuming hard pulses and neglecting any slice profile effects. A constant proton density was assumed throughout the entire FoV. A pseudo steady state was established by assuring an RF pulse train length that exceeds five times the effective longitudinal relaxation time [24], before averaging the magnitude of transverse magnetizations immediately after the RF pulse from several TR intervals to mimic image formation. Simulations according to the Bloch equations were performed using the following parameters: $\mathrm{TR}=4 \mathrm{~ms}$, RF pulse length $10 \mu \mathrm{s}$, flip angle $30^{\circ}, \mathrm{FoV}=256 \times 256 \mathrm{~mm}^{2}$, base resolution $128 \times 128, T_{1}=283 \mathrm{~ms}$ and $T_{2}=219 \mathrm{~ms}$. Each pixel was divided into $5 \times 5$ subpixel positions to simulate intra-voxel dephasing due to different spatial positions, each of which was populated with 100 isochromates equally spaced between $-100 \mathrm{~Hz}$ and $+100 \mathrm{~Hz}$ off-resonance frequency. Gradient strength was calculated 
according to the intended trajectory relating the angle increment $\phi$ to the amplitude of the $\mathrm{x}$ - and $\mathrm{y}$-gradient during the $n^{\text {th }}$ TR-interval via

$$
\vec{G}_{n}=\left(\begin{array}{c}
G_{x, n} \\
G_{y, n}
\end{array}\right)=G_{\max }\left(\begin{array}{c}
\cos (n \phi) \\
\sin (n \phi)
\end{array}\right) \text {. }
$$

The phase $\theta_{n}$ at pixel position $\vec{r}$ acquired during this interval is then given by

$$
\theta_{n}=(\vec{r} \cdot \vec{G}) \gamma \mathrm{TR}=\cos (n \phi-\varphi) r G_{\max } \gamma \mathrm{TR}
$$

with $\varphi$ the angular coordinate of the pixel position with respect to the $0^{\circ}$ projection.

\subsubsection{MRI}

All measurements were performed on a human MRI system operating at $3 \mathrm{~T}$ (Magnetom Prisma, Siemens Healthcare, Erlangen, Germany) using a 64 channel head coil. Written informed consent, according to the recommendations of the local ethics committee, was obtained from all subjects prior to MRI. Phantom studies involved a plain water-filled phantom of the vendor doped with nickel(II) sulfate, where reference values of $T_{1}=(283 \pm 4) \mathrm{ms}$ and $T_{2}=(219 \pm 3) \mathrm{ms}$ were obtained by a Look-Locker-type IR-FLASH sequence and long-TR multi-echo spin-echo sequence, respectively. In vivo measurements of the human brain were carried out in transverse orientation at the level of the lateral ventricles to include contributions from cerebrospinal fluid with long $T_{1}$ and $T_{2}$ relaxation times. These studies used the same protocol and parameter settings $(\mathrm{TR}=4 \mathrm{~ms}$, TE $=1.12 \mathrm{~ms}$, bandwidth $=1995 \mathrm{~Hz} /$ pixel, $\mathrm{FoV}=256 \times 256 \mathrm{~mm}^{2}$, base resolution $128 \times 128$, slice thickness $8 \mathrm{~mm}$ ) as the phantom studies to allow for a direct comparison of residual artifacts.

\section{$3 \cdot 3$ RESULTS}

Figure 3.1 depicts simulated gradient-echo images which are representative for the pseudo steady state of an RF pulse train in the presence of radial encoding gradients. The simulated trajectories are either based on a constant azimuthal displacement of successive projections as indicated or correspond to a turn-based pattern, where each of 5 turns consists of 25 equally spaced projections. Because the ideal steady state, i.e., the perfectly spoiled solution, corresponds to a uniform gray image, any visible structures for sequences B (column 1 ) and C (columns 2 and 3) represent residual transverse coherences due to imperfect spoiling. In particular, for sequence B with constant RF phase all radial trajectories lead to concentric image artifacts around the simulated iso-center which exhibits a SSFP-like signal intensity as zero gradients cannot introduce phase variations in this particular position. In more detail, the actual pattern formed by hypo- and hyper-intense 


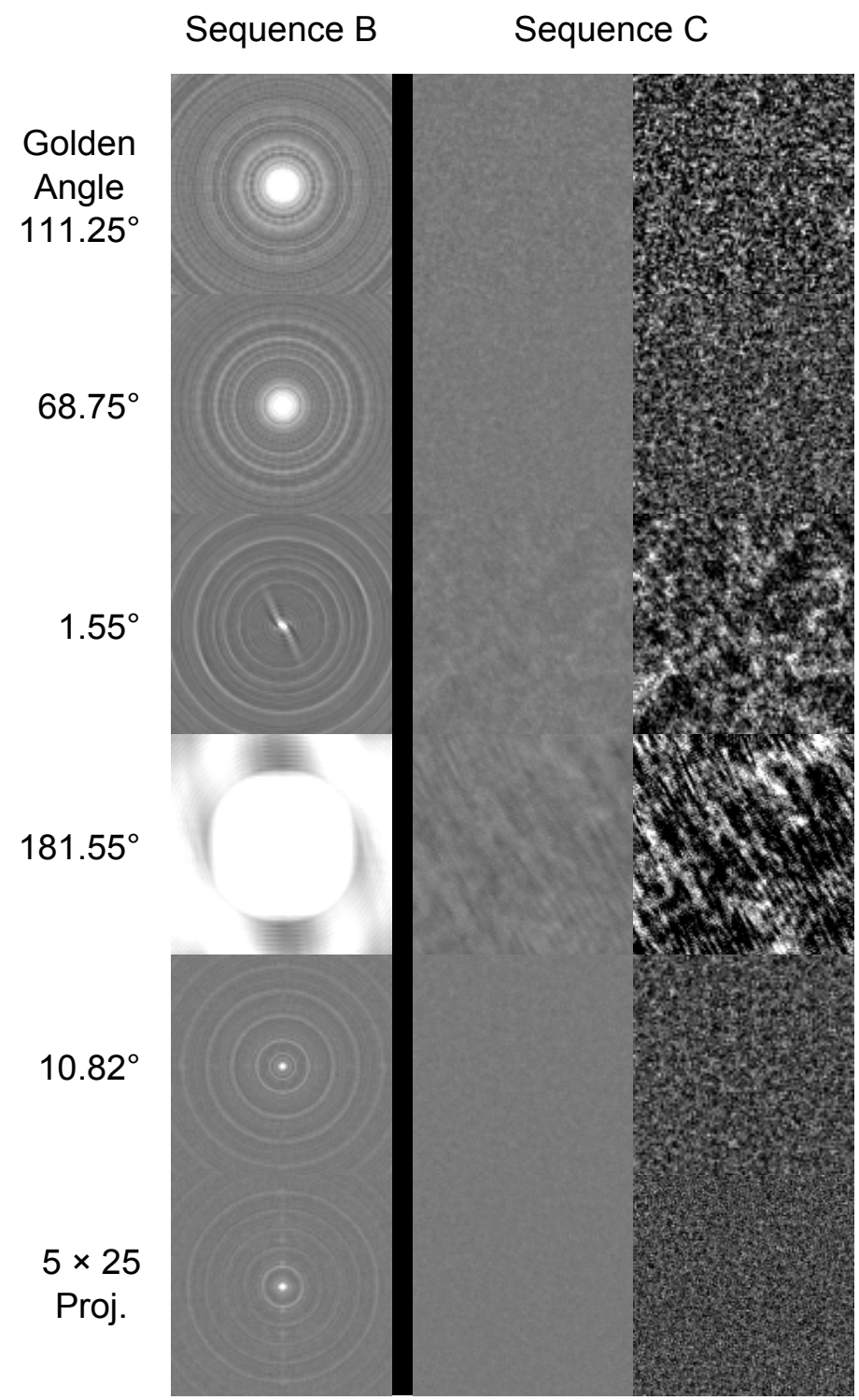

Figure 3.1: Numerical simulations of radial gradient-echo images during steady state conditions for sequence B (no radial rewinding, no spoiler gradient, constant RF phase) and sequence $C$ (as B but with random RF phases) as a function of azimuthal increment between successive projections. The bottom row corresponds to a trajectory comprising 5 interleaved turns of 25 equally spaced projections. Images in the right column are scaled by a factor of 10 . 
rings at off-center positions depends on the employed trajectory defined by the azimuthal angular increment. For example, for a value of $181.55^{\circ}$ almost the entire FoV becomes hyper-intense, while the size of the distinguished circular area around the iso-center decreases with azimuthal angular increment.

When considering the proposed sequence $C$ with randomized RF spoiling (column 2), the artifacts are very much reduced. The resulting pseudo steady state solutions are much closer to the ideal spoiling scenario with residual artifacts forming a low-intensity cloudy pattern with incoherent structure. Nevertheless, scaling of the images by a factor of 10 (column 3) still reveals slightly different spoiling performances for the trajectories studied. While the characteristic correlation length in the patterns noticeably differs, the overall (unwanted) signal intensity shows the highest artifact level for $\phi=181.55^{\circ}$ and the lowest level for the turn-based trajectory previously applied for real-time MRI $[6,8]$.

Figure 3.2 shows experimental gradient-echo images from a spherical phantom for sequences A (reference), B (no spoiling) and C (random RF). Sequence A with refocused radial gradients, constant gradient spoiler and conventional RF spoiling serves as a gold standard and clearly demonstrates the effective elimination of all artifacts independent of the chosen trajectory. In these examples no efforts have been made to compensate for coil sensitivity profiles. In close agreement with the numerical simulations shown in fig. 3.1, the results obtained for sequences $B$ and $\mathrm{C}$ identify residual artifacts from incomplete spoiling - though at a markedly different intensity. The proposed sequence $C$ exhibits the lowest artifact level for $\phi=10.82^{\circ}$, while the artifact level for $\phi=181.55^{\circ}$ is also lower than predicted by simulation.

Corresponding in vivo results for the human brain are summarized in fig. 3.3. The images represent 3 -fold zoomed versions of a transverse section covering the lateral ventricles containing cerebrospinal fluid. The subject was aligned in such a way that the magnet iso-center was close to the ventricles to allow for an observation of the spoiling efficiency in the weakest spot. In comparison to the phantom study, the artifacts are less pronounced for sequence B due to lower SNR. Nevertheless, the observation of hyper-intense ventricles for $\phi=181.55^{\circ}$ again indicates a SSFP-like refocusing of unspoiled transverse coherences which most likely reflects contributions from cerebrospinal fluid. In general, the difference images B-A and CA clearly demonstrate that the proposed sequence $C$ with randomized RF spoiling but no other precaution, i.e., without rewinders for radial gradients and without additional spoiler gradients, is superior to sequence B in its ability to suppress artifacts below noise level. This also includes removal of the signal intensity in the iso-center (arrows in fig. 3.3) which is not achieved by sequence B. Other remaining spots in the difference images C-A must be ascribed to signals from small arteries which exhibit time-dependent in-flow intensities in respective acquisitions. 


\section{$3 \cdot 4$ DISCUSSION}

Based on numerical simulations and confirmed by experimental validation this work demonstrates the superior performance of a spoiling strategy for radially encoded gradient-echo sequences which entirely relies on random RF and receiver phases. The technique is time-efficient as it eliminates the need for gradient rewinding and/or additional gradient spoilers. The analyses further show that the artifact patterns due to incomplete removal of transverse coherences in steady state gradient-echo images strongly depend on the radial trajectory - a finding which is in general agreement with earlier work [47-49]. Without rewinding gradients and for a constant RF phase, image artifacts consist of concentric rings around the iso-center characterized by zero gradient strength. This symmetry has its origin in the phase acquired per TR as given by eq. (3.2). For pixels at the same distance to the iso-center the gradient-induced phase evolution is identical up to a shift given by the angular coordinate $\varphi$. However, this latter influence is minimized by averaging data from several TR intervals. For the same distance from iso-center but two different angular coordinates $\varphi$, one can easily find a number of pulses that maps the phase evolution in a pixel very closely to the phase evolution shifted in time by these pulses: The larger the effective averaging window, i.e., the higher the number of projections per k-space, the less the influence of the angular coordinate $\varphi$. Hence, the radial symmetry of the k-space trajectory is reflected in the symmetry of artifacts in close analogy to the well-known off-center band structure for the case of Cartesian FLASH images as originally described by Crawley, Wood, and Henkelman [41]. For a constant RF phase (sequence B) the radial trajectory which emerges from an angular increment of $181.55^{\circ}$ leads to a pronounced and generalized signal increase. Because this increment is close to $180^{\circ}$, it arises as an illustrative example for a gradient echo induced by the trajectory. After two TR intervals with opposed gradient polarity the net gradient moment is close to zero at the end of the second TR interval. This refocused transverse magnetization gets mixed into the FID signal after the next RF pulse in a manner similar to a SSFP sequence where the condition of a zero net gradient moment per TR is perfectly met.

Extended Phase Graphs [49, 50] represent a different approach to describe the effect of a pulse train on a spin system. Rather than simulating single isochromates, the technique partitions the magnetization into dephased states and describes the effect of RF pulses and gradients as transitions between them. Although potentially fruitful, there are several reasons that render the approach impractical for present purposes. First, due to varying net gradient moments the dephasing state cannot be expressed by integer values and hence matrix multiplications have to be replaced by integration methods. Secondly, the number of different states grows exponentially and leads to computationally expensive calculations when simulating hundreds of RF pulses. And thirdly, difficulty arises from the fact that both spa- 


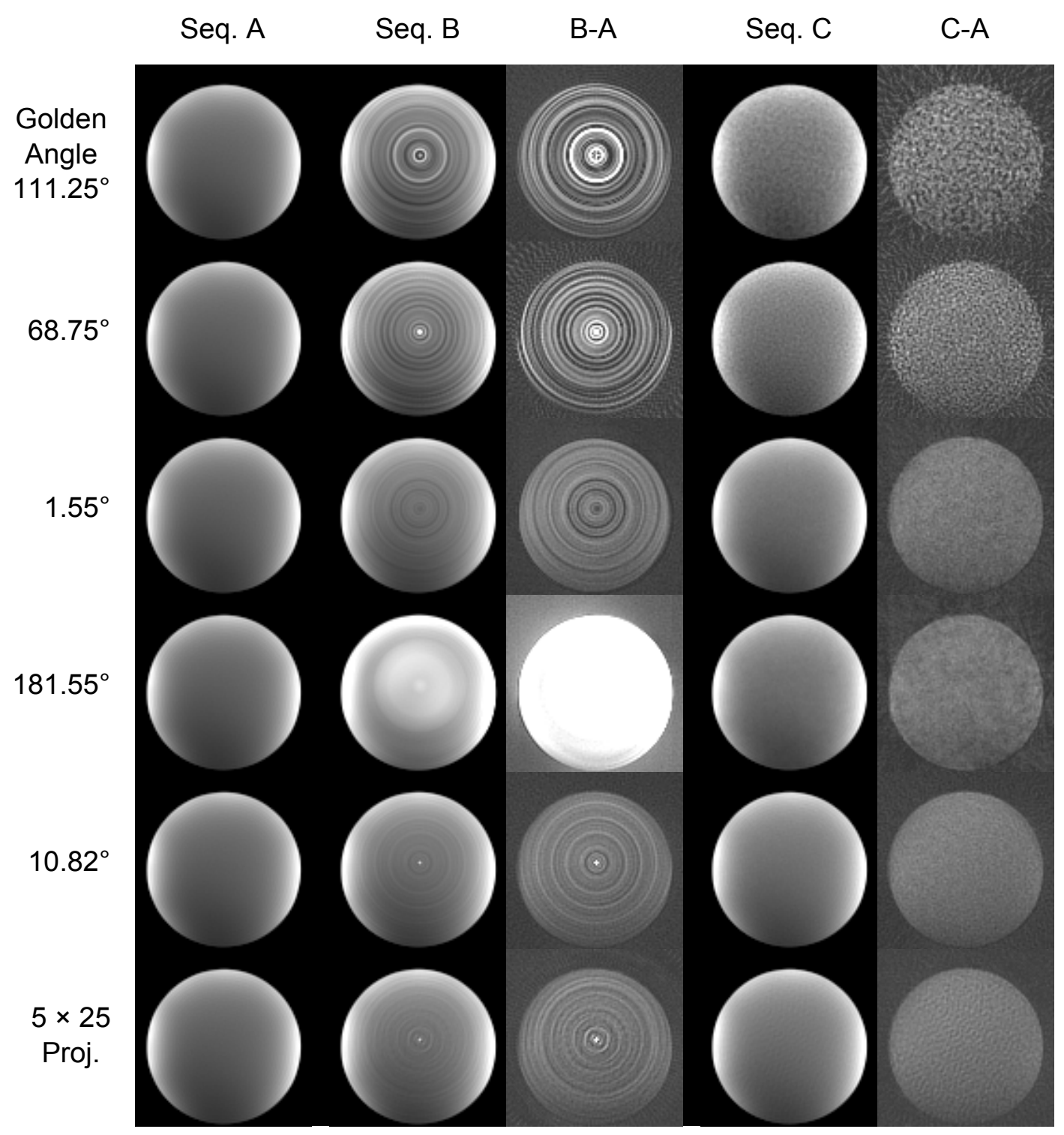

Figure 3.2: Gradient-echo images of a water-filled phantom for the reference sequence A (radial rewinding, constant spoiler gradient, conventional RF spoiling), sequence $B$ (no radial rewinding, no spoiler gradient, constant RF phase), and sequence $C$ (no radial rewinding, no spoiler gradient, random RF spoiling) as a function of azimuthal increment between successive projections as in fig. 3.1. Difference images B-A and C-A are scaled by a factor of 10 . 


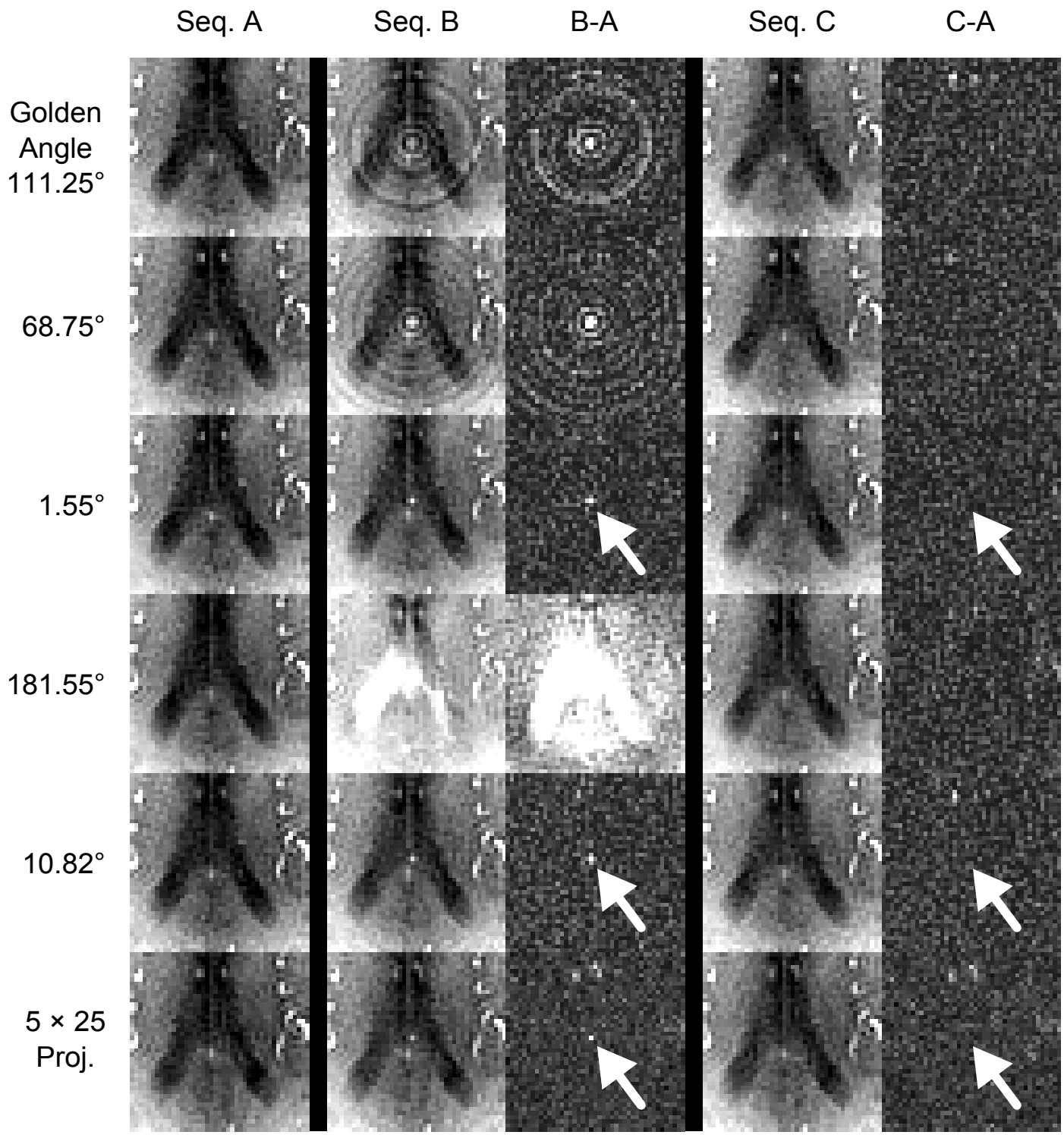

Figure 3.3: Gradient-echo images of a transverse section through the human brain at the level of the lateral ventricles for reference sequence A (radial rewinding, constant spoiler gradient, conventional RF spoiling), sequence B (no radial rewinding, no spoiler gradient, constant RF phase), and sequence $C$ (no radial rewinding, no spoiler gradient, random RF spoiling) as in fig. 3.2. Difference images B-A and C-A are scaled by a factor of 2. Arrows indicate the central artifact from unspoiled transverse coherences at the iso-center (i.e., zero gradient strength). Images in the bottom row are windowed individually. 
tial dimensions have to be incorporated when simulating a radial trajectory which extends the shift operator describing the gradient effects on different dephased states.

In conclusion, spoiling of transverse magnetizations in radial FLASH MRI may be achieved by randomized RF phases without the need for additional spoiler gradients. The approach allows for short repetition times and may be particularly attractive for high-speed real-time MRI. 
MULTI-SLICE $T_{1}$ MAPPING WITH SINGLE-SHOT

INVERSION-RECOVERY FLASH

In this chapter, an analytical expression is derived that served as a signal model in the peer-reviewed publication [25] authored by my colleague Xiaoqing Wang. We jointly developed the reconstruction methods used in the following study.

\subsection{INTRODUCTION}

Quantitative mapping of the longitudinal relaxation time $T_{1}$ is of scientific and clinical interest since it allows a comparison of MR images across subjects, MR hardware, and sequences used. $T_{1}$ maps allow tissue characterization and are of diagnostic value in a variety of pathologies including multiple sclerosis, stroke, myocardial infarction, and cancer (see [51] for a review of applications).

From the variety of $T_{1}$ mapping techniques available, Look-Locker (LL) type sequences are among the fastest since the longitudinal relaxation process is effectively interleaved with continuous image readout, e.g., by using the FLASH imaging technique. For this purpose, an initial inversion pulse is followed by a train of RF pulses with small flip angles and acquisition of the generated and spatially encoded gradient echoes. After inversion, the longitudinal magnetization changes steadily due to relaxation on the one hand, and due to the continuous application of the RF pulses on the other hand. In order to quantify the intrinsic $T_{1}$ relaxation constant one needs to find a signal model that describes how the two effects contribute to the overall signal. With this understanding, the relaxation due to the tissue-specific $T_{1}$ value can be discriminated from effects due to imaging affected by sequence parameters such as TR or flip angle.

Deichmann and Haase derived in [24] an analytical expression describing the magnetization time course in a single slice subject to imaging. A similar formula was derived by Kaptein, Dijkstra, and Tarr [52], who considered a related problem in the context of MR spectroscopy. In both cases the magnetization time course follows a mono-exponential recovery from full inversion towards the steady state given by the FLASH steady-state signal equation. This recovery is characterized by an effective relaxation time constant $T_{1}^{*}$, which is shorter than the true $T_{1}$ time. In [24] also a correction factor is given which relates the measured $T_{1}^{*}$ to the desired $T_{1}$ time. 
When considering a multi-slice measurement in which an entire stack of slices rather than a single slice is imaged, this signal model has to be extended depending on the ordering of the RF pulses. Three basic ordering scenarios are possible:

1. Sequential slices

In this scenario, all slices are measured sequentially, meaning that all frames in the first slice are acquired before switching to the second slice and so on.

2. Interleaved slices, sequential frames

Here, after the first frame in the first slice the first frame of the second slice is acquired.

\section{Interleaved slices, interleaved frames}

Here, after the first projection of the first frame in the first slice is acquired, the first projection of the first frame in the second slice is acquired.

Scenario 1 is identical to performing three single-slice LL experiments after each other where the magnetization time course in each individual experiment follows the derived model. Scenario 3 is also covered by the derived model because one particular slice still experiences a train of RF pulses with constant spacing, which is given by the product of the number of slices and the repetition time. The second scenario, however, is not compatible with the derived model, since here the train of RF pulses is interrupted whenever imaging outside the particular slice is performed. Periods of free relaxation governed by pure $T_{1}$ (when imaging is performed outside the slice) is interleaved with periods governed by $T_{1}^{*}$ (when the particular slice is imaged). But the second scenario is favored when fast imaging is required, i.e., when imaging of the entire stack has to be completed within a certain time, and when quantification of the $T_{1}$ values is based on prior reconstruction of an image series. In this case, it is advantageous to keep the temporal footprint of a single frame as small as possible to maintain temporal fidelity. By choosing a sequential rather than interleaved frame acquisition this criterion is met. However, in order to perform a proper quantification of $T_{1}$ in all slices, a proper analytical expression for multi-slice inversion-recovery is needed. This expression is derived here and its validity demonstrated in a phantom and an in vivo study.

\subsection{THEORY}

The analytical expression for single-slice inversion-recovery in [24] is based on three assumptions: Initial inversion is assumed to be perfect, $T_{2}$ relaxation is neglected since very short echo times are considered, and perfect spoiling is assumed, meaning that the magnetization vector has no transverse components immediately 
before each RF excitation. Following these assumptions, the time evolution of longitudinal magnetization $M_{z}$ can be written as a mono-exponential relaxation curve

$$
M(t)=M_{\mathrm{SS}}-\left(M_{0}+M_{\mathrm{SS}}\right) e^{-t / T_{1}^{*}}
$$

with the effective relaxation time $T_{1}^{*}=\left\{1 / T_{1}-(1 / T R) \ln \cos \alpha\right\}^{-1}$ and the steady state signal magnetization $M_{\mathrm{SS}}$, which is related to the equilibrium magnetization $M_{0}$ by

$$
M_{\mathrm{SS}}=M_{0} \frac{1-e^{-\mathrm{TR} / T_{1}}}{1-e^{-\mathrm{TR} / T_{1}^{*}}}
$$

Since $\mathrm{TR} \ll T_{1}^{*}<T_{1}$ is given in very good approximation, eq. (4.2) can be approximated to

$$
M_{\mathrm{SS}}=M_{0} \frac{T_{1}^{*}}{T_{1}}
$$

This finally allows to calculate $T_{1}$ from the measured relaxation curve directly without assumptions on the flip angle $\alpha$, which is hard to adjust correctly in vivo due to slice profile effects [53] and $B_{1}$ inhomogeneities. After fitting the three unknowns in eq. (4.1) to the measured relaxation curve, $T_{1}$ can be obtained by the Deichmann correction

$$
T_{1}=T_{1}^{*} \frac{M_{0}}{M_{\mathrm{SS}}} .
$$

This result is now used to derive a similar expression for the case of multi-slice inversion-recovery. The situation is different in the sense that the governing relaxation process depends on whether imaging is performed within the slice of interest (regime A) or outside (regime B). Figure 4.1 illustrates this situation.

In order to derive an analytical expression, we introduce two time evolution operators $U^{(A)}$ and $U^{(B)}$ which describe the evolution from time point $t_{0}$ to $t_{1}$ of the magnetization vector in these two regimes:

$$
\begin{aligned}
& M\left(t_{1}\right)=U_{t_{1}, t_{0}}^{(A)} M\left(t_{0}\right)=M_{\mathrm{SS}}+\left(M\left(t_{0}\right)-M_{\mathrm{SS}}\right) e^{-\left(t_{1}-t_{0}\right) / T_{1}^{*}} \\
& M\left(t_{1}\right)=U_{t_{1}, t_{0}}^{(B)} M\left(t_{0}\right)=M_{0}+\left(M\left(t_{0}\right)-M_{0}\right) e^{-\left(t_{1}-t_{0}\right) / T_{1}}
\end{aligned}
$$

In both regimes the initial magnetization $M\left(t_{0}\right)$ decays mono-exponentially, but relaxation time constants and steady states differ.

The magnetization at time $t_{n}$ can by described by applying the evolution operators in an interleaved manner:

$$
M_{t_{n}}=U_{t_{n}, t_{n-1}}^{(B)} U_{t_{n-1}, t_{n-2}}^{(A)} \ldots U_{t_{2}, t_{1}}^{(B)} U_{t_{1}, t_{0}}^{(A)} M\left(t_{0}\right)
$$




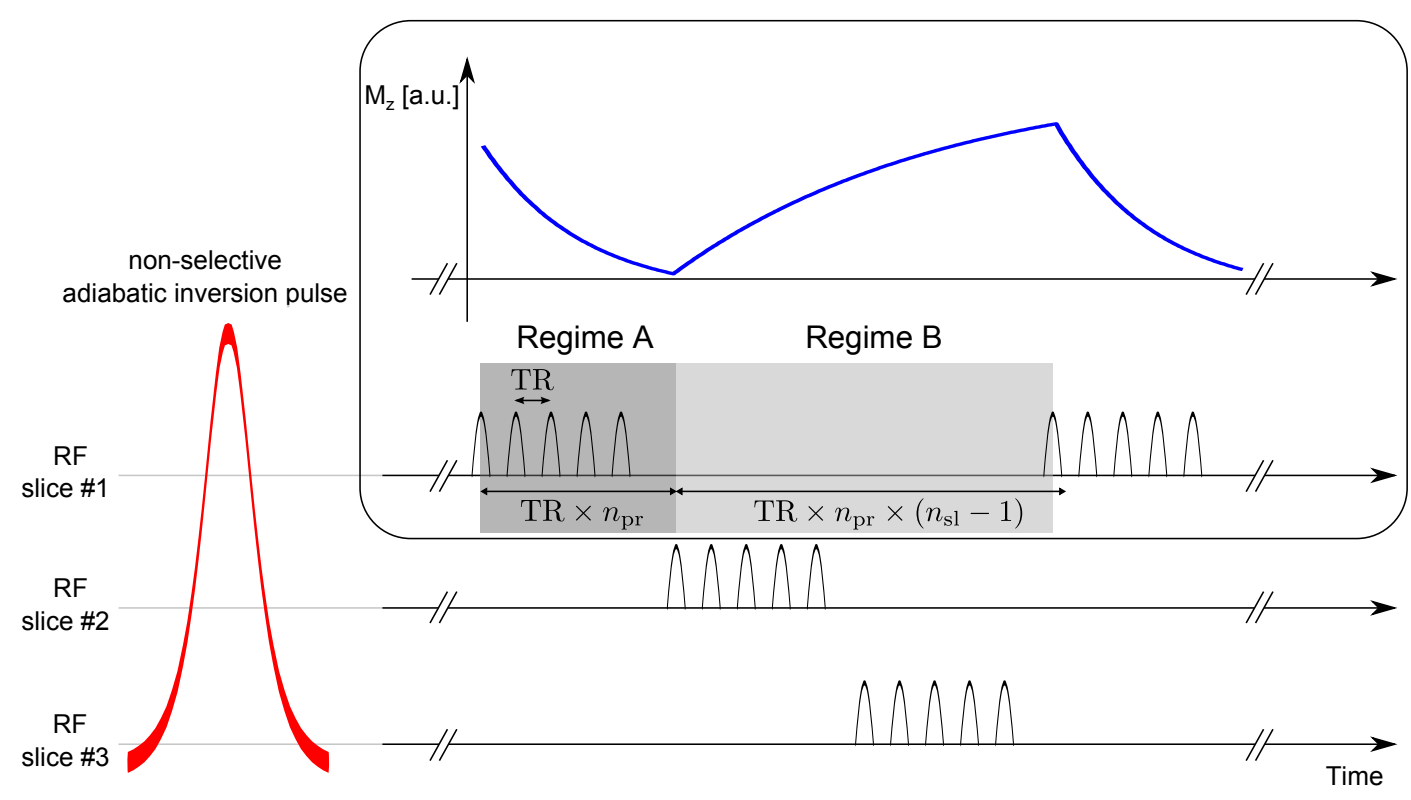

Figure 4.1: SCHEME OF A THREE-SLICE INVERSION-RECOVERY MEASUREMENT. After a nonselective adiabatic inversion pulse, imaging is performed by measuring interleaved frames in a slice-by-slice fashion. Regime A is governed by $T_{1}^{*}$ while in regime B pure $T_{1}$ relaxation takes place. Figure adapted from [25].

Let

$$
\begin{aligned}
\tau_{A} & :=n_{\mathrm{pr}} \mathrm{TR} \\
\tau_{B} & :=n_{\mathrm{pr}}\left(n_{\mathrm{sl}}-1\right) \mathrm{TR} \\
\lambda_{A} & :=e^{-\tau_{A} / T_{1}^{*}} \\
\lambda_{B} & :=e^{-\tau_{B} / T_{1}} \\
\mu_{A} & :=M_{\mathrm{SS}}\left(1-\lambda_{A}\right) \\
\mu_{B} & :=M_{0}\left(1-\lambda_{B}\right)
\end{aligned}
$$

with $n_{\mathrm{pr}}$ the number of projections per frame and $n_{\mathrm{sl}}$ the total number of slices. The constant $\tau_{A}$ is then the amount of time it takes to sample one frame in the slice, and $\tau_{B}$ the time it takes to sample the frames in the remaining $\left(n_{\mathrm{sl}}-1\right)$ slices.

Using these definitions, the magnetization at the end of each regime can be related to its respective initial value by

$$
M\left(t+\tau_{A / B}\right)=\mu_{A / B}+\lambda_{A / B} M(t)
$$


and the magnetization state after one cycle of "in-slice/off-slice" imaging can be stated as

$$
\begin{aligned}
M\left(t+\tau_{A}+\tau_{B}\right) & =\mu_{B}+\lambda_{B}\left(\mu_{A}+\lambda_{A} M(t)\right) \\
& =\mu_{B}+\lambda_{B} \mu_{A}+\lambda_{B} \lambda_{A} M(t) \\
& =\mu_{C}+\lambda_{C} M(t),
\end{aligned}
$$

where $\mu_{C}:=\mu_{B}+\lambda_{B} \mu_{A}$ and $\lambda_{C}:=\lambda_{B} \lambda_{A}<1$ were introduced. The operator expression in eq. (4.7) can be made explicit when timing according to eq. (4.8) is assumed implicitly:

$$
\begin{aligned}
M_{t_{n}} & =U^{(B)} U^{(A)} U^{(B)} U^{(A)} \ldots U^{(B)} U^{(A)} M\left(t_{0}\right) \\
& =\mu_{C}+\lambda_{C}\left(U^{(B)} U^{(A)} \ldots U^{(B)} U^{(A)} M\left(t_{0}\right)\right) \\
& =\mu_{C}+\lambda_{C}\left(\mu_{C}+\lambda_{C} M(t)\left(\ldots U^{(B)} U^{(A)} M\left(t_{0}\right)\right)\right) \\
& \vdots \\
& =\mu_{C} \sum_{i=0}^{l-1} \lambda_{C}^{i}+\lambda_{C}^{l} M\left(t_{0}\right) \\
& =\mu_{C} \frac{1-\lambda_{C}^{l}}{1-\lambda_{C}}+\lambda_{C}^{l} M\left(t_{0}\right)
\end{aligned}
$$

Here, the well-known formula for a geometric series was used from eq. (4.11d) to eq. (4.11e) and $t_{n}$ was assumed to be the time point after $l=\left\lfloor\frac{j}{n_{\mathrm{pr}}}\right\rfloor$ full "inslice/off-slice" cycles, where $j$ is the total number of $\alpha$-pulses applied within the slice of interest. To extend the expression in eq. (4.11e) to arbitrary time points, we additionally define $k:=j \bmod n_{\mathrm{pr}}$ to be the number of pulses within the slice of interest after $l$ full cycles. We can then use eq. (4.11e) to firstly determine the magnetization status at the beginning of the current imaging period and secondly calculate the final magnetization value according to eq. (4.5). This finally yields the analytical expression for the z-component of the magnetization vector at arbitrary time points in a multi-slice LL inversion-recovery experiment:

$$
M_{l, k}=M_{\mathrm{SS}}-\left[M_{\mathrm{SS}}-\left(\mu_{\mathrm{C}} \frac{1-\lambda_{\mathrm{C}}^{l}}{1-\lambda_{\mathrm{C}}}+\lambda_{\mathrm{C}}^{l} \mathrm{M}\left(t_{0}\right)\right)\right] e^{-k \mathrm{TR} / T_{1}^{*}}
$$

A structurally similar derivation can be found in [54] where an analytical expression of multi-slice spinlocking is derived, and in [Roeloffs2015a] where pulsed spinlocking for the single-slice case is considered in a similar fashion.

In an actual imaging experiment, signal intensity changes from projection to projection as given by eq. (4.12) cannot be observed as it takes multiple projections to form an image which allows spatial localization. Therefore it is appropriate to assign an effective time point to each frame that corresponds to the time point when 
the center projection was acquired. This fixes $k$ to $\hat{k}:=\left(n_{\mathrm{pr}}+1\right) / 2$ if an odd number of projections per frame is assumed. Then, a simple algebraic transformation of eq. (4.12) reveals its mono-exponential nature:

$$
\begin{aligned}
M_{l} & =M_{\mathrm{SS}}-\left[M_{\mathrm{SS}}-\left(\mu_{\mathrm{C}} \frac{1-\lambda_{C}^{l}}{1-\lambda_{C}}+\lambda_{C}^{l} M\left(t_{0}\right)\right)\right] \underbrace{e^{-\hat{k} \mathrm{TR} / T_{1}^{*}}}_{=: \xi} \\
& =M_{\mathrm{SS}}-\left[M_{\mathrm{SS} \xi}-\frac{\mu_{C} \xi}{1-\lambda_{C}}+\frac{\mu_{C} \lambda_{C}^{l} \xi}{1-\lambda_{C}}-\lambda_{C}^{l} M\left(t_{0}\right) \xi\right] \\
& =\underbrace{M_{\mathrm{SS}}(1-\xi)-\frac{\mu_{C} \xi}{1-\lambda_{C}}}_{=: \beta}+\lambda_{C}^{l} \underbrace{\left(\xi M\left(t_{0}\right)-\frac{\mu_{C} \xi}{1-\lambda_{C}}\right)}_{=:-(\gamma+\beta)} \\
& =\beta-(\gamma+\beta) e^{-\ln \left(\lambda_{C}^{-1}\right) l}
\end{aligned}
$$

This transformation clearly shows that the observable signal intensity evolution in a multi-slice experiment

a) still follows a mono-exponential relation with a slice-dependent initial value of $-\gamma$,

b) decays with a relaxation constant that depends on $T_{1}$ and sequence parameters, but is independent of the specific slice of interest, and

c) reaches the same steady state $\beta$ for all slices.

Hence, the model function describing the signal evolution stays the same when going from single-slice to multi-slice $T_{1}$ quantification, only the correction function yielding the desired $T_{1}$ values has to be adapted and takes all additional parameters such as the number of projections per frame and the number of slices into account.

\subsection{METHODS}

\subsection{1 $\quad M R I$}

All experiments were performed at $3 \mathrm{~T}$ field strength using a clinical MR scanner (Magnetom Prisma, Siemens Healthcare, Erlangen, Germany). Both, phantom and brain studies employed the standard 64-channel head coil. Volunteers without known illness were recruited and written informed consent was obtained prior to MRI. 


\begin{tabular}{ll}
\hline Parameter & Value \\
\hline FoV & $192 \times 192 \mathrm{~mm}^{2}$ \\
Matrix size & $256 \times 256$ \\
In-plane resolution & $0.75 \mathrm{~mm}$ \\
Slice thickness & $4 \mathrm{~mm}$ \\
Distance between sections & $4 \mathrm{~mm}$ \\
TR & $2.99 \mathrm{~ms}$ \\
TE & $2.01 \mathrm{~ms}$ \\
Bandwidth & $1085 \mathrm{~Hz} /$ pixel \\
Flip angle & $4^{\circ}$ \\
Projections per frame & 21 \\
Frames per slice & $150,50,30$ and 21 \\
Total acquisition time & $9 \mathrm{~s}$ \\
\hline
\end{tabular}

Table 4.1: Sequence PARAMETERS USED IN THE MULTI-SLICE $T_{1}$ MAPPING EXPERIMENT. The same set of parameters was used in both, phantom and brain study. The number of frames per slice has been adapted to match with the fixed total acquisition time shared among all multi-slice experiments.

\subsubsection{Acquisition Protocol and Phantom}

Data acquisition for single-slice and multi-slice $T_{1}$ mapping employed a LookLocker type IR sequence [24,55] which consisted of an initial non-selective adiabatic inversion pulse in combination with a spoiler gradient followed by a sliceselective radial FLASH imaging sequence (single-slice or multi-slice, respectively). Spoiling was achieved by the recently proposed method of random RF spoiling for radial FLASH ([40], see chapter 3 ) that relies on randomized phases rather than linear phase increments as used in conventional RF spoiling [37, 41]. This has the advantage that no additional gradient events are necessary, in contrast to conventional RF spoiling where a constant gradient moment per TR has to be assured. This lack of additional gradient events allows minimal TR and, consequently, maximal frame rates. Timing and sequence parameters are identical in phantom and brain study and summarized in table 4.1.

Validation of the derived multi-slice model included studies with a commercial $T_{1}$ phantom (Diagnostic Sonar LTD., Scotland, UK). This phantom contains 6 compartments with distinct $T_{1}$ values in a water environment. After image reconstruction and fitting, circular region of interests (ROIs) were drawn by hand covering about $50 \%$ of each compartment's area to avoid possible biasing of the signal by partial volume effects or ringing. For quantitative comparison, mean and standard deviation in each ROI were calculated. 


\subsubsection{Reconstruction}

To reduce the demands in memory and computation time, a software channel compression [56] was performed as an initial step after data acquisition. This method compresses the raw data from the full number of channels (given by the physical coils) down to a reduced number of virtual channels based on a principle component analysis. Here, a fixed number of eight virtual channels was used. The chosen image reconstruction algorithm nonlinear inversion (NLINV) $[6,31]$ operates on a discrete Cartesian grid whereas the raw data has been acquired in the radial domain as given by equidistant samples for multiple projections. Gridding as an interpolation process has to be performed to bring the raw data samples into the Cartesian domain. This process requires knowledge about the exact sampling trajectory in k-space, which, in principle, can be obtained from the programmed gradient waveforms. However, common imperfections in the gradient system caused by eddy currents [57-59] or timing errors in the hardware lead to deviations of the realized trajectory from the intended. In parts, these deviations can be corrected retrospectively without additional measurements. The applied method of correction is an extension of the previously introduced adaptive gradient-delay compensation [6o] in which sets of close to anti-parallel projections are used to calculate temporal delay constants. These constants are then used in the aforementioned gridding process and take into account that the true sampling positions typically deviate from the intended k-space trajectory.

Image reconstruction closely follows the procedure as described in [25] and is performed in a two-step manner: First, the trailing 10 frames of the IR image sequence are reconstructed together with their corresponding sequence of coil profiles by a first run of NLINV. Second, the coil profiles of the 1oth frame are provided as an input to the second run of NLINV in which coil profiles are treated as constant. This procedure is justified by the fact that during recovery of magnetization the dielectric properties of the imaged object remain constant and therefore no change in coil profile sensitivity is expected. Removing the coil profiles from the set of unknowns in NLINV turns the initial nonlinear problem into a linear, which is solved by means of iterative regularization and the conjugate gradients (CG) algorithm. This 2-step method ensures that signal intensity changes are exclusively reflected in the reconstructed image series, which is then subject to fitting of the derived signal model. For quantification, the reconstructed image series was fitted in a pixel-by-pixel fashion by mono-exponential curves. For simplicity, data fitting was performed on the magnitude of the reconstructed images. The three obtained parameters were than transformed into physically meaningful parameters $T_{1}^{*}, M_{\mathrm{SS}}$ and $M_{0}$. This transformation was implemented by solving a least-squares problem which only used the forward model as derived in eq. (4.13a) and rendered (a rather complex) inversion unnecessary. The desired $T_{1}$ relaxation time was fi- 
nally derived from the parameter maps by application of the original Deichmann correction $T_{1}=T_{1}^{*} \frac{M_{0}}{M_{\mathrm{SS}}}$.

\subsection{RESULTS}

Figure 4.2 shows a plot of the derived multi-slice model (eq. (4.12)) for the case of a 3 -slice experiment. $T_{1}$ and system parameter have been chosen arbitrarily to properly illustrate the observable effects in the multi-slice situation. Clearly, the temporal order in which the slices are acquired has a major effect on the dynamics, since magnetization is subject to $T_{1}$ relaxation between inversion and acquisition of the first frame. During imaging within the slice of interest both increasing and decreasing signal intensity can be found depending on whether the signal intensity has exceeded a threshold given by the single-slice steady state. A multi-slice steady state can be introduced when considering an arbitrary but fixed projection within each frame. This situation corresponds to the true imaging condition when multiple projections form a frame which is assigned to the center time point of its acquisition window. In this case, the multi-slice model can still be modeled by a mono-exponential relaxation curve (fig. 4.2 , black solid line) as predicted by eq. (4.13a).

To validate the derived model and to assess its limitations, an in vitro study was performed employing the commercial $T_{1}$ phantom described in section 4.3 . Besides single-slice imaging, a 3 -slice and a 5 -slice measurement were performed where slice positions have been chosen with respect to the 5 -slice measurement. The obtained ROI-averaged values for one slice are shown in fig. 4.3 as a function of time after inversion for both single-slice and 3-slice imaging. In both cases the zero crossings are ordered according to the corresponding $T_{1}$ values of the respective compartments. However, in the 3 -slice experiment (fig. $4.3 \mathrm{~b}$ ), apart from the obvious threefold decreased sampling rate, smaller absolutes of the initial values can be observed as well as shorter time intervals from inversion to zero-crossing. The latter effect is most prominently noticeable for the compartment with the longest $T_{1}$ value. Residual coil profile effects prevent the individual curves in the 3 -slice experiment from converging towards a common steady state. Mean and standard deviation per ROI for all obtained $T_{1}$ maps (one single-slice map and $3+5$ multi-slice maps) are found in fig. 4.4. Quantitative values for the 3-slice measurement match well with the single-slice case, but exhibit an increased standard deviation. The 5slice measurement shows on average an even further increased standard deviation and the single-slice reference values can only be reproduced for $T_{1}$ values above $500 \mathrm{~ms}$. The last two slices of this measurement fail to reproduce the smallest $T_{1}$ value of about $330 \mathrm{~ms}$ and show a trend for the next higher $T_{1}$ value.

Figure 4.5 elucidates the effect of temporal order on precision of quantification. Magnitude images of the 5-slice experiment are shown according to their overall 


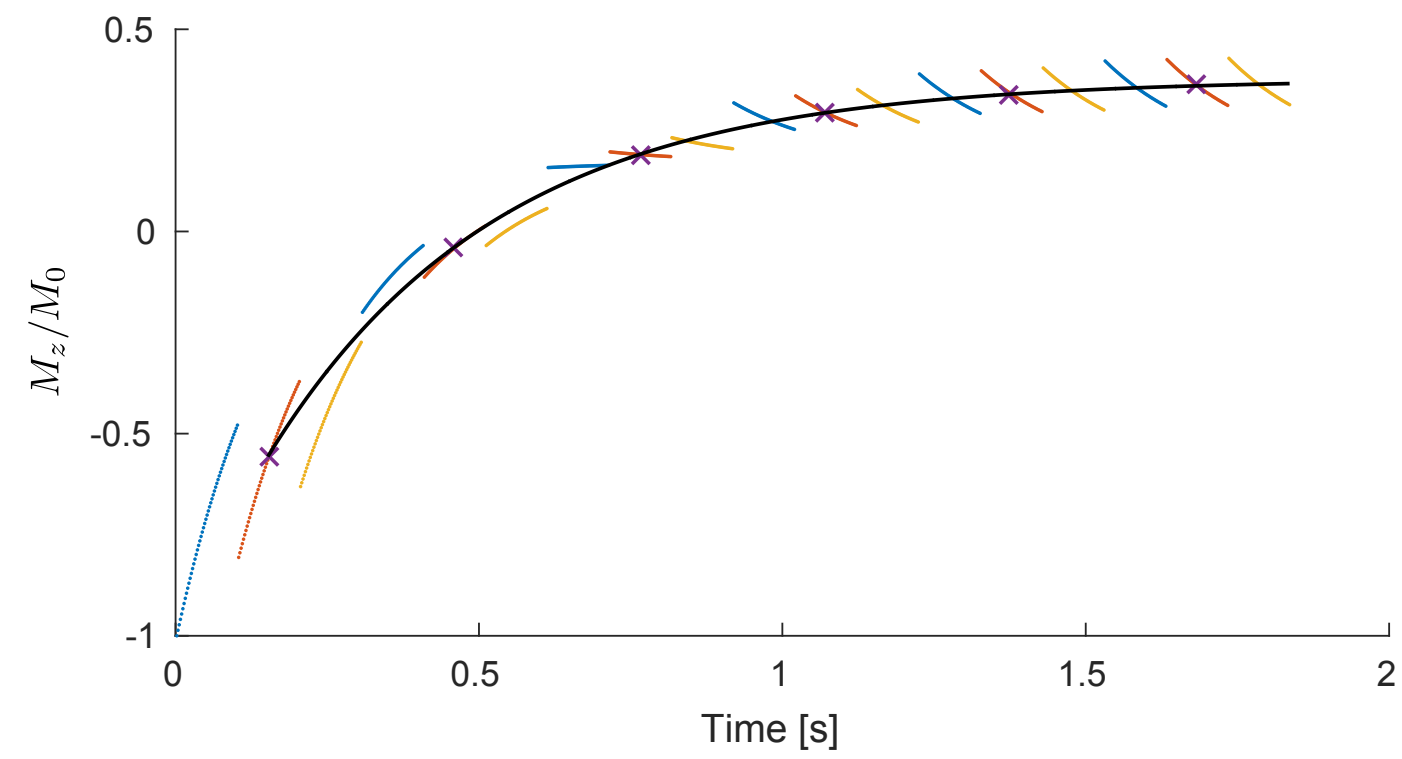

Figure 4.2: Plot of THE DERIVEd ANAlyticAl EXPRESSION FOR A 3-SLICE INVERSIONRECOVERY EXPERIMENT. The magnetization time evolution of all 3 slices (blue \#1, red \#2, yellow \#3) as given by eq. (4.12) is plotted as function of time after inversion. A mono-exponential recovery curve (black line, here only shown for slice \#2) can be found for each slice when considering a fixed projection within each frame (here: center projection, representing effective time point of each frame).

Simulation parameters: $T_{1}=1 \mathrm{~s}, \mathrm{TR}=2 \mathrm{~ms}, \alpha=8^{\circ}, n_{\mathrm{pr}}=51$

temporal order. While all slices show a similar image contrast change from inversion (bright) over zero-crossing (dark) to steady state (bright), zero-crossing in compartment 1 (left arrow) happens before acquisition of the first frame in slice 4 and slice 5. This effect does not appear for longer $T_{1}$ values, e.g. the long $T_{1}$ of about $3 \mathrm{~s}$ in the surrounding water leaves enough time to capture its dynamics in 5 to 6 frames in all slices before zero-crossing (right arrow).

The same multi-slice sequence was used for an in vivo measurement of a human brain. The reconstructed $T_{1}$ maps (fig. 4.6) show good agreement in corresponding slices. However, similar to the phantom study, with increasing number of acquired slices noise increases as well as the number of pixels where fitting failed. 


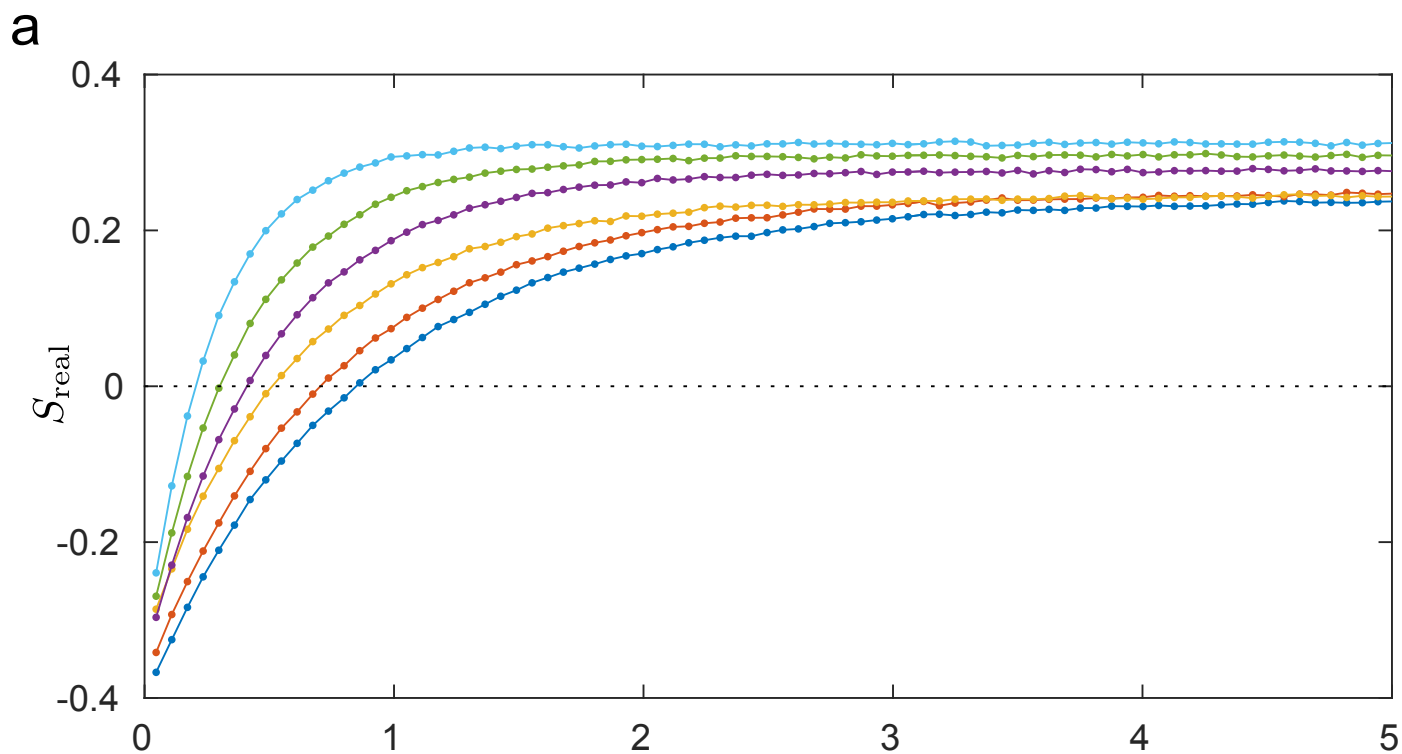

b

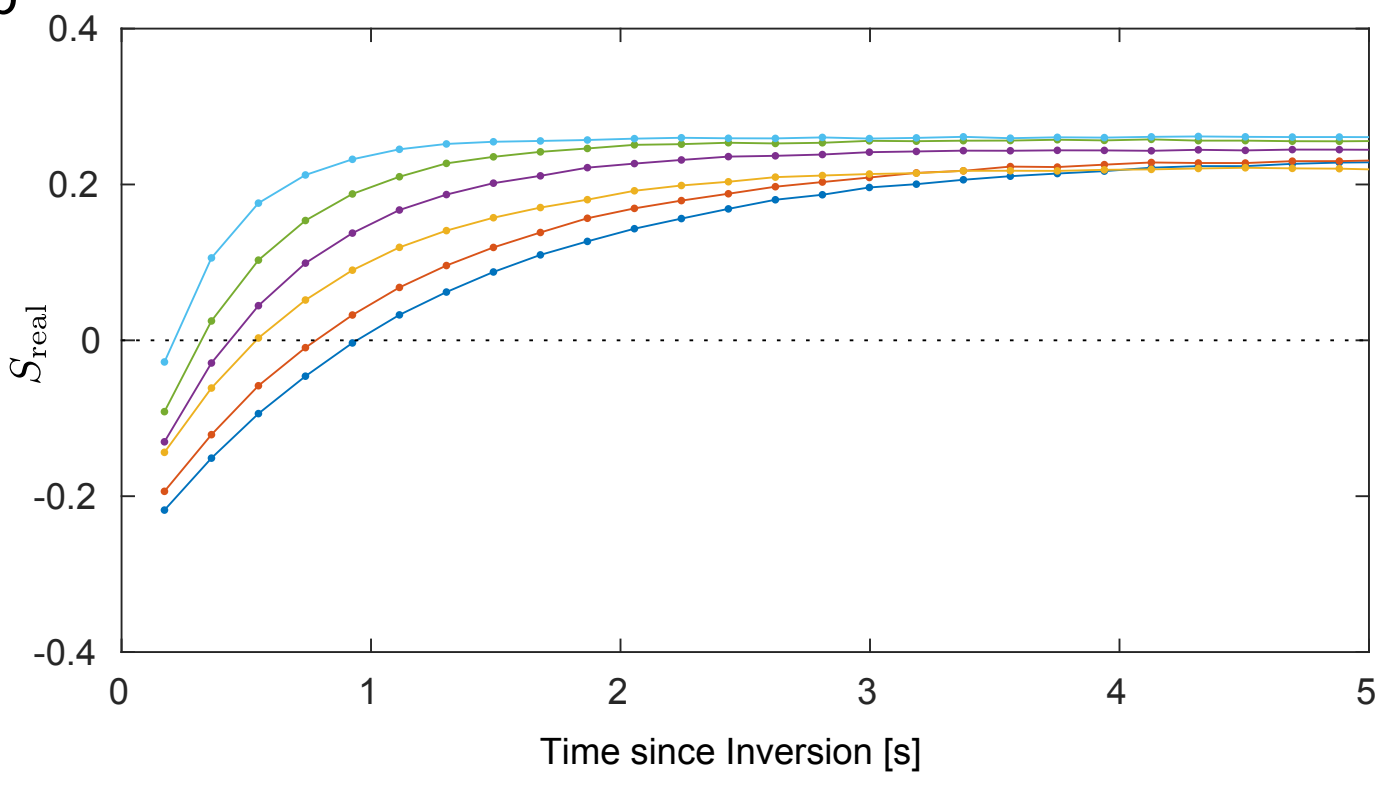

Figure 4.3: ROI-AVERAGED SIGNAL TIME COURSES FOR ALL 6 COMPARTMENTS IN THE SINGLE-SLICE (a) AND 3-SLICE MEASUREMENT (b). For better visualization only the real part of the averaged signal intensity is shown. The slice of interest was identical in both measurements. However, according to the chosen reorder scheme of the 3-slice measurement, this slice was entered last after the initial inversion. This is the source of the reduced dynamic range in (b). 


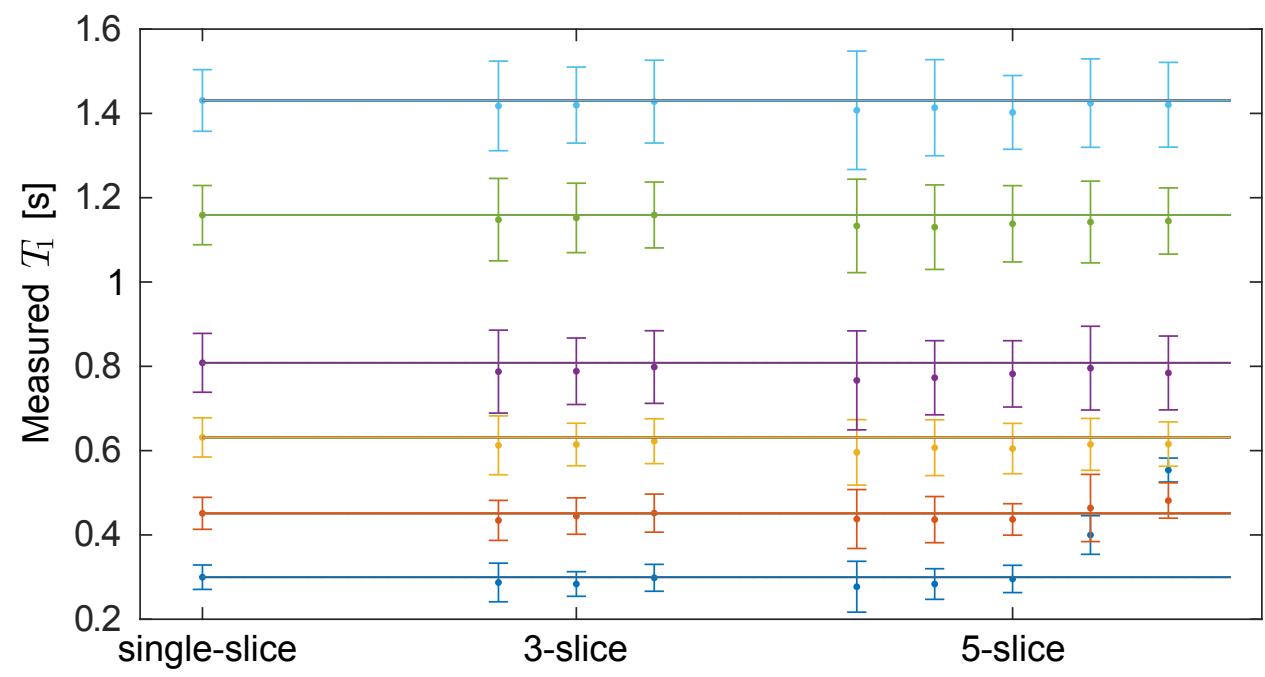

Figure 4.4: QuANTITATIVE COMPARISON BETWEEN SINGLE-SLICE AND MULTI-SLICE $T_{1}$ MAPPING. Shown are mean and standard deviation per ROI. Solid lines correspond to the single-slice case. Multi-slice data are sorted in order of acquisition. All 3-slice measurements and 5 -slice measurements for $T_{1}$ values above $0.5 \mathrm{~s}$ reproduce the singleslice values with an increased standard deviation. The last two slices of the 5-slice measurement fail to reproduce the smallest $T_{1}$ value of about $330 \mathrm{~ms}$ and show a trend for the next higher $T_{1}$ value.

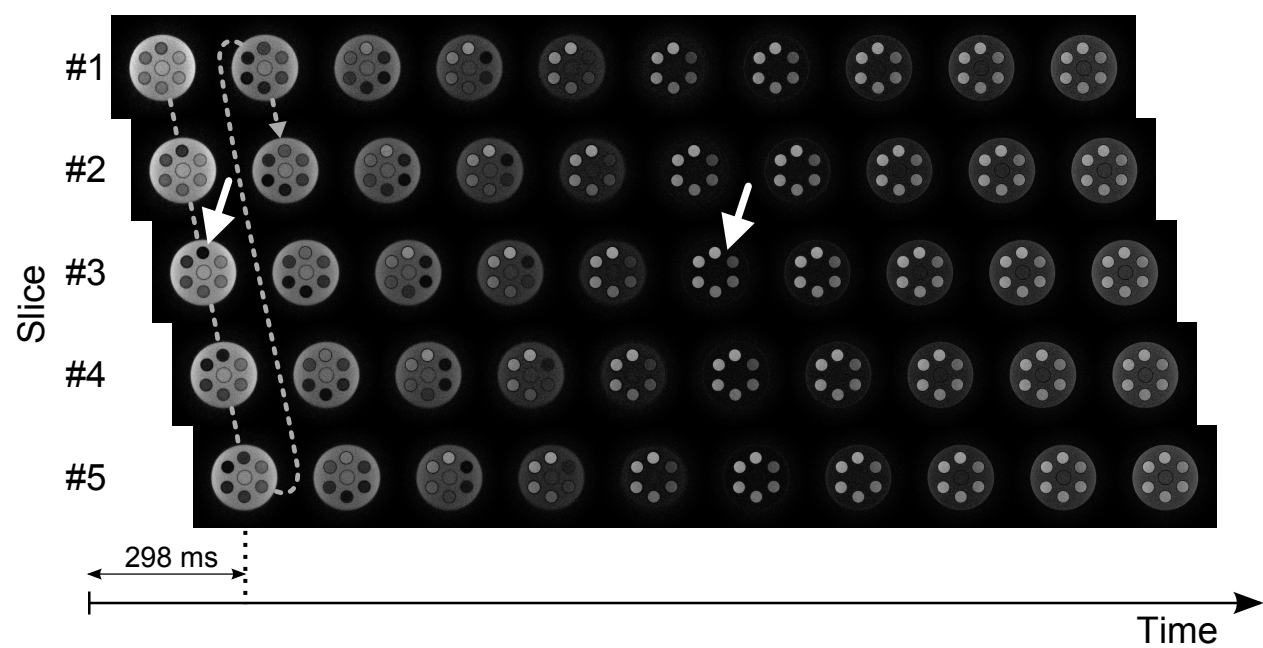

Figure 4.5: ReCONSTRUCTED IMAge SERIES OF THE 5-SLICE EXPERIMENT. While all slices show a similar image contrast change from inversion to steady state, zero-crossing in compartment 1 (left arrow) happens before acquisition of the first frames in slices \#4 and \#5 (298 ms after inversion). The long $T_{1}$ value of water leaves enough time to capture its dynamics in 5 to 6 frames in all slices before zero-crossing (right arrow). Temporal order of acquisition is indicated by the dotted gray line. 


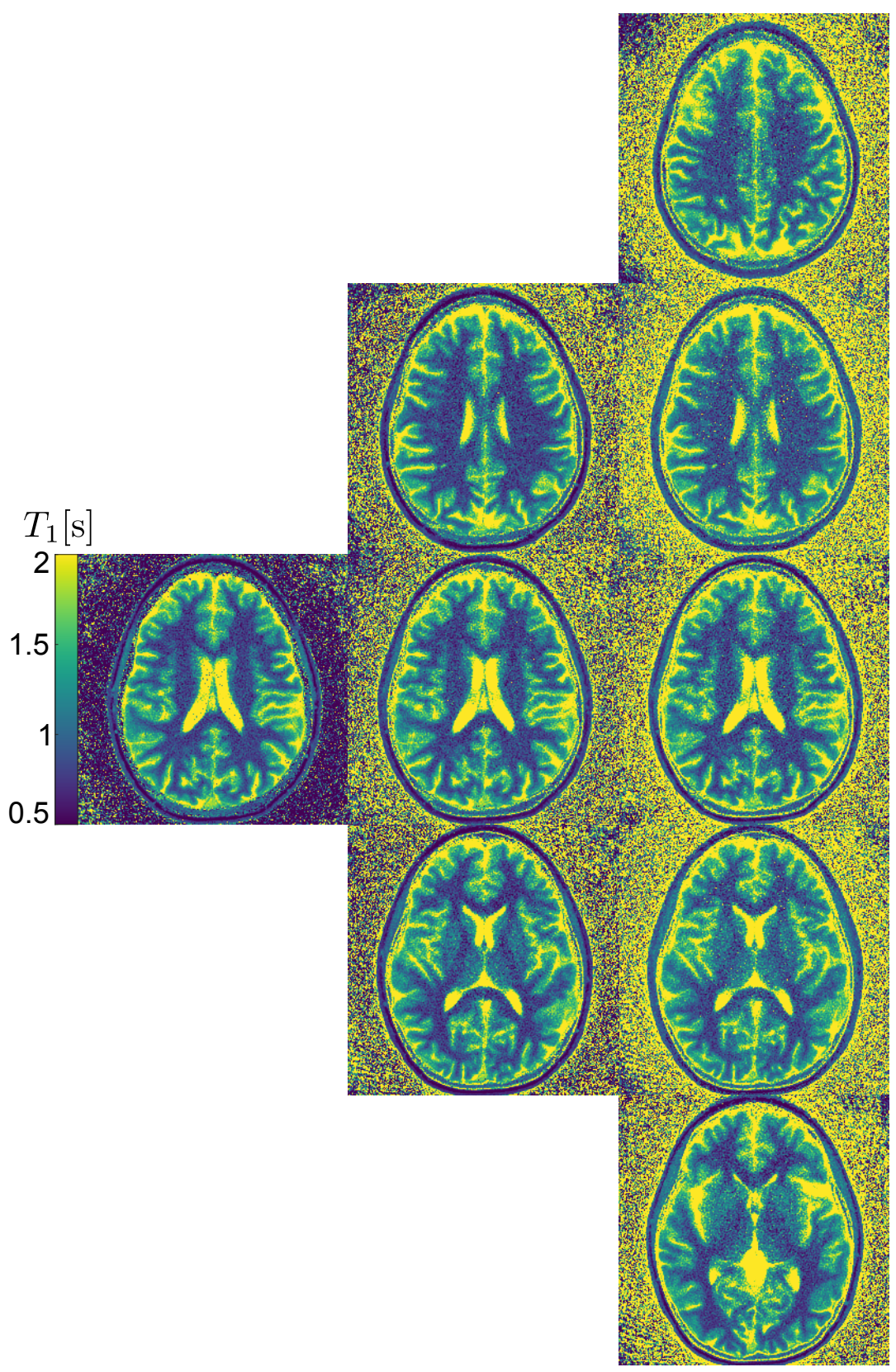

Figure 4.6: $T_{1}$ MAPS OF A HUMAN BRAIN (TRANSVERSE SECTIONS). Quantitative values obtained by pixelwise fitting of the derived signal model (eq. (4.13a)) to a single-slice (left), 3-slice (middle) and a 5-slice measurement (right). Slice positions of the singleslice and 3-slice measurement were chosen according to the 5-slice measurement. Similar to the phantom study, with increasing number of acquired slices noise increases as well as the number of pixels where fitting failed. 


\section{$4 \cdot 5$ DISCUSSION}

A novel model was developed that describes the magnetization time course in a multi-slice single-shot inversion-recovery FLASH experiment. The derived analytical expression extends the well-known Deichmann correction [24] towards a scenario in which multiple slices are acquired in an interleaved order. Under the same assumptions as the original Deichmann correction (instantaneous RF pulses, perfect spoiling, complete initial inversion, and TR $\left.\ll T_{1}^{*}\right)$ an exact solution for all slices and arbitrary starting values was presented. When considering one representative time point per frame, the observed signals can be parametrized by a 3-parameter mono-exponential recovery curve with steady state and relaxation rate independent of slice number.

This model is particularly useful for $T_{1}$ quantification based on real-time imaging, since it allows a small temporal footprint per frame while retaining the overall acquisition time of a single-slice experiment. In this study, image reconstruction was performed with NLINV, however, the model is independent of the algorithm chosen to reconstruct the image series.

In vitro studies using a commercial $T_{1}$ phantom proved validity of the multi-slice extension and further revealed general limitations of this method. While the simultaneous acquisition of 3 slices accurately reproduced the quantitative single-slice results, a severe overestimation for $T_{1}$ values shorter than $600 \mathrm{~ms}$ was found. This effect is caused by the general sequence timing and does not constitute a signal model violation. In practice, the maximal number of slices that can be simultaneously mapped is limited by several factors. Many of those are closely connected to the image reconstruction algorithm, since the latter determines the minimal number of projections per frame necessary to obtain artifact-free images at a given spatial resolution. Therefore, the maximal number of possible slices strongly depends on the selected spatial resolution, which in turn determines TR, TE, bandwidth and other parameters. However, even at minimal projections per frame, all slices have to be entered after inversion within a certain time interval, which is determined by the shortest $T_{1}$ values of interest. When this time is exceeded - as in measurements with a high number of slices - the relaxation process has already advanced to such a degree, that the dynamics in the last slices is not captured appropriately and, consequently, erroneous quantitative results are obtained. These acquisition-order-dependent effects could be mitigated by means of a slice selective rather than a non-selective inversion. However, possible inversion efficiency decrease, slice cross-talk and, most important, the necessity of a new signal model prevent an immediate transfer.

In the performed in vivo study such effects were not observed, probably because $T_{1}$ values found in the human brain at $3 \mathrm{~T}$ field strength are typically larger than $700 \mathrm{~ms}$ (see [61] for a compilation of reference values). However, the increased 
volume coverage by multi-slice imaging comes at the cost of a decreased SNR in the $T_{1}$ maps. 

MODEL-BASED RECONSTRUCTION FOR $T_{1}$ MAPPING USING SINGLE-SHOT INVERSION-RECOVERY RADIAL FLASH

The following is a reprint of the original manuscript

V. Roeloffs, X. Wang, T. J. Sumpf, M. Untenberger, D. Voit, and J. Frahm. “Modelbased reconstruction for $\mathrm{T}_{1}$ mapping using single-shot inversion-recovery radial FLASH"

which was under revision at NMR in Biomedicine at time of thesis submission. A peer-reviewed version of this article can be found at [62].

$V R$ and TS developed the reconstruction algorithm. $V R$ implemented the reconstruction algorithm and analyzed the results. $V R$ and $X W$ performed the experiments. $V R$ and $M U$ adapted the gradient delay correction method to Golden Angle sampling patterns. $D V$ implemented the sequence. $V R$ and $J F$ wrote the manuscript. 


\section{ABSTRACT}

Quantitative parameter mapping in MRI is typically performed as a 2-step procedure where serial imaging is followed by pixelwise model fitting. In contrast, model-based reconstructions directly reconstruct parameter maps from raw data without intermediate image reconstruction. Here, we propose a method that determines $T_{1}$ maps directly from multi-channel raw data as obtained by a single-shot inversion-recovery radial FLASH acquisition with a Golden Angle view order. Joint reconstruction of a $T_{1}$, spindensity, and flip-angle map is formulated as a nonlinear inverse problem and solved by the iteratively regularized Gauss-Newton method. Coil sensitivity profiles are determined from the same data in a preparatory step of the reconstruction. Validations included numerical simulations, in vitro MRI studies of an experimental $T_{1}$ phantom, and in vivo studies of brain and abdomen of healthy subjects at a field strength of $3 \mathrm{~T}$. The results obtained for a numerical and experimental phantom demonstrated excellent accuracy and precision of model-based $T_{1}$ mapping. In vivo studies allowed for highresolution $T_{1}$ mapping of human brain $(0.5 \mathrm{~mm}$ to $0.75 \mathrm{~mm}$ in-plane, $4 \mathrm{~mm}$ section thickness) and liver $(1.0 \mathrm{~mm}, 5 \mathrm{~mm}$ section) within $3.6 \mathrm{~s}$ to 5 seconds. In conclusion, the proposed method for model-based $T_{1}$ mapping may become an alternative to 2-step techniques which rely on model fitting after serial image reconstruction. More extensive clinical trials now require accelerated computation and online implementation of the algorithm. 


\subsection{INTRODUCTION}

Quantitative mapping of longitudinal relaxation is of scientific and clinical interest as $T_{1}$ relaxation times are important biophysical and physiologic indicators for tissue characterization and the assessment of perfusion, blood volume, and contrast agent uptake. The challenges for $T_{1}$ mapping are quantitative accuracy and precision as well as high spatial resolution and speed, i.e., short measurement times for routine clinical use. So far, one of the most commonly applied methods is based on a Look-Locker type acquisition sequence $[52,55]$, in which an initial inversion pulse is followed by a continuous SSFP or FLASH readout. After serial image reconstruction $T_{1}$ maps can be obtained by pixel-wise fitting.

The separation between image reconstruction and $T_{1}$ quantification in a 2-step procedure may be overcome by a so-called model-based reconstruction technique that explicitly takes into account that the measured signal follows a certain model. A potential advantage of such strategies is the better use of inherent redundancy in the data, which may be exploited by reconstructing parameter maps directly from k-space data, e.g., see [63] for a general review. This concept has previously been applied to different physical and physiological models including, for example, Cartesian $T_{1}$ and $T_{2}$ mapping [18, 19, 32], radial $T_{2}$ mapping [33], radial $T_{1}^{*}$ and $T_{1}$ mapping [20, 21], radial spin-echo DTI [22] and phase-contrast flow MRI [23].

In this work, reconstruction of $T_{1}$ maps from a single IR radial FLASH measurement is formulated as a nonlinear inverse problem and solved by the iteratively regularized Gauss-Newton method (IRGNM). Prior knowledge about the unknown parameter maps is taken into account by a parameterization of the signal model that allows for the use of variable specific regularization terms. Thus, the initially ill-posed problem can be turned into a well-posed one without inappropriately biasing the quantitative reconstruction results. The algorithm comes at moderate computational complexity due to the relatively simple Tikhonov regularization.

Validation of the proposed technique using numerical simulations, studies of an experimental phantom, and in vivo measurements of healthy subjects render this method an efficient technique for high-resolution $T_{1}$ mapping within a few seconds.

\subsection{METHODS}

\subsubsection{Signal Model and Optimization}

Following $[24,52]$ the evolution of the longitudinal magnetization $M$ over time in a Look-Locker sequence [55] is governed by

$$
M\left(M_{\mathrm{SS}}, M_{0}, R_{1}^{*} ; t\right)=M_{\mathrm{SS}}-\left(M_{\mathrm{SS}}+M_{0}\right) e^{-R_{1}^{*} t},
$$


where $M_{\mathrm{SS}}$ is the steady-state magnetization, $M_{0}$ the equilibrium magnetization and $R_{1}^{*}$ the effective relaxation rate. The latter can be written as

$$
R_{1}^{*}=R_{1}+R_{1}^{\prime}
$$

with the longitudinal relaxation rate $R_{1}=1 / T_{1}$ and a "readout" relaxation rate $R_{1}^{\prime}=-\frac{1}{\mathrm{TR}} \ln \cos \alpha$, where $\alpha$ is the effective flip angle across the slice profile and TR the repetition time. The spatial distribution of this flip angle is expected to be smooth $[64,65]$ because RF excitation was accomplished by the body coil. In order to exploit this smoothness as prior knowledge in a model-based reconstruction, a different but equivalent parameterization of eq. (5.1) is chosen which exploits the relationship $M_{0} / M_{\mathrm{SS}}=R_{1}^{*} / R_{1}$ for the case of short TR [24]:

$$
M\left(M_{\mathrm{SS}}, R_{1}, R_{1}^{\prime} ; t\right)=M_{\mathrm{SS}}\left[1-\left(\frac{R_{1}^{\prime}}{R_{1}}+2\right) e^{-\left(R_{1}^{\prime}+R_{1}\right) t}\right]
$$

Here, $M_{0}$ was eliminated and $R_{1}^{*}$ split into its components $R_{1}$ and $R_{1}^{\prime}$, which are now explicit variables. This transformation allows for an independent regularization of the two parameter maps $R_{1}$ and $R_{1}^{\prime}$ that represent two completely different sources of relaxation. For $R_{1}^{\prime}$ a similar smoothness as for $\alpha$ is expected and hence a strong smoothing regularization is appropriate for this variable.

Assuming that the magnetization time course in the experiment follows the ideal signal intensity (eq. (5.3)), the full MRI signal equation, which maps all three unknown parameters $\left(M_{\mathrm{SS}}, R_{1}, R_{1}^{\prime}\right)$ to the data acquired by the $N$ receive coils, can be stated as

$$
F: x \mapsto\left(\begin{array}{c}
P_{\vec{k}} \mathcal{F} P_{\mathrm{FoV}} C_{1} M\left(M_{\mathrm{SS}}, R_{1}, R_{1}^{\prime} ; t\right) \\
\vdots \\
P_{\vec{k}} \mathcal{F} P_{\mathrm{FoV}} C_{N} M\left(M_{\mathrm{SS}}, R_{1}, R_{1}^{\prime} ; t\right)
\end{array}\right) \text { with } x=\left(\begin{array}{c}
M_{\mathrm{SS}} \\
R_{1} \\
R_{1}^{\prime}
\end{array}\right)
$$

where $C_{j}$ are the predetermined coil sensitivity profiles, $\mathcal{F}$ is the Fourier transform, $P_{\vec{k}}$ and $P_{\mathrm{FoV}}$ are the orthogonal projections onto the measured k-space trajectory and the FoV, respectively. $T_{1}$ mapping can now be stated as a nonlinear inverse problem of the form

$$
F(x)=y,
$$

where $y$ is the raw data from all channels and for all time points along the IR process. Once the solution $x$ is found, three meaningful parameter maps can be calculated: The equilibrium magnetization (i.e., proton density), the flip angle distribution and the $T_{1}$ map.

Equation (5.5) is solved by the IRGNM (see [66] for a general reference) which considers the augmented objective function

$$
f(x)=\|F(x)-y\|_{2}^{2}+\alpha_{m}\left\|L\left(x-x^{\mathrm{ini}}\right)\right\|_{2}^{2},
$$


where $\alpha_{m}=2^{-m}$ is a regularization parameter halved in each iteration $m, x^{\text {ini }}$ a reasonable a priori estimate of $x$, and $L$ the Tikhonov regularization matrix. As $x$ comprises three different parameter maps, the general regularization matrix $L$ combines three parameter-specific regularization terms. Corresponding details are found in the Appendix (section 5.5).

\subsubsection{Constraints}

The chosen parameterization of the model (eq. (5.3)) describes the mono-exponential signal time course by two relaxation constants $R_{1}$ and $R_{1}^{\prime}$, which are physically restricted to positive real numbers. This renders the reconstruction of these parameter maps a bound-constraint problem. However, the IRGNM calculates iterates that may violate these boundary conditions. Doicu, Schreier, and Hess ([67]) presented two general modifications of the IRGNM that both integrate the bound-constraint condition into the optimization, while maintaining its peculiarities such as the descending sequence of regularization parameters. However, because these modifications require additional costly calculations, a simpler, yet effective modification was implemented: Each iterate of the IRGNM is checked for feasibility and no update in $R_{1}$ is performed in pixels where boundary constraints would be violated. These pixels are then set to the boundary value of $R_{1}$. Here, the lower bound of $R_{1}$ was set to $0.25 \mathrm{~s}^{-1}$ leading to $T_{1}$ values ranging from $0 \mathrm{~s}$ to $4 \mathrm{~s}$, hence covering common in vivo values at $3 \mathrm{~T}$ field strength.

Reconstruction techniques such as model-based relaxometry methods $[18,20]$ or MR fingerprinting [68] in general do not attempt to reconstruct images and hence no binning of k-space data to frames is necessary. Although this inherent feature allows for a perfect temporal fidelity, the computational complexity and memory demands can be significantly reduced when a certain temporal discretization is chosen. However, the largest reasonable bin size, i.e., the largest number of radial projections combined to a frame, is given by the smallest time scale on which changes are expected. For the case of $T_{1}$ mapping, this time scale is determined by the shortest effective relaxation time $T_{1}^{*}=1 / R_{1}^{*}$.

\subsubsection{Scaling}

Scaling of variables and operators has a major effect on the reconstruction results because it can change the condition number of the problem and also determines the balance between the data consistency and regularization terms. It is therefore necessary to choose a proper scaling strategy that is able to deal with different input scenarios like changes in base resolution or number of receive channels. A proper normalization and scaling should keep the same balance between data consistency and regularization terms, i.e., the chosen regularization constants should 
keep their meaning and neither depend on application nor be adapted to the actual dataset. In contrast to other model-based methods where the unknowns itself are explicitly scaled $[19,23,32]$, this work ensures a proper scaling by only normalizing the raw data, the point spread function (PSF) operator, and the coil vector. Details can be found in the Appendix (section 5.5). This normalization scheme ensures that the energy ratios of the gradients stay approximately constant, independent of the number of channels, the chosen base resolution, or the number of data frames available (for example by choosing a different bin size).

\subsubsection{MRI}

All MRI studies were performed at $3 \mathrm{~T}$ using an MRI system with $80 \mathrm{mT} / \mathrm{m}$ gradients (Magnetom Prisma, Siemens Healthcare, Erlangen, Germany). Brain studies were performed with the standard 64-channel head coil, while abdominal applications employed the 18-channel thorax coil in combination with suitable segments of the 32-channel spine coil. Volunteers without known illness were recruited from the local university. Written informed consent, according to the recommendations of the local ethics committee, was obtained from all subjects prior to MRI.

Experimental validations were performed with use of a commercial $T_{1}$ phantom (Diagnostic Sonar LTD., Scotland, UK) consisting of 6 compartments with defined $T_{1}$ values surrounded by water. An IR fast spin echo (FSE) sequence with 13 logarithmically spaced inversion times between $50 \mathrm{~ms}$ and $2300 \mathrm{~ms}$ served for $T_{1}$ determination $(\mathrm{TR}=7.2 \mathrm{~s}$, measuring time $=50 \mathrm{~min}$ ).

Data acquisition for $T_{1}$ mapping employed a Look-Locker type IR sequence [24, 55]. An initial inversion module consisting of an adiabatic, non-selective inversion pulse and a spoiler gradient is followed by a slice-selective radial FLASH readout. Spoiling was achieved by utilizing a recently proposed method [40] that relies on random phases of the RF pulses, whereas conventional RF spoiling in a radial sampling scheme requires a time-costly incorporation of gradients to obtain a constant gradient moment.

The view order of the continuously acquired radial projections is based on a constant angular increment of about $68.75^{\circ}$. This angle increment represents the supplementary angle of the widely used Golden Angle of about $111.25^{\circ}$ [26] and achieves the same k-space coverage with reversed direction of rotation due to the central symmetry of the resulting patterns. However, the smaller angle increment was shown to result in a slightly superior spoiling efficiency when combined with random RF pulse phases [40]. Experimental details for acquisitions of the human brain and liver are summarized in table 5.1.

At this stage, model-based reconstructions were implemented on a single graphics processing unit (GeForce GTX TITAN, NVIDIA, Santa Clara, CA) using the MATLAB Parallel Computing Toolbox (R2015b, The MathWorks, Inc., Natick, Mas- 


\begin{tabular}{|c|c|c|c|}
\hline & \multicolumn{2}{|c|}{ Brain } & Abdomen \\
\hline $\mathrm{FoV} / \mathrm{mm}^{2}$ & $192 \times 192$ & $192 \times 192$ & $320 \times 320$ \\
\hline Image matrix size & $256 \times 256$ & $384 \times 384$ & $320 \times 320$ \\
\hline In-plane resolution $/ \mathrm{mm}^{2}$ & $0.75 \times 0.75$ & $0.5 \times 0.5$ & $1.0 \times 1.0$ \\
\hline Section thickness / mm & 4 & 4 & 5 \\
\hline $\mathrm{TR} / \mathrm{ms}$ & 2.99 & 3.35 & 2.40 \\
\hline Bandwidth / Hz pixel ${ }^{-1}$ & 1085 & 685 & 1420 \\
\hline Nominal flip angle $/{ }^{\circ}$ & 4 & 4 & 4 \\
\hline Total number of projections & 1491 & 1491 & 1491 \\
\hline Total acquisition time / s & 4.5 & 5.0 & 3.6 \\
\hline
\end{tabular}

Table 5.1: ACQUISITION PARAMETERS FOR MODEL-BASED $T_{1}$ MAPPING.

sachusetts), and performed offline after data acquisitions. Reconstruction times ranged from minutes to hours, depending on whether data size allowed a computation entirely on the GPU device.

To allow for a critical analysis of image quality, all maps were obtained without masking/thresholding of low-intensity pixels as well as without any spatial filtering.

\subsubsection{Numerical Simulation}

A numerical phantom was designed to investigate the robustness of the reconstruction algorithm with respect to undersampling and noise. It consisted of 3 circular tubes in a circular surrounding, where all compartments shared the same proton density, but differed in $T_{1}$ relaxation time from 0.4 to $0.8,1$ and $3 \mathrm{~s}$ (see fig. 5.2). This simple geometry allowed for an analytic calculation of the Fourier transform function at arbitrary time points. Sampling in k-space was therefore mimicked by simply evaluating the Fourier transform function at points given by the radial k-space trajectory; a simulation strategy that preserves truncation effects such as ringing. The magnetization time course in each compartment was simulated to follow the ideal signal as given by the model function. The simulated flip angle was set to $3.2^{\circ}$ for the entire FoV. Image matrix size, TR and the total number of projections were chosen identical to the brain protocol (table 5.1, first column). Complex white Gaussian noise with a standard deviation corresponding to about $2 \%$ of the entire signal intensity in k-space was added to the raw data. The view-order scheme was identical to the implementation on the scanner using a constant angular displacement of $68.75^{\circ}$ from projection to projection. To determine the maximal number of projections that can be binned to one time point without mitigating quantitative accuracy, the numbers for the projection per frames were chosen to follow the Fibonacci series as these integers lead to a close-to-uniform distribution in the 
context of Golden Angle sampling [26]. Numerical simulations were performed without parallel imaging simulating only one homogeneous receive coil.

\subsubsection{Preprocessing}

To correct for deviations from the intended k-space sampling trajectory due to imperfections in the gradient system, a gradient delay correction was applied prior to gridding. This correction is an extension of the adaptive gradient-delay compensation presented in [6o]. It estimates and corrects for parallel shifts with respect to the realized radial traversal of $k$-space.

The proposed reconstruction method uses information from multiple coil elements as present in coil arrays for parallel imaging. However, due to the iterative approach in this method, a high number of coils leads to a tremendous increase in memory and computation time. Therefore, the same software channel compression technique [56] was performed as already successfully applied in our real-time applications $[6,9,12]$. It reduces the amount of raw data by introducing a smaller number of virtual channels which are obtained by a principle component analysis. In this study the number of virtual channels was fixed to 8 .

\subsubsection{Determination of Coil Sensitivity Profiles}

The proposed model-based reconstruction requires knowledge of the coil sensitivity profiles because they are an explicit part of the signal model but not treated as unknowns. Their determination is a relatively simple task, since they can be assumed to be constant during the entire relaxation process and - in principle the full data set can be used for estimation. Coil profiles are determined by the nonlinear inversion (NLINV) method [6] in the same fashion as already successfully applied in the context of fast $T_{1}$ mapping [25]. Of course, even with known coil sensitivities the reconstruction problem remains highly nonlinear due to the inherent nonlinearity of the IR signal model.

\section{$5 \cdot 3$ RESULTS}

\subsubsection{Validation}

The effect of binning was investigated to determine the largest possible number of projections per frame. The results for a numerical phantom are shown in fig. 5.1 depicting $T_{1}$ as a function of the number of radial projections per frame (i.e., bin size) together with the respective relative error for each compartment. Quantitative evaluation was performed on $T_{1}$ maps after 14 Gauss-Newton steps. Accuracy 


\section{a}

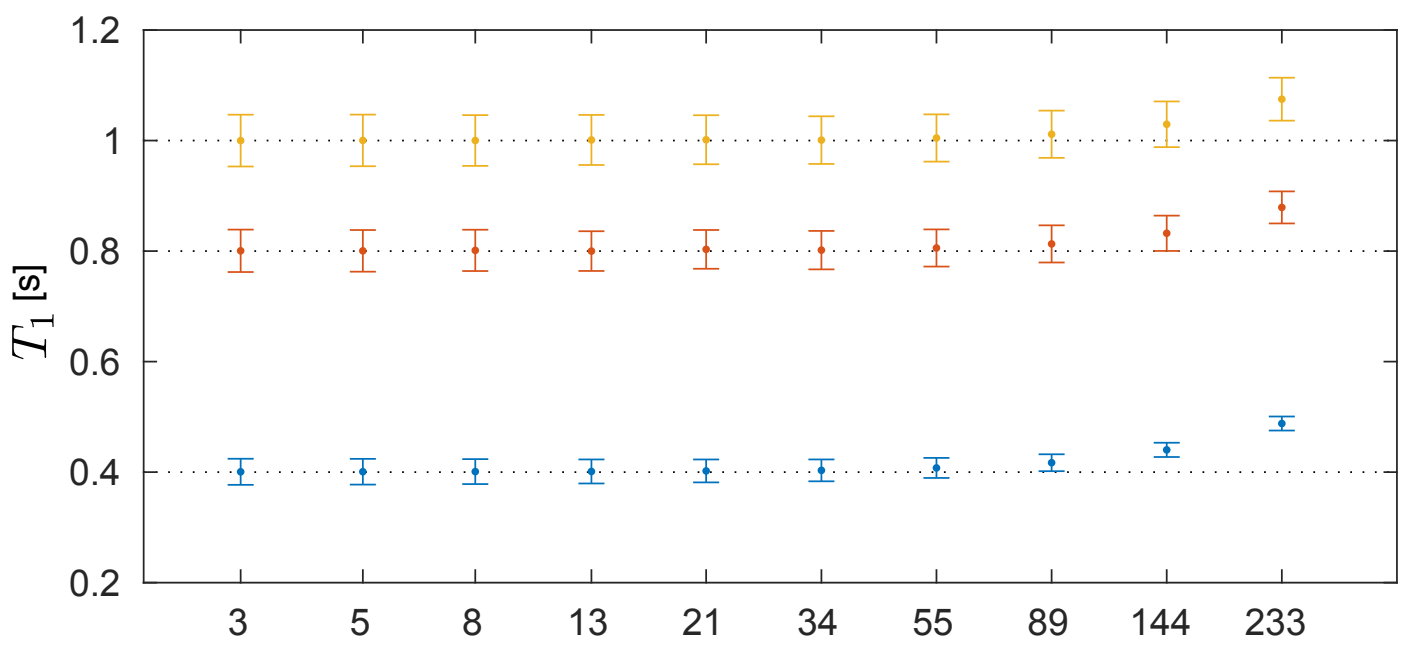

b

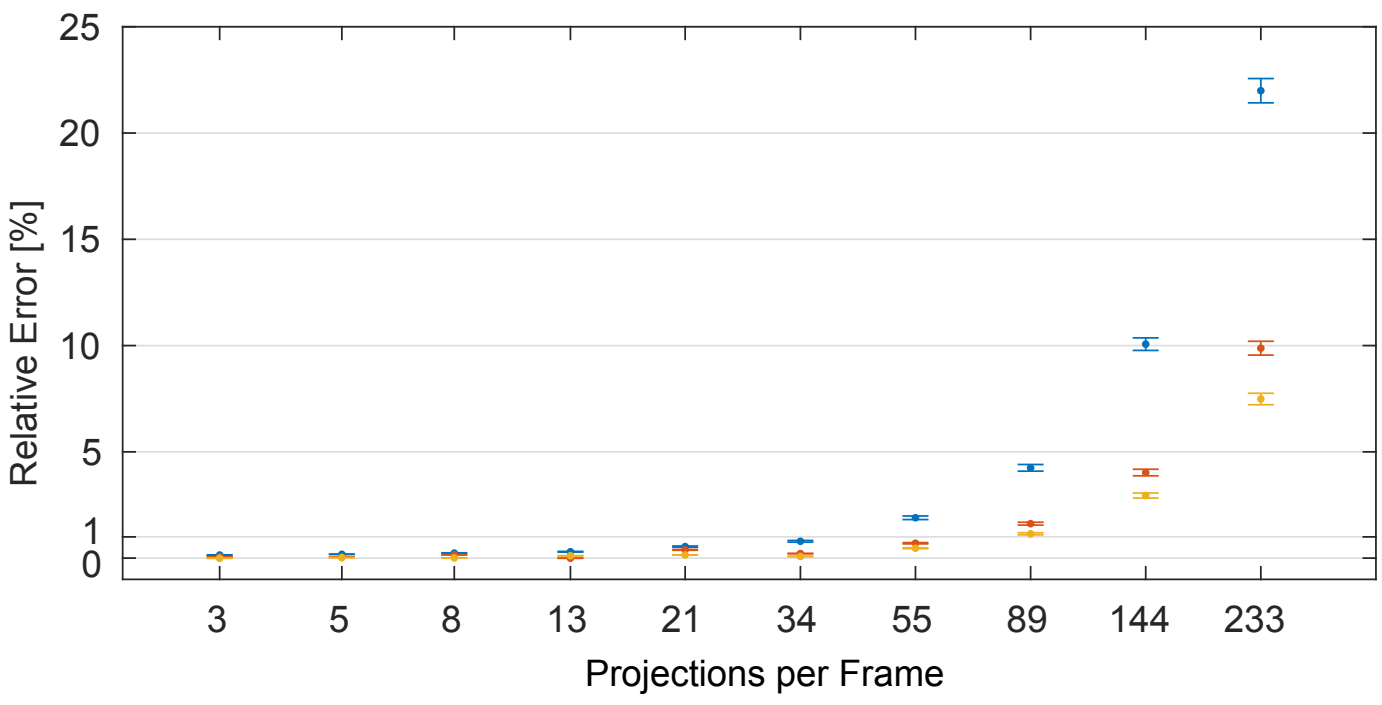

Figure 5.1: Model-based $T_{1}$ MAPping OF SIMUlated Data. (a) Mean $T_{1}$ and SD per ROI as a function of number of projections per frame and (b) corresponding relative error after 14 Gauss-Newton iterations. All reconstructions were performed using the same raw data. Accuracy increases monotonically with decreasing bin size for all three $T_{1}$ values and deviations are less than $1 \%$ for all $T_{1}$ values for bin sizes smaller or equal to 34 projections per frame. 
increases monotonically with decreasing bin size for all three $T_{1}$ values. It turned out that deviations were less than $1 \%$ for all $T_{1}$ values for bin sizes smaller or equal to 34 projections per frame. Therefore, a fixed bin size of 21 projections per frame was chosen for all subsequent reconstructions, which represent temporal footprints of $21 \times$ TR $(=50,63$, and $70 \mathrm{~ms}$, respectively) per frame.

Figure 5.2 addresses the general convergence of the model-based IRGNM. The three iteratively estimated parameter maps are shown together with the corresponding physical maps for each Gauss-Newton step. With increasing number of iterations the regularization strength is decreased, while parameter maps become sharper before finally noise starts to build up, most prominent in $R_{1}$ and $T_{1}$ maps. Like in most other regularization-based methods, no attempt has been made to determine the optimal strength of regularization automatically. Instead, the corresponding number of Gauss-Newton steps was found heuristically.

Figure 5.3a shows the reconstructed physical maps of a $T_{1}$ phantom after 11 Gauss-Newton steps. The flip angle map is a spatially smooth distribution with average values markedly below the nominal angle of $4^{\circ}$ due to the influence of the slice profile. The quantitative evaluation in fig. $5.3 \mathrm{~b}$ demonstrates that 11 iterations lead to reliable values for all $T_{1}$ values between $0.3 \mathrm{~s}$ to $1.5 \mathrm{~s}$. Figure $5.3 \mathrm{C}$ confirms excellent accuracy and precision of the proposed reconstruction algorithm by a comparison with a long-TR Cartesian IR-FSE sequence with multiple inversion times.

\subsubsection{In Vivo $T_{1}$ Mapping}

Figure 5.4a shows maps of $T_{1}$, proton density and flip angle for a transverse section of the brain of a normal subject. Corresponding maps of two abdominal sections covering liver and kidney are depicted in fig. $5.4 \mathrm{~b}$. Figure 5.5 demonstrates the ability to obtain $T_{1}$ maps of the brain at even higher spatial resolution of $0.5 \times 0.5 \mathrm{~mm}^{2}$ in plane and $4 \mathrm{~mm}$ section thickness. Table 5.2 summarizes quantitative $T_{1}$ values obtained for various tissues. The values are in general agreement with literature data obtained at $3 \mathrm{~T}$. However, for frontal gray matter the proposed method yields about $300 \mathrm{~ms}$ longer $T_{1}$ values than previous reports. 


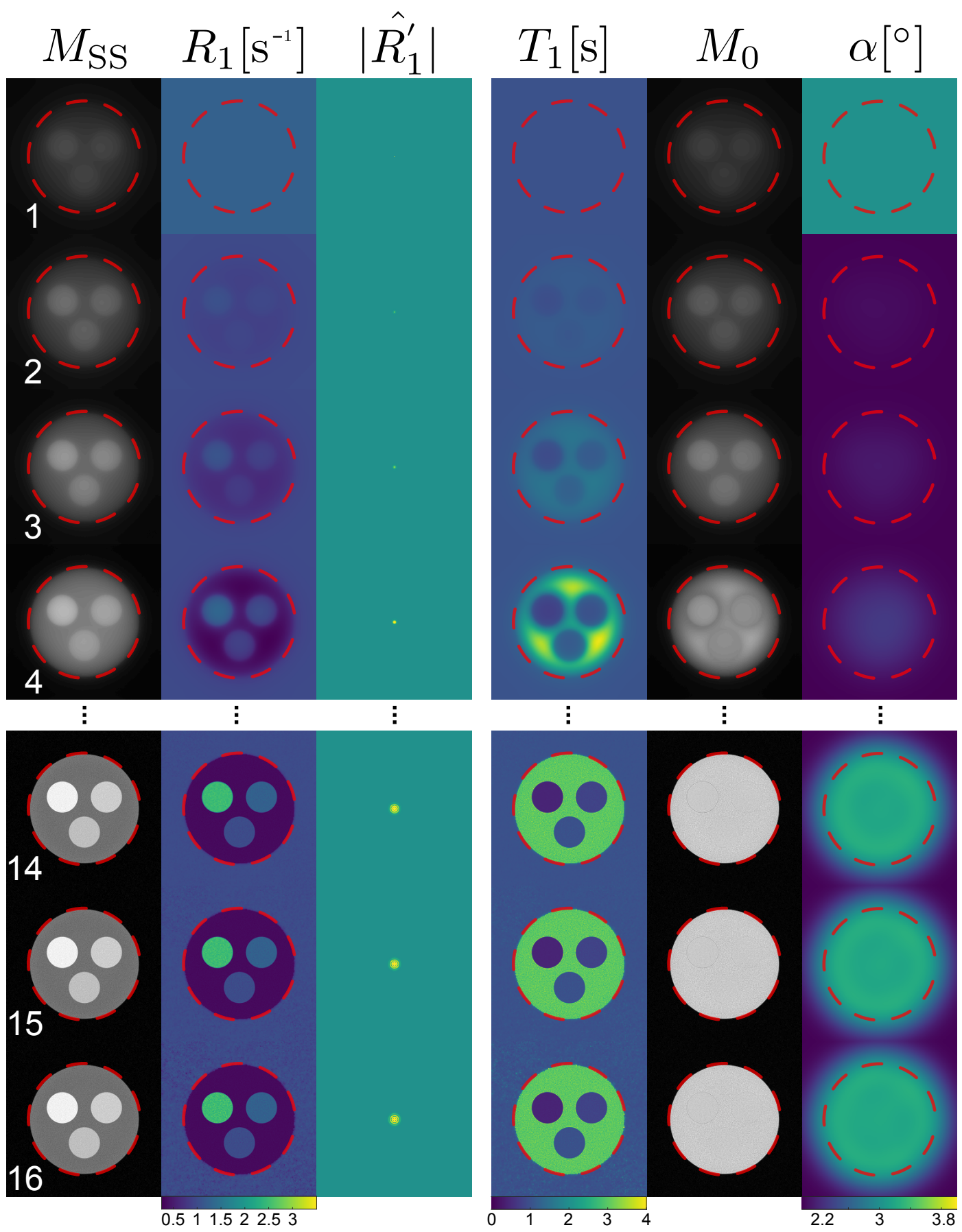

Figure 5.2: (Left) PARAMETER MAPS AND (RIgHT) DERIVEd PHYSICAL QUANTITIES $T_{1}$, SPIN DENSITY $M_{0}$, AND FLIP ANGLE $\alpha$ DURING ITERATIVE ESTIMATION (TOP TO BOTTOM). For improved visibility, the support of the circular object is marked (red circle). The regularization strength is halved in every Gauss-Newton step. With increasing number of iterations sharpness improves before noise amplifies $\left(R_{1}\right.$ and $T_{1}$ maps). 


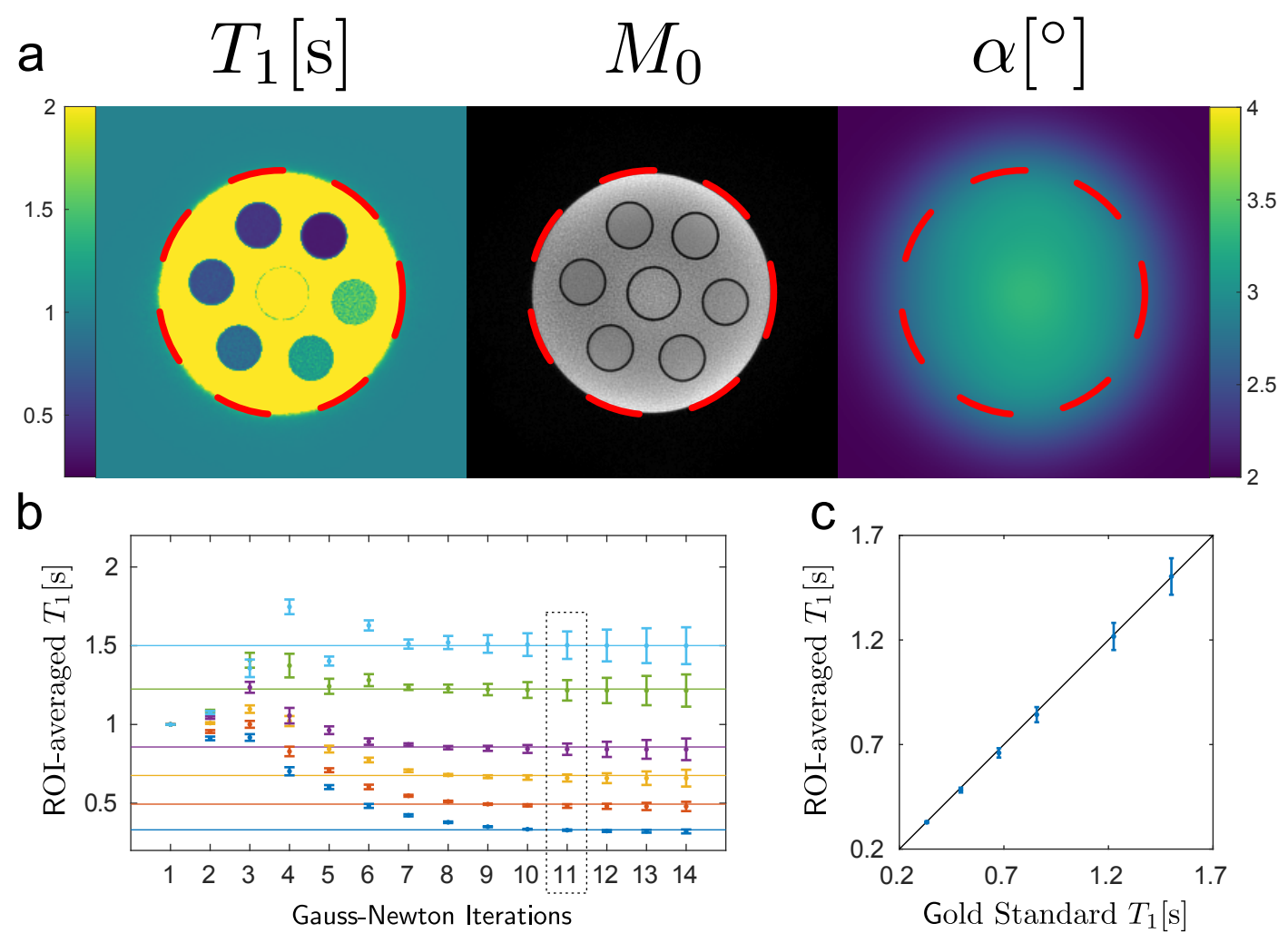

Figure 5.3: MODEL-BASEd $T_{1}$ MAPPING OF A PHANTOM WITH 6 COMPARTMENTS COVERING $T_{1}$ VALUES FROM $0.3 \mathrm{~s}$ TO $1.5 \mathrm{~s}$. (a) Maps of physical parameters after 11 Gauss-Newton steps, (b) the convergence behavior of the mean $T_{1}$ per ROI, and (c) a comparison of the $T_{1}$ value for the preferred iteration vs a multi-IR FSE measurement (gold standard). For improved visibility, the support of the circular object is marked (red circle).

\begin{tabular}{lcll}
\hline Tissue & $T_{1}{ }^{1} / \mathrm{ms}$ & Literature / ms & \\
\hline Frontal WM & $788 \pm 45$ & $699-985$ & {$[69-72]$} \\
Occipital WM & $801 \pm 44$ & $758-940$ & {$[69,72,73]$} \\
Frontal GM & $1554 \pm 119$ & $1209-1322$ & {$[69,73]$} \\
Occipital WM & $1369 \pm 112$ & $1283 \pm 37$ & {$[73]$} \\
& & & \\
Liver & $804 \pm 83$ & $767-812$ & {$[74,75]$} \\
Kidney Cortex & $1394 \pm 177$ & $1142-1375$ & {$[75,76]$} \\
\hline
\end{tabular}

Table 5.2: $T_{1}$ RELAXATION TIMES (SINGLE SUBJECT) IN BRAIN $\left(0.75 \times 0.75 \times 4 \mathrm{~mm}^{3}\right.$ RESOLUTION), LIVER AND KIDNEY $\left(1.0 \times 1.0 \times 4 \mathrm{~mm}^{3}\right)$.

${ }^{1}$ Mean and SD per ROI, ${ }^{2} \mathrm{WM}=$ white matter, ${ }^{3} \mathrm{GM}=$ gray matter. 


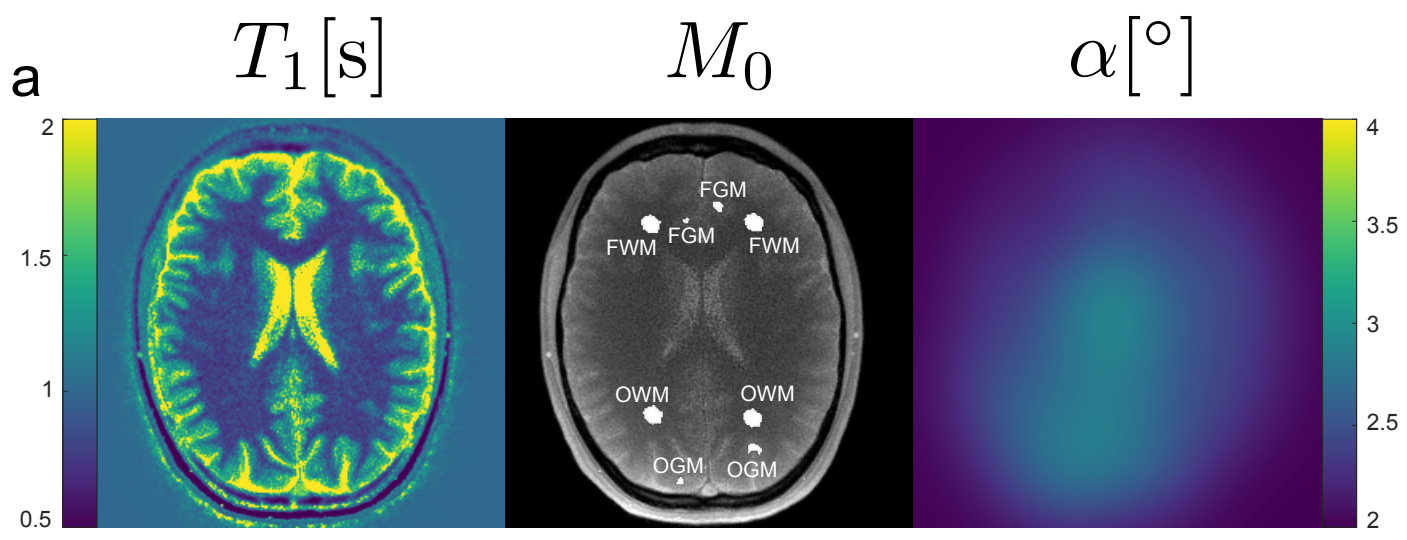

b

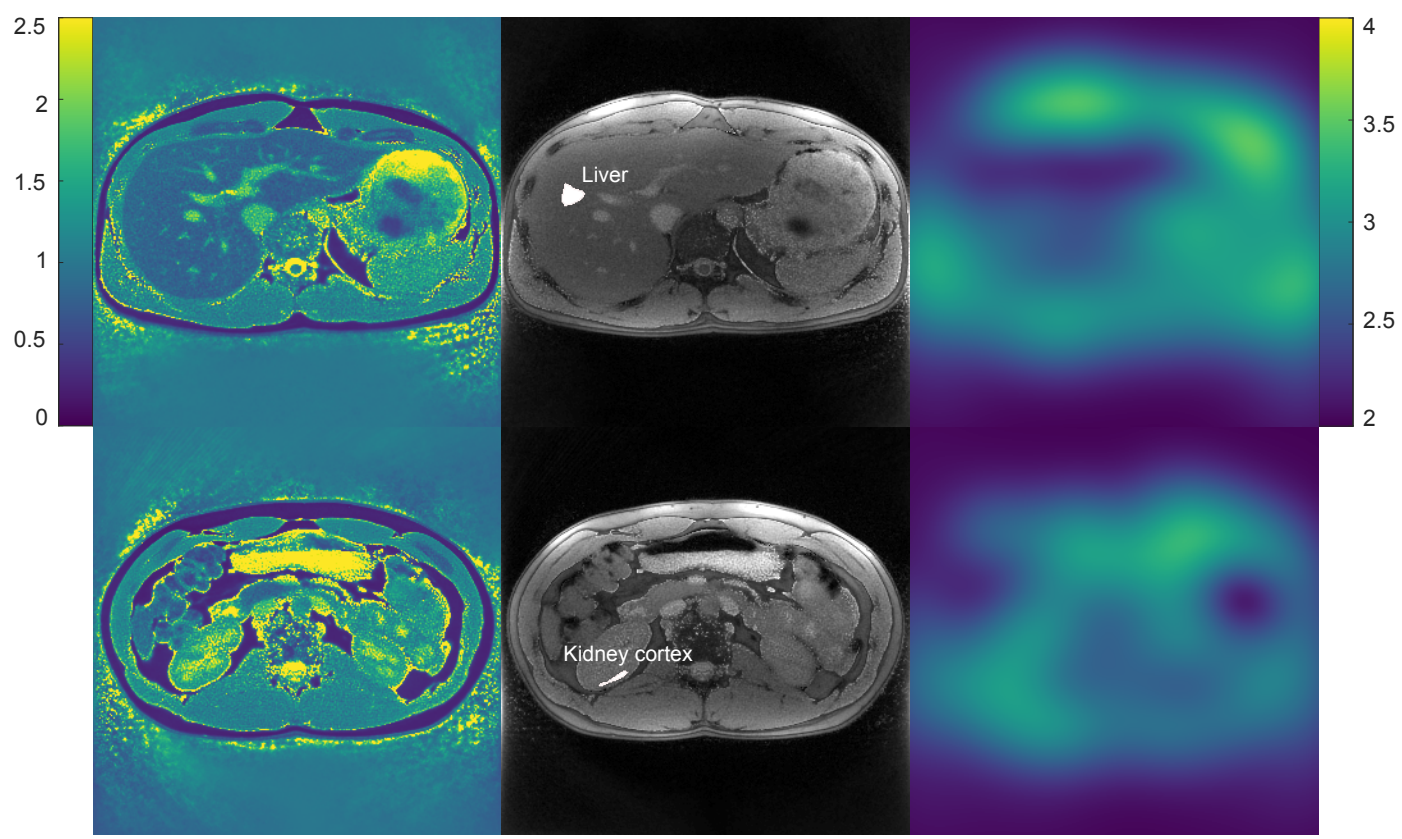

Figure 5.4: MODEL-BASEd $T_{1}$ MAPPING OF (a) A TRANSVERSE SECTION OF THE HUMAN BRAIN $\left(0.75 \times 0.75 \times 4 \mathrm{~mm}^{3}, 11\right.$ ITERATIONS) AND (b) TWO ABDOMINAL SECTIONS $\left(1.0 \times 1.0 \times 5 \mathrm{~mm}^{3}, 11\right.$ ITERATIONS). Each reconstruction used 71 frames $\times 21$ projections per frame $=1491$ radial projections. For ROI analyses see table 5.2. 


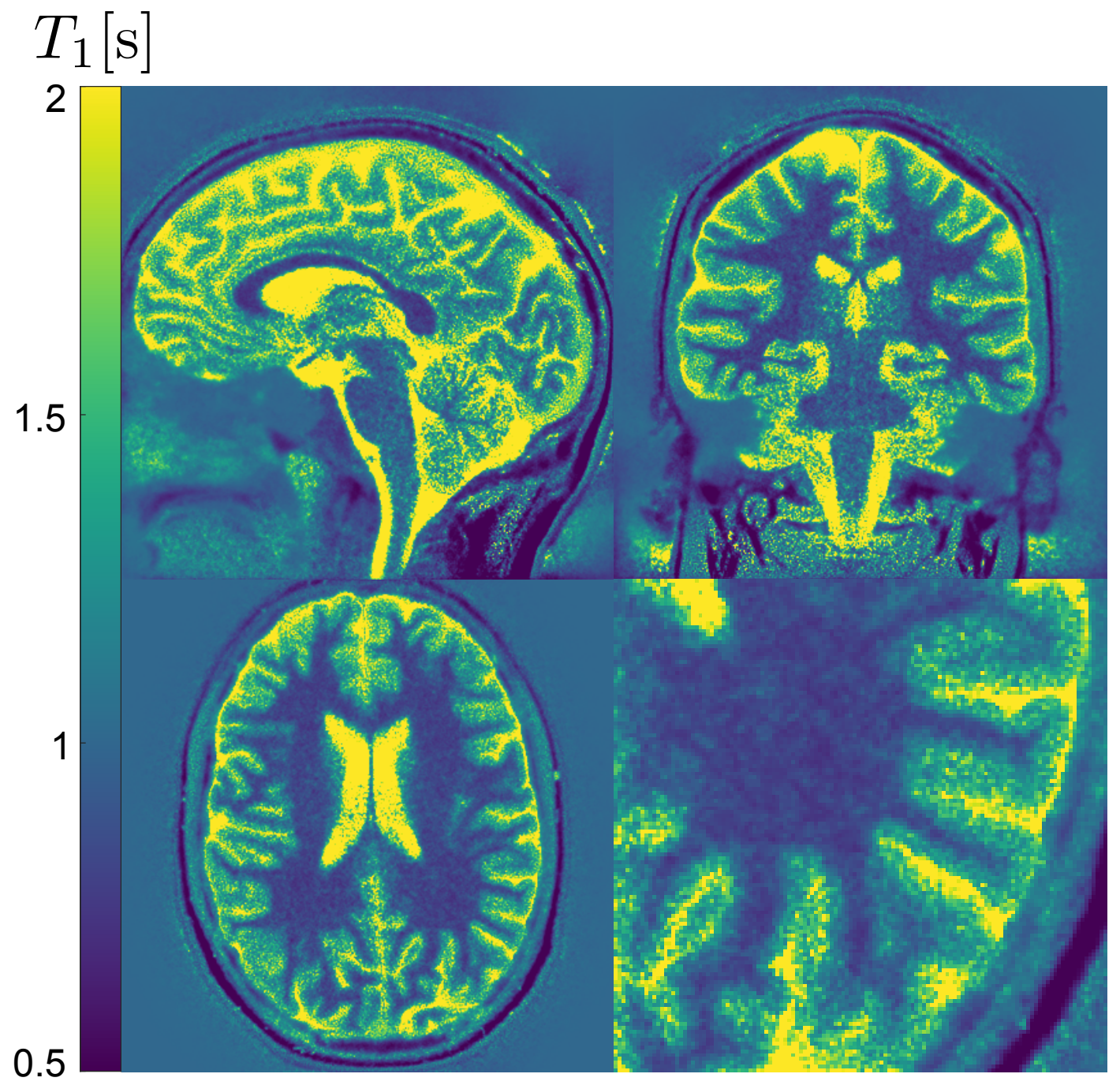

Figure 5.5: Model-BASEd $T_{1}$ MAPPING OF (UPPER LEFT) A SAgITTAL, (UPPER RIGHT) CORONAL AND (LOWER LEFT) TRANSVERSAL SECTION OF THE HUMAN BRAIN AT $0.5 \times 0.5 \times 4 \mathrm{~mm}^{3}$ RESOLUTION (12 ITERATIONS). A 3 -fold magnified view of the transversal section is shown in the lower right. Each reconstruction used 71 frames $\times 21$ projections per frame $=1491$ radial projections. 


\section{$5 \cdot 4$ DISCUSSION}

The proposed method describes the reconstruction of $T_{1}$, spin-density, and flipangle maps from a radial IR-FLASH experiment as a nonlinear inverse problem. It is demonstrated that the IRGNM is an appropriate solver for this problem as it not only allows for the incorporation of prior knowledge in form of different regularization terms, but also provides a solution at moderate computational cost as all occurring operators can be parallelized to a large degree for the effective use of graphical processing units.

The excellent temporal fidelity is an inherent advantage of a model-based reconstruction. It is unnecessary to bin data measured at different time points to single frames because no images are reconstructed directly. However, reconstruction can considerably be accelerated when this paradigm is weakened, as long as the temporal grid is fine enough to capture changes on the relevant time scales. Here, a maximum temporal grid size of $70 \mathrm{~ms}$ was determined by simulation and allowed a considerable speed-up in reconstruction time.

The presented method involves two crucial considerations. The first one is the choice of a specific regularization for each parameter map. The low spatial frequency assumption as a strong preconditioner only holds for the $R_{1}^{\prime}$ map. Moreover, the chosen regularization term for the $R_{1}$ map favors - for high regularization strengths - Laplacian solutions with smooth $R_{1}$ distributions [77] and does not penalize large $R_{1}$ values inappropriately as for the case of $L^{2}$ penalization on the $R_{1}$ map directly. The second point is the suppression of noise outside the radial sampling disc, which is implemented with a penalty on respective spatial frequencies. This effectively suppresses checkerboard-like artifacts that would otherwise spoil the resulting $T_{1}$ and spin-density maps.

Model-based reconstruction approaches are known to be sensitive to model mismatches, i.e., voxels with signal intensities that are in conflict with the underlying model [20, 32]. Chemical shift effects, imperfect spoiling, motion, or in-flow phenomena are not modeled by the Look-Locker signal and are a potential source of error. Unlike conventional reconstruction of image series, model-based reconstructions are prone to single-pixel mismatches, because optimization becomes a nonlocal problem. This kind of model violation was a prominent phenomenon in the transverse brain section cutting through the ventricles. The continuous in-flow of unsaturated cerebrospinal fluid into the slice cannot be characterized by a monoexponential recovery curve and hence arbitrary $T_{1}$ values can be produced. The restriction of the algorithm to a physical range of $T_{1}$ values ensures that the magnitude of the gradient values remains limited and the entire image is optimized instead of single pixels only [32]. However, the restriction to boundary values may temporarily lead to plateau-like regions in the parameter maps in which the con- 
vergence behavior of the IRGNM is altered. In practice, clearance of these artifacts can be achieved by choosing a sufficiently large number of iterations.

The actual choice of a suitable number of iterations depends on various sequencerelated factors such as SNR, flip angle, spatial resolution etc. Also the range of $T_{1}$ values and the chosen regularization for the $R_{1}$ parameter map (determined by the balancing parameter $\beta$ in eq. (5.8)) play a role. In fig. $5.3 \mathrm{~b}$, for instance, a $T_{1}$ dependent convergence speed is noticeable, with slowest convergence rate for the shortest $T_{1}$ values (and corresponding highest $R_{1}$ ) that are affected most by the remaining $L^{2}$ regularization in the convolution kernel $K$ (eq. (5.8)). This empirical determination of the number of Gauss-Newton steps is closely connected to noniterative regularization methods where the magnitude of respective regularization parameters is often found empirically as no common techniques for automatic determination have been established so far.

Tran-Gia et al. recently presented a model-based approach to $T_{1}$ mapping [21] that also relies on a radial IR-FLASH experiment. They proposed an iterative algorithm that alternates between image space and k-space domain and tries to fill missing k-space points by performing pixel-wise model fits or following a dictionary matching approach similar to [18]. However, their achieved spatial resolution of $1.6 \times 1.6 \times 4.0 \mathrm{~mm}^{3}$ as demonstrated in a human brain at a total acquisition time of $6 \mathrm{~s}$ is relatively low compared to our $0.75 \times 0.75 \times 4.0 \mathrm{~mm}^{3}$ in $4.5 \mathrm{~s}$. This has several reasons. The first one may lie in the fact that the present work used a threefold shorter TR of about $3 \mathrm{~ms}$, which results in more projections per time and consequently more spatial information. The second reason lies in the reduced degrees of freedom of the underlying reconstruction problem, even when the same model (i.e., three independent parameter maps) is employed. In the proposed method the strong regularization of the $R_{1}^{\prime}$ map reduces the degrees of freedom for this particular map because it can be described by a few low-frequency components only. The third reason is due to the incorporation of multi-coil information. Instead of simply combining individual coil signals to a sign-dependent sum of squares signal, the present method takes full advantage of parallel imaging by modelling the full signal formation which includes sensitivity maps from all individual channels.

The combination of a fast radial MRI sequence with a reconstruction algorithm, which optimally exploits the redundancy of a known signal model, bears the potential of pushing the limits for spatial resolution even further. When a slightly lower SNR is acceptable, the method achieves an in-plane resolution of up to $0.5 \mathrm{~mm}$. In addition, the efficient use of raw data renders the proposed method a promising technique for dynamic $T_{1}$ mapping, where the temporal resolution of sequential maps is the limiting factor. However, further investigations are necessary to extend the single IR-FLASH acquisition towards continuous mapping.

In this work, the determination of coil sensitivity profiles was performed in a preparatory step by NLINV. However, as both the proposed model-based recon- 
struction and the NLINV reconstruction rely on the IRGNM, a fusion of the two techniques is the next logical step. The joint estimation of parameter maps and coil profiles might lead to improved reconstruction results because prior knowledge in form of the model helps to determine the coil profiles more accurately and makes most efficient use of the available data. Such a combination might also reduce residual intensity inhomogeneities in the $M_{0}$ maps (a well known artifact in parallel imaging), which are best visible in abdominal scans due to the presence of subcutaneous fat in close proximity to some receiver coil elements.

At this stage, the main limitation is the need for offline reconstruction and depending on the image matrix - the long computational time of up to two hours. Because the latter problem mainly results from GPU-sided memory restrictions, this could be overcome by a memory-efficient CUDA/C++ implementation of the proposed algorithm in combination with a compression of the raw data in the time domain. Initial studies using a compression by singular value decomposition similar to [78] are promising. With the availability of online reconstructions the method warrants extensive clinical trials.

\section{$5 \cdot 5$ APPENDIX}

\subsubsection{Optimization}

Splitting the general Tikhonov regularization matrix in eq. (5.6) into its parameterspecific terms yields

$$
f(x)=\left\|\begin{array}{c}
F(x)-y \\
\sqrt{\alpha_{m}} L_{1}\left(M_{\mathrm{SS}}-M_{\mathrm{SS}}^{\mathrm{ini}}\right) \\
\sqrt{\alpha_{m}} L_{2}\left(R_{1}-R_{1}^{\mathrm{ini}}\right) \\
\sqrt{\alpha_{m}} L_{3}\left(\hat{R}_{1}^{\prime}-R_{1}^{\prime, \text { ini }}\right)
\end{array}\right\|_{2}^{2},
$$

where $M_{\mathrm{SS}}^{\mathrm{ini}}=0, R_{1}^{\mathrm{ini}}=1 \mathrm{~s}^{-1}$, and $L_{3} R_{1}^{\prime \text {,ini }}=-1 / \mathrm{TR} \ln \cos 2^{\circ}$ are the three a priori estimates of the parameter maps. $L_{1}, L_{2}$ and $L_{3}$ are regularization matrices representing parameter-specific regularization terms, while $\hat{R}_{1}^{\prime}=L_{3}^{-1} R_{1}^{\prime}$ represents the transformed "readout" relaxation rate. Matrix $L_{1}$ is chosen to be the identity matrix $\left(L_{1}=\mathbb{1}\right)$ and thus yields $L^{2}$ regularization as conventionally used for noise suppression in the context of inverse problems.

Matrix $L_{2}$ is a "stacked" operator [79] consisting of the unity matrix and a discrete approximation of the first-order spatial derivative operator. However, the ac- 
tual implementation only requires the operator representing $L_{2}^{H} L_{2}$, which can be computed efficiently by pixel-wise convolution with the 9-point-stencil

$$
K(\beta)=\frac{1}{N}\left(\beta\left[\begin{array}{ccc}
1 & 1 & 1 \\
1 & -8 & 1 \\
1 & 1 & 1
\end{array}\right]+(1-\beta)\left[\begin{array}{ccc}
0 & 0 & 0 \\
0 & -16 & 0 \\
0 & 0 & 0
\end{array}\right]\right) .
$$

The normalization constant $N$ ensures that the convolution preserves the relative magnitude of the map $\left(\sum\left|K_{i j}\right|=1\right)$ and the parameter $\beta$ allows balancing the effect of $L^{2}$ regularization and first-order derivate smoothing. The first addend represents a discrete version of the Laplace operator (as the derivate of the first-order spatial derivative operator) and the second addend represents pure $L^{2}$ regularization. $L^{2}$ regularization gives strong preference to solutions with smaller $L^{2}$ norms, which translate into parameter maps with smaller $R_{1}$ values. This was a problem particularly for pixels with short $T_{1}$ (and corresponding high $R_{1}$ ) which could be alleviated successfully by a suitable tradeoff between these two regularization terms. All reconstructions in this work employed a value of $\beta=0.9$.

The preconditioning matrix $L_{3}=\mathcal{F} W$ is a product of a Fourier transform $\mathcal{F}$ and a diagonal weighting matrix $W$ which acts on the transformed variable $\hat{R}_{1}^{\prime}$ causing a Fourier weighting of the form $\left(1+880|k|^{2}\right)^{-32}$ and corresponds to the Sobolev norm in the original space as presented in [31] to ensure a proper smoothness of the flip angle map. The scaling constant of 880 and the exponent of -32 were found heuristically and match the smoothness of the body coil used for excitation.

In each of the Gauss-Newton iterations the objective function in eq. (5.6) is linearized by a first order Taylor series around the current estimate $x_{m}$. The new iterate is then given by $x_{m+1}=x_{m}+p_{m}$ where the update $p_{m}$ is found by solving the resulting linear subproblem

$$
\left(D F^{H} D F+\alpha_{m} L^{H} L\right) p_{m}=D F^{H}\left(y-F\left(x_{m}\right)\right)-\alpha_{m} L^{H} L\left(x_{m}-x^{\text {ini }}\right),
$$

where $D F$ denotes the Fréchet derivate of the forward operator $F$ and $D F^{H}$ its corresponding adjoint. Each of the subproblems in eq. (5.9) is of the form $A x=b$ and solved by the conjugate gradient method with the stopping criterion $\left\|A x_{\text {approx }}-b\right\| /\|b\|<0.01$. The IRGNM requires a starting point, which is chosen to coincide with the a priori estimate $x^{\text {ini }}=\left(M_{\mathrm{SS}}^{\text {ini }}, R_{1}^{\text {ini }}, R_{1}^{\hat{\prime}_{1}^{\text {,ini }}}\right)^{T}$.

The numerical implementation of forward operator, Fréchet derivate and its adjoint follows Uecker and coworkers [31, 8o]: Instead of performing a computationally expensive explicit gridding operation, this operation is replaced by a convolution with a point-spread function $[*]_{\mathrm{PSF}}$ reflecting a particular sampling trajectory [81]. This convolution is implemented without approximations by two fast Fourier transform operations on a twofold oversampled grid

$$
[*]_{\mathrm{PSF}}=\mathcal{F}^{-1} T_{\vec{k}, 2 \times \mathrm{FoV}} \mathcal{F},
$$


where $T_{\vec{k}, 2 \times \text { FoV }}$ is a diagonal point-spread function (PSF) operator given by simply gridding "ones" on the sampled trajectory to a Cartesian grid. Hence, this operator can be computed in a preparatory step ahead of the actual reconstruction. Although each frame needs a different operator $T_{\vec{k}, 2 \times \text { FoV }}$ due to the unique reordering given by the Golden Angle scheme, all radial sampling patterns have in common that the edges of a Cartesian k-space (outside the radial sampling disc) are never sampled. When iteratively solving the nonlinear problem, these regions off the circular sampling disc may be dominated by strongly amplified noise, which deteriorates the $T_{1}$ map by checkerboard-like artifacts. A similar phenomenon has been reported for non-Cartesian SENSE reconstructions, where a k-space filter is introduced as a final step in image reconstruction [30] to suppress noisy data from k-space regions where calculations are highly ill-conditioned. Here, this idea is adapted by modifying the aforementioned operator $T_{\vec{k}, 2 \times \mathrm{FoV}}$ in such a way that spatial frequencies off the radial sampling disc are penalized:

$$
\hat{T}_{\vec{k}, 2 \times \mathrm{FoV}}=T_{\vec{k}, 2 \times \mathrm{FoV}}+\alpha W(|k|),
$$

where $W(|k|)=\frac{1}{2}-\frac{1}{\pi} \tan ^{-1}\left(\beta \frac{k_{c}-|k|}{k_{c}}\right)$ is the inverse $\mathrm{k}$-space filter with cut-off frequency $k_{c}=\frac{1}{2} k_{\max }$ and $\beta=100$ as used in [30], and $\alpha$ the mean value of $T_{\vec{k}, 2 \times \text { FoV }}$. This modification effectively suppresses the undesired artifacts and comes at no additional cost in the reconstruction algorithm.

\subsubsection{Scaling}

In this implementation, the raw data vector $y$ was normalized with respect to its squared $L^{2}$ norm summed over the available coils $\left(\sum_{j}\left|y_{j}\right|^{2}=10^{5}\right)$ and the PSF operator $\hat{T}_{\vec{k}, 2 \times \mathrm{FoV}}$ was normalized with respect to its sum of squared magnitude values in time domain $\left(\sum_{t}\left|\hat{T}_{t, k=0}\right|^{2}=1\right)$. Normalization of the vector comprising all coil sensitivity profiles $C=\left(C_{1}, C_{2}, \ldots, C_{N}\right)^{T}$ was performed similarly with respect to the pixel of highest absolute sensitivity $\left(\sum_{j} \max _{x, y}\left|C_{j}(x, y)\right|^{2}=10^{-3}\right)$. 

DYNAMIC $T_{1}$ MAPPING IN FIRST-PASS DCE-PERFUSION STUDIES

Design and setup of the experiment presented in this chapter was done in close collaboration with Jost Michael Kollmeier. Jost joined our group as a master student and performed his master thesis under my supervision. His thesis is entitled "Perfusion Phantom Studies using Real-Time Magnetic Resonance Imaging" [82] and deals with the application of existing real-time MR protocols to monitor contrast agent induced signal changes.

\section{I INTRODUCTION}

In dynamic contrast-enhanced (DCE) MRI, a high-concentration contrast agent (CA) is rapidly injected into the cardiovascular system. The resulting CA bolus serves as a tracer and travels with the blood stream to the tissue of interest where it induces changes in the MR signal intensity (first pass). These intensity changes are spatially resolved by fast imaging techniques and - in a post-processing step - converted into changes in contrast agent concentration. Knowledge about the concentration time curve in the capillary bed, the so-called arterial input function (AIF), and the application of kinetic analyses yield parametric maps reflecting not only information about the anatomy but also function. Therefore, DCE MRI has become a valuable tool in many clinical applications including evaluation of kidney, heart, breast, prostate, and brain (for current reviews see [83-87]).

Despite the simplicity of the underlying principle, quantitative DCE imaging is a technically challenging method in which both, the generation of data suitable for quantitative measurements of CA kinetics and the extraction of quantitative parameters from fitting of pharmacokinetic models to this data, are prone to infringe of requirements and are still subject of active research. A suitable data generation relies on an acquisition protocol that addresses several (often conflicting) requirements, among them (i) sensitivity to CA and access to CA concentration, (ii) high temporal resolution, and (iii) high spatial resolution.

One of the requirements that is most difficult to achieve is the access to CA concentration. As long as a linear relationship between signal intensity and CA concentration is assumed [88-90], an absolute determination of concentration is not necessary. However, at higher CA concentration this assumption often breaks as saturation introduces nonlinear effects. In this case, the measured signal intensities have to be converted to CA concentrations, a transformation prone to errors. An 
alternative acquisition strategy is to acquire quantitative $T_{1}$ maps at every time point in the DCE experiment. Because the main drawback of this method is the tremendous decrease in sampling speed when combined with conventional $T_{1}$ mapping techniques, an echo planar imaging (EPI)-based readout [91] is normally employed. This imaging technique allows for acquisition times below $3 \mathrm{~s}$, but similar to many other EPI-based methods, a pronounced susceptibility to artifacts such as ghosting, signal distortion, and loss in regions with short $T_{2}^{*}$.

Here, a new model-based $T_{1}$ mapping technique is employed that relies on radial IR FLASH readout in combination with the advanced reconstruction algorithm developed in chapter 5. Similar short acquisition times as for EPI-based methods can be obtained due to the efficient use of raw data. As no gold standard technique has established so far, a first step in evaluating potential application of this new technique has to be done in vitro with availability of ground truth. The availability of ground truth also implies that the mimicked perfusion processes should be as simple as possible, i.e., complex multi-compartment situations should be avoided.

In this study, we demonstrate the general compliance of dynamic model-based $T_{1}$ mapping with first-pass perfusion experiments. In particular, a commercially available two-compartment perfusion phantom is adapted and utilized to mimic perfusion similar to in vivo situations. A gadolinium based CA is used as a tracer and concentration time curves are obtained by sequential model-based reconstruction of $T_{1}$ maps. The simple and most common parametric Tofts model (TM) [92] is used for quantitative analysis.

\subsection{METHODS}

\subsubsection{Perfusion Phantom and Experimental Setup}

Simulation of in vitro blood flow and tracer kinetics is performed by a commercially available multimodality DCE perfusion phantom (Shelly Industrial Automation Inc., Toronto, Canada) originally developed for the use with CT [93-95] but meanwhile also used in the context of MRI [96]. This phantom is shipped together with a peristaltic pump (Masterflex L/S with pump head model 77250-62, ColeParmer, Illinois, USA), two turbine flow meters (FLR 1000, Omega Engineering, Connecticut, USA), two needle valves, an injection port, and a diverter valve (see fig. 6.1). For rapid injection of a contrast medium a programmable power injector with microprocessor control of flow rate, volume, and timing (Angiomat 6000, Liebel-Flarsheim, Cincinnati, USA) was provided by the local university hospital. The vendor-supplied plastic bed for the tubing was replaced by a water environment consisting of a customized water-filled PET bottle. The setup of these components is outlined schematically in fig. 6.2. The entire circulation system is split into two parts: Pump, flow meter, valves, and power injector contain ferromagnetic 
materials and must be operated from outside the RF chamber, while the perfusion phantom together with the water bed is placed inside the scanner's bore. For a full DCE walk-through, demineralized water from the container is pumped into the upstream connection of the injection port, gets mixed with the CA provided by the power injector, and finally enters as a bolus of CA the RF chamber. To ensure a sufficient polarization inside the static magnetic field, the bolus travels a loop of about $1 \mathrm{~m}$ tubing length inside the bore of the scanner before passing the imaging slice. From here, the tubing leads to the phantom and back, with both phantom outputs passing through the slice again. Flow meter and valves outside the RF chamber allow to control the ratio of flow in the two phantom outputs. Finally, the position of the diverter valve determines whether the system is run in open or closed circuit mode (open circuit mode is necessary as soon as CA has entered the system to avoid recirculation effects). Power injector and pump start/stop are operated manually at this stage.

The actual tissue response curves are generated inside the cylinder (fig. 6.Ib,c): CA enters the phantom via the distribution tube and distributes through its perforated surface into the cylinder compartment. This mimics blood supply and perfusion of tissue with an arterial input (phantom input), venous output (distribution tube output) and the tissue of interest (cylinder output).

\subsubsection{Pharmacokinetic Model}

When quantifying CA uptake in tissue, it is import to choose the correct pharmacokinetic model that accurately describes the underlying perfusion process. This is normally achieved by modeling the CA diffusion processes in the tissue of interest by compartmental models. Complex blood-tissue exchanges of CA are described as a collection of interacting components, called compartments. Two assumptions are therefore enough to completely define the CA kinetics [97]: All compartments are assumed to be homogeneous (i.e., well mixed) and the CA fluxes between them are related to their corresponding concentrations. Rate equations are stated by exploiting the law of tracer mass conservation and finally solved to yield the desired kinetics.

Figure 6.3 shows a schematic illustration of CA transfer in vivo and in the phantom. For the in vivo situation, CA is assumed to diffuse to and from the extravascular extracellular space (EES) whereas the extravascular intracellular space (EIS) does not participate in the exchange processes. Hence, in the respective twocompartment model the tissue is modelled as one single compartment, while the concentration in the intravascular space (IVS) is considered to be the input to the system. The transfer rates involved in this model are commonly labeled $K_{\text {trans }}$ and $K_{\text {trans }} / v_{e}$ [98], where $v_{e}$ is the volume of EES per unit volume of tissue, i.e., the fractional volume. For quantification, two concentrations are sampled over time: The 

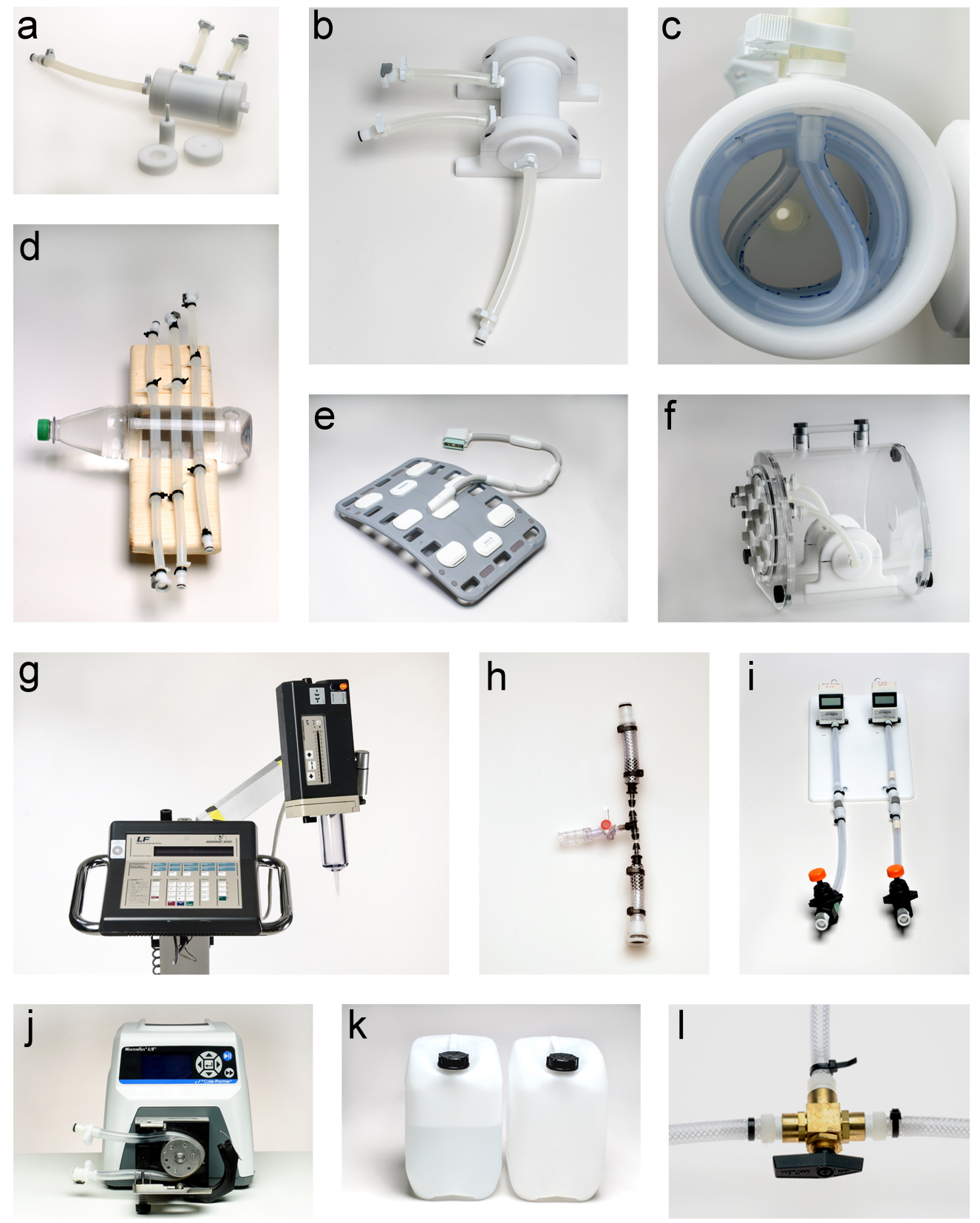

Figure 6.1: PhOtOgRAPHS OF ALL PARTS IN THE FIRST-PASS PERfUSION EXPERIMENT. Setup and function are described in section 6.2.1. (a) exchange cylinder with volume reduction modules, (b) exchange cylinder with stand, (c) spiraling, perforated distribution tube, (d) tubing inside PET water bottle, (e) 18-channel body array coil, (f) torso shell for CT measurements, (g) power head and keyboard console of Power Injector, $(\mathrm{h})$ injection port, (i) needle valves and turbine flow meters, (j) peristaltic pump, (k) $10 \mathrm{~L}$ water and waste containers, (1) 3-way ball diverter.

Photos: Irene Böttcher-Gajewski, MPI-BPC 2016 


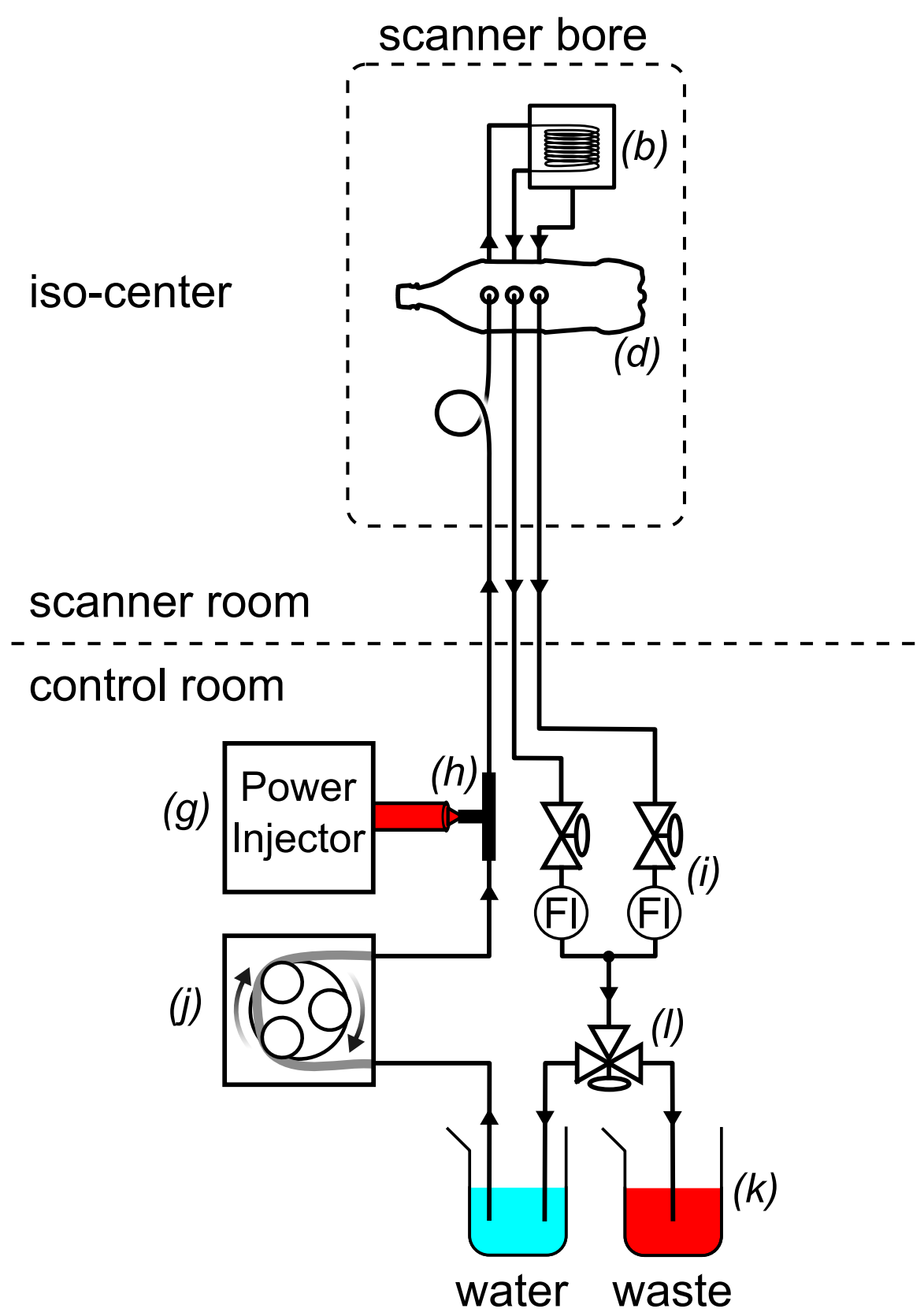

Figure 6.2: SCHEMATIC SETUP OF THE IN VITRO FIRST-PASS PERFUSION EXPERIMENT. The entire circulation system is split into two parts. Pump, flow meter, valves, and power injector are operated from the control room, the perfusion phantom and the water environment are placed in the bore of the MR scanner. See fig. 6.1 for details on components. As soon as CA has entered the system, the diverter valve (l) must be flipped from "water" to "waste" to avoid recirculation.

(Scheme created in collaboration with Jost Kollmeier) 
tracer concentration in the IVS or plasma compartment $C_{p}$ and the concentration in the perfused voxel of tissue $C_{t}$. The latter quantity is either interpreted as the concentration in the EES $\left(C_{t}=v_{e} C_{e}\right)$ by neglecting the contribution of the IVS or as the weighted concentration including the IVS $\left(C_{t}=v_{e} C_{e}+v_{p} C_{p}\right)$. The former interpretation is known as TM [92] and valid for example in weakly vascularized tissue, whereas the latter is commonly called extended Tofts model (ETM) [99] and includes the fractional plasma volume $v_{p}$. Following Khalifa et al. [97], these two pharmacokinetic models are considered the best-established and most straightforward models for interpreting $T_{1}$-weighted DCE-MR images.

The CA transfer process in the phantom differs. Here, no backflow into the distribution tube is possible. An additional outflow with rate $K_{\text {trans }}$ enables the CA to leave the cylinder and provides the possibility to measure the corresponding tracer concentration without contribution from the emulated plasma compartment. Changes in the fractional volume of the EES can be simulated by scaling of the obtained response curves accordingly and intravascular contributions can be simulated by mixing the measured signal $C_{p}$ with the obtained response before fitting. The setup is therefore flexible and allows simulation of transfer processes as described by the TM or ETM with controllable pharmacokinetic parameters $K_{\text {trans }}, v_{e}$, and $v_{p}$.

Although the compartmental models for tissue and phantom differ in structure, the same mathematical solution is found. For the TM, the tissue concentration is given according to [98] by

$$
C_{t}(t)=K_{\text {trans }} \int_{0}^{t} C_{p}\left(t^{\prime}\right) e^{-\left(K_{\text {trans }} / v_{e}\right)\left(t-t^{\prime}\right)} d t^{\prime},
$$

which represents a convolution of the AIF with a tissue impulse response function. For the phantom, $v_{e}$ can be set to 1 when $C_{t}(t)$ is the tracer concentration in the cylinder (compartment $e$ ) is measured and eq. (6.1) can then be written as

$$
C_{t}(t)=K_{\text {trans }} C_{p}(t) * e^{-K_{\text {trans }} t},
$$

where $(*)$ denotes temporal convolution. A temporal shift between $C_{t}(t)$ and $C_{p}(t)$ - as automatically introduced when measuring at physically different positions can be accounted for by introducing a shift constant $t_{0}$ in the convolution kernel:

$$
C_{t}(t)=K_{\text {trans }} C_{p}(t) * e^{-K_{\text {trans }}\left(t-t_{0}\right)}
$$

\subsubsection{Contrast Agent}

Gadobutrol was used as a CA ("Gadovist" $1.0 \mathrm{~mol} \mathrm{~mL}^{-1}$, Schering AG, Germany) and diluted to $0.01 \mathrm{~mol} \mathrm{~mL}^{-1}$ with purified water before injection. Longitudinal 
TISSUE

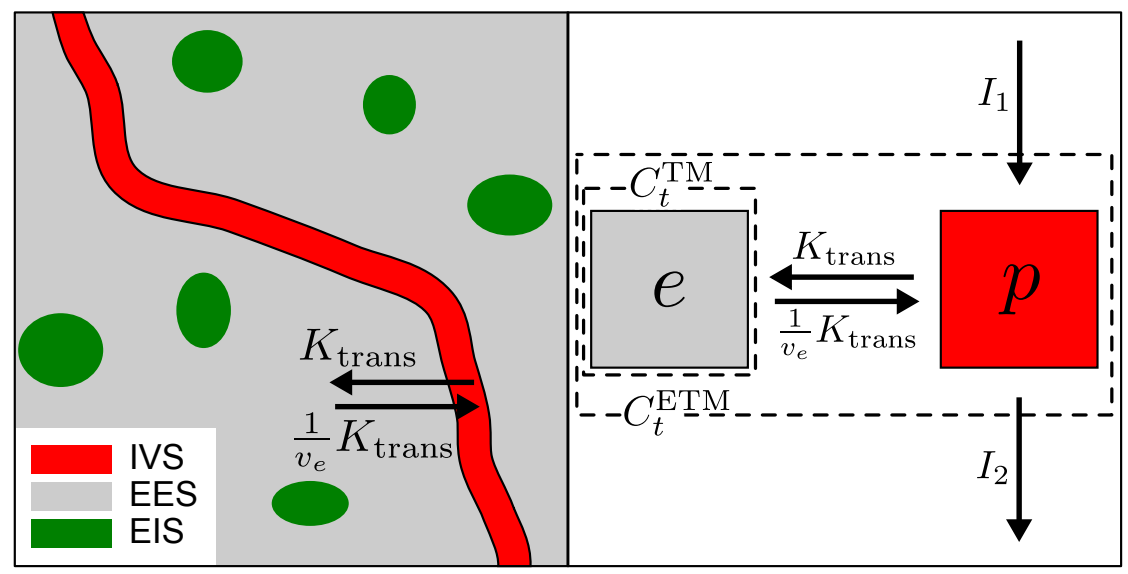

PHANTOM

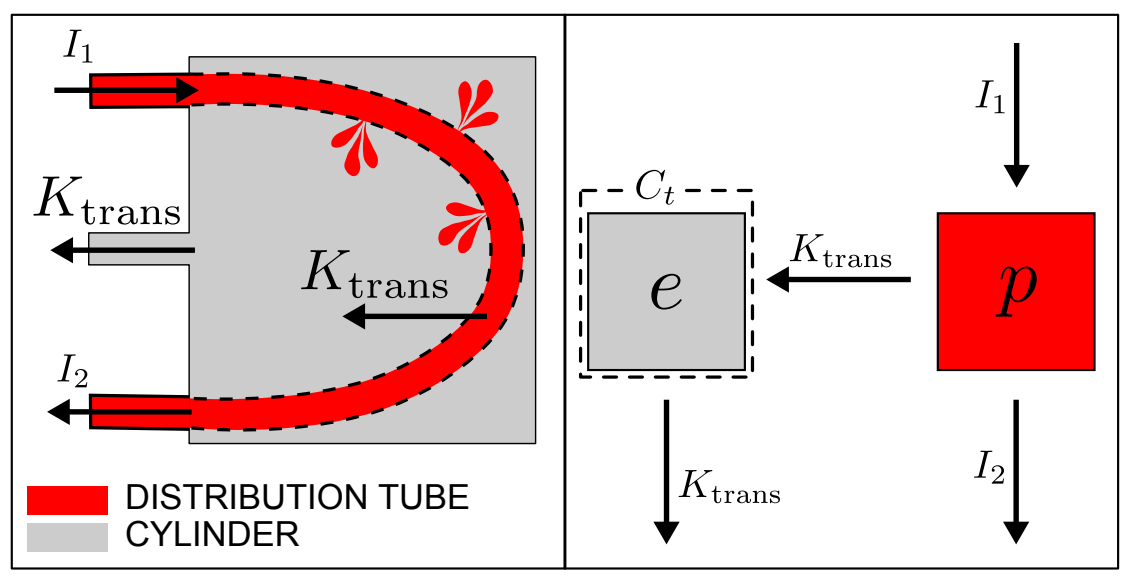

Figure 6.3: SCHEMATIC ILLUSTRATION OF THE ASSUMED CONTRAST AGENT TRANSFER IN THE tissue (TOP ROW) AND IN THE PHANTOM (вотtOM ROW). Tissue: The CA diffuses from the intravascular space (IVS) to the extravascular extracellular space (EES) with rate $K_{\text {trans }}$ and returns with $k_{\text {ep. }}$. The extravascular intracellular space (EIS) is assumed to be free of CA. Phantom: The backflow from compartment $e$ to $p$ is omitted and replaced by an additional outflow rate equal to $K_{\text {trans. The rates }} I_{1}, I_{2}$, and $K_{\text {trans }}$ can be controlled by the overall flow rate and the outflow ratio. 
and transverse relaxivities were determined via a dilution series at $3 \mathrm{~T}$ (for details see [82]) to $r_{1}=(4.6 \pm 0.1) \mathrm{L} \mathrm{mmol}^{-1} \mathrm{~s}^{-1}$ and $r_{2}=(5.4 \pm 0.1) \mathrm{L} \mathrm{mmol}^{-1} \mathrm{~s}^{-1}$. The concentration dependence of $R_{1}$ is assumed to be linear [100, 101] and concentration changes are related to time-variant relaxation rates by

$$
R_{1}(t)=R_{10}+r_{1} C(t),
$$

where $R_{10}$ denotes the native relaxation rate as observed in absence of CA. This linear relationship implicitly assumes that the exchange of water molecules between tissue compartments is infinitely fast [102].

\subsubsection{Protocols and Reconstruction}

Imaging was performed at $3 \mathrm{~T}$ field strength using an MRI system with $80 \mathrm{mTm}^{-1}$ gradients (Magnetom Prisma, Siemens Healthcare, Erlangen, Germany) and the 18channel thorax coil in combination with suitable segments of the 32-channel spine coil. To avoid any in-flow effects when quantifying $T_{1}$, the first-pass perfusion experiment was performed under pulsed flow. For this purpose the OFF-time of the pump was fixed to $10 \mathrm{~s}$ and the ON-time to $10 \mathrm{~s}$, or $5 \mathrm{~s}$ respectively, resulting in duty cycle values of $50 \%$, or $33 \%$. $T_{1}$ mapping was performed by a Locker-Locker type acquisition employing an IR radial FLASH sequence in combination with a newly developed model-based reconstruction technique (chapter 5 ). To enable $T_{1}$ mapping synchronous to the pump cycle periods of $20 \mathrm{~s}$ (or $15 \mathrm{~s}$, respectively), the original sequence was modified to include an arbitrary dead-time before each inversion pulse. This dead-time gives control over the data acquisition periods and allows - if chosen sufficiently long - full recovery of magnetization prior to each inversion pulse. After manually synchronizing start of pump and data acquisition, CA was injected with completion of the first ON/OFF pump cycle. The outflow ratio of the phantom was controlled via the flow meters and set to $1 / 2$, resulting in absolute flow values of $300 \mathrm{~mL} \mathrm{~min}^{-1}$ in the input tube and $150 \mathrm{~mL} \mathrm{~min}^{-1}$ in both cylinder output and distribution tube output. Table 6.1 summarizes further measurement and reconstruction parameters of the perfusion experiment.

Expected $T_{1}$ values of lower than $200 \mathrm{~ms}$ are challenging to reconstruct accurately for two reasons: First, the corresponding high relaxation rates lead to fast signal changes on a short time scale. This precludes to usage of large binning sizes and consequently makes high demands on memory consumption. Second, a Tikhonov type regularization on a parameter map containing these high rates most likely leads to an underestimation of the rates, as higher rates are quadratically penalized. To cope with these challenges the original reconstruction algorithm (chapter 5) was modified. In this study, the number of projections per frame (binning size) was reduced to 13 which results in a temporal grid of $32.5 \mathrm{~ms}$. The higher demands in memory were compensated by reducing the number of principle components to 4 


\begin{tabular}{ll}
\hline Parameter & Value \\
\hline Flow and Injection & \\
Pump flow rate & $300 \mathrm{~mL} \mathrm{~min}^{-1}$ \\
Pump duty cycle & $50 \%, 33 \%$ \\
Outflow ratio $F_{\text {cyl }} / F_{\text {pump }}$ & $1 / 2$ \\
Injection concentration & $10 \mathrm{mmol} \mathrm{L}-1$ \\
Injection bolus volume & $9.8 \mathrm{~mL}, 9.9 \mathrm{~mL}$ \\
Injection duration & $10 \mathrm{~s}$ \\
& \\
Sequence & $320 \times 320 \mathrm{~mm}^{2}$ \\
FoV & $320 \times 320$ \\
Matrix size & $1.0 \mathrm{~mm}$ \\
In-plane resolution & $5 \mathrm{~mm}$ \\
Slice thickness & $2.5 \mathrm{~ms}$ \\
TR & $1.62 \mathrm{~ms}$ \\
TE & $1420 \mathrm{~Hz} /$ pixel \\
Bandwidth & $4^{\circ}$ \\
Flip angle & $68.75^{\circ}(\mathrm{small} \mathrm{Golden} \mathrm{Angle)}$ \\
Angular displacement & $4 \mathrm{~s}$ \\
Acquisition time & $16 \mathrm{~s}, 11 \mathrm{~s}$ \\
per $T_{1}$ map & 32,64 \\
Dead-time & \\
Total number of $T_{1}$ maps & \\
Reconstruction & 4 \\
Virtual channels & $13 \times 92=1196$ \\
Number of projections & \\
used per $T_{1}$ map & 13 projections \\
Binning size & \\
Gauss-Newton iterations & 11 \\
\hline
\end{tabular}

Table 6.1: INJeCTION, SEQUENCE, AND RECONSTRUCTION PROTOCOLS USED IN THE PERFUSION EXPERIMENT. When two values specified, the latter value refers to the second experiment.

${ }^{1}$ Nominal injection bolus volume was set to $10 \mathrm{~mL}$, actual volume deviates depending on achieved flow. 
and restricting the total number of projections per $T_{1}$ map to $13 \times 92=1196$. The regularization term of the $R_{1}$ map within the reconstruction algorithm was modified by shifting the weight in the "stacked" operator away from pure $L^{2}$ regularization towards the part that represents the Laplacian operator. An adaptation of the corresponding 9-point-stencil $K(\alpha)$ by heuristically choosing a value of $\alpha=0.99$.

All $T_{1}$ maps were reconstructed individually using the same starting values as in the original algorithm (see chapter 5 ). This waiver of temporal regularization explicitly precludes any "crosstalk" effects or temporal blurring between individual $T_{1}$ maps.

\subsubsection{Quantitative Analysis}

Quantitative analysis was performed by ROI averaging of the obtained $R_{1}$ values, baseline subtraction, and conversion to concentration time curves using eq. (6.4). The AIF was obtained from concentration time curves as measured in either the input tube or the distribution tube output and the cylinder output was treated as the tissue response $C_{t}$. The quantities $K_{\text {trans }}$ and $t_{0}$ were then determined by a twoparameter nonlinear least-squares fit of eq. (6.3) to the measured data. Convolution of the AIF with the tissue response function was formulated as a matrix equation in which the convolution matrix elements were calculated according to [103, eq. 14].

The obtained quantity $K_{\text {trans }}$ is related to the absolute volume of the cylinder compartment by

$$
V_{\text {cyl }}=\frac{F_{\text {cyl }}}{K_{\text {trans }}}=\frac{F_{\text {pump }} R}{K_{\text {trans }}},
$$

where $F_{\text {cyl }}$ and $F_{\text {pump }}$ are the flow rate in the cylinder compartment and the overall flow rate given by the pump, and $R=F_{\text {cyl }} / F_{\text {pump }}$ is the outflow ratio.

\subsection{RESULTS}

Figure 6.4 shows $T_{1}$ maps for 5 exemplary time points out of 64 as obtained after offline model-based reconstruction of the $5 \mathrm{~s}$ sampling experiment. Here and hereafter the denoted time refers to the elapsed time of the kinetic process, which is stopped when the pump stops. The passage of CA through the input and output tubes lead to shortening in $T_{1}$ down to $160 \mathrm{~ms}$. The corresponding tissue response follows with a delay and recovers slowly to baseline. All maps are artifact-free, in particular no streak artifacts as known from radial undersampling are visible. ROI-wise averaging yields $R_{1}$ as a function of time for all three compartments.

The corresponding time courses are depicted in fig. 6.5 for the $10 \mathrm{~s}$ (fig. 6.5a,c) and $5 \mathrm{~s}$ sampling experiment (fig. $6.5 \mathrm{~b}, \mathrm{~d}$ ). Shaded bounds indicate standard deviations as obtained after ROI averaging. The integrated amount of tracer is calculated by trapezoidal numerical integration of the concentration time curves and multipli- 


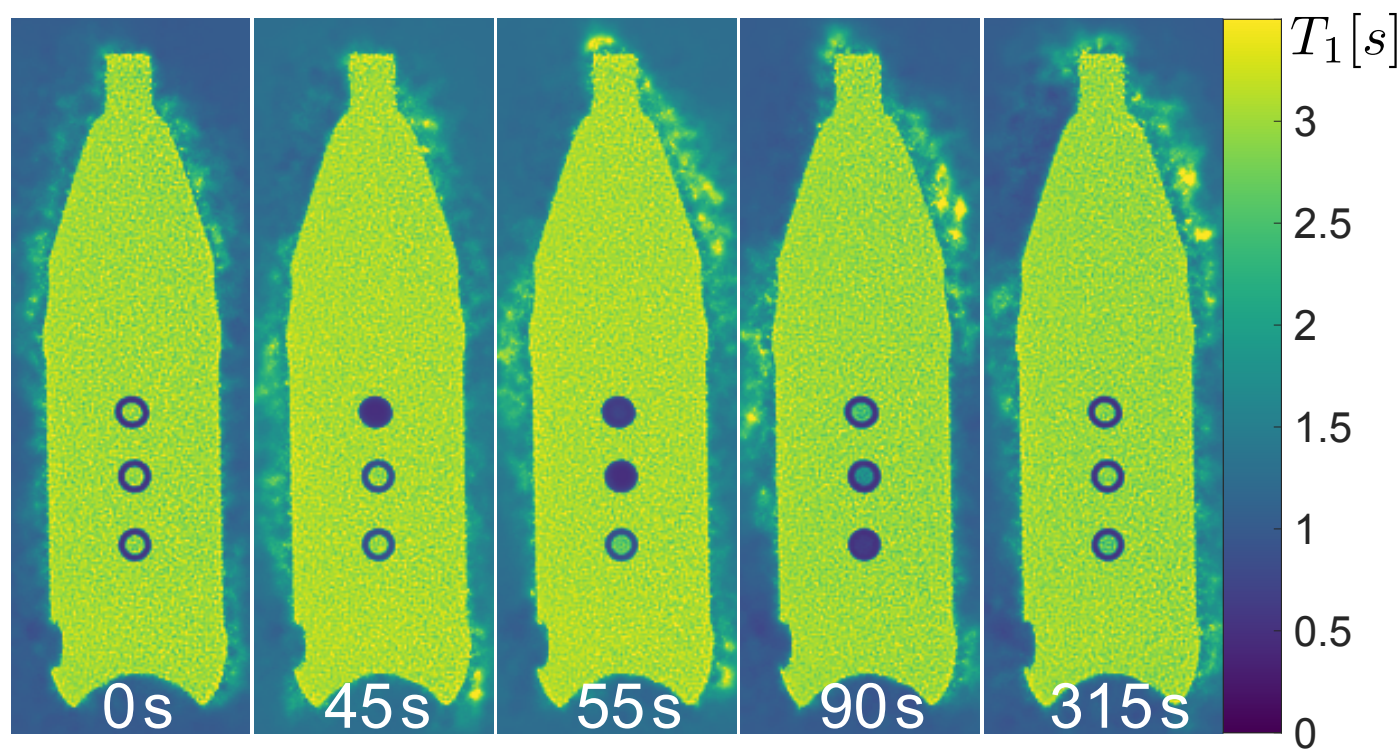

Figure 6.4: $T_{1}$ MAPS AFTER MODEL-BASED RECONSTRUCTION EXEMPLARILY SHOWN FOR 5 TIME POINTS. Slice is oriented perpendicular to the tubing (top to bottom: phantom input, distribution tube output, and cylinder output). Maps were acquired before injection of CA $(0 \mathrm{~s})$, at peak in input tube $(45 \mathrm{~s})$, at peak in distribution tube (55s), at peak in cylinder output (90s), and at end of wash-out (315s). All maps are rotated and cropped for visualization purposes.

cation by the corresponding flow rates. For quantitative analyzes of tracer kinetics the AIF is either taken from tracer concentrations in the input tube (blue) or in the distribution tube output (red) and the tissue response is taken from the concentrations measured in the cylinder output (yellow). Fitting of the convolution kernel in eq. (6.3) to this data yields the time shift constant $t_{0}$ and the exchange rate $K_{\text {trans. }}$. The fitted tissue responses (dashed black) are plotted on top of the measured tissue responses (AIF from input tube). The $5 \mathrm{~s}$ sampling experiment yields larger maximum $R_{1}$ rates in the phantom input and distribution tube output and similar tissue responses when compared to the $10 \mathrm{~s}$ sampling experiment. The amount of tracer integrated over the entire time course and summed over the two outputs matches the input, however, the true amount of tracer as calculated from injection volume and concentration is slightly smaller $(0.98 \mathrm{mmol}$ and $0.99 \mathrm{mmol}$, respectively).

Results of the two-parameter fit for both experiments can be found in table 6.2. Values for the calculated cylinder volumes $V_{\text {cyl }}$ are precise, but inaccurate as they underestimate the true volume by about $13 \%$ (10 s sampling interval) and $6 \%$ ( $5 \mathrm{~s}$ sampling interval). Significant differences between taking the AIF from phantom input or cylinder output can only be found for the time shift constant $t_{0}$, where the cylinder output shows a smaller $t_{0}$ due to smaller tubing length differences (see setup in section 6.2.1). 
a

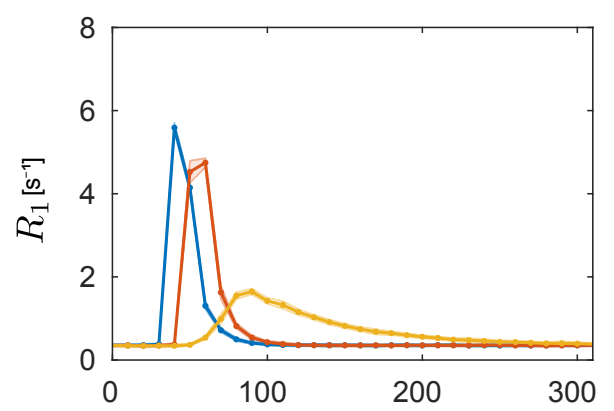

C

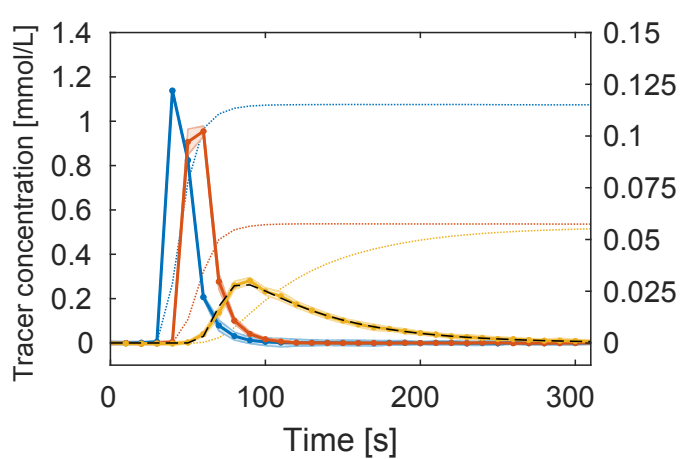

b

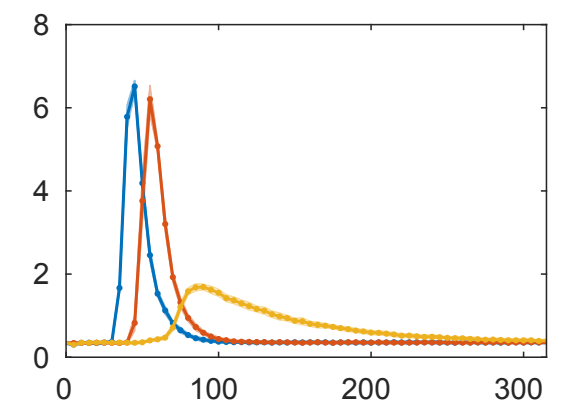

d

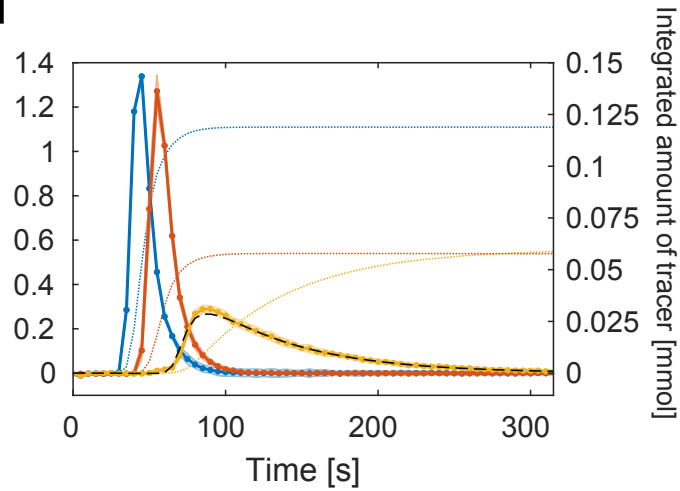

Figure 6.5: LONGITUDINAL RELAXATION RATES AND CALCULATED TRACER CONCENTRATIONS AS A FUNCTION OF TIME. $R_{1}$ maps were evaluated ROI-wise (blue: phantom input, red: distribution tube output, yellow: cylinder output) for the $10 \mathrm{~s}$ sampling experiment (a) and for the $5 \mathrm{~s}$ sampling experiment (b). After baseline subtraction, tracer concentration can be calculated with known relaxivity according to eq. (6.4) for both sampling rates $(\mathrm{c}, \mathrm{d})$. Dashed black lines represent the fitted tissue responses when taking the AIF from the phantom input tube and assuming the exponential impulse response function in eq. (6.3). Dotted lines indicate integrated amount of tracer substance assuming constant and known flow. 


\begin{tabular}{lllll}
\hline AIF & $t_{0}[\mathrm{~s}]$ & $K_{\text {trans }}\left[\mathrm{min}^{-1}\right]$ & calc. $V_{\text {cyl }}[\mathrm{ml}]$ & true $V_{\text {cyl }}[\mathrm{ml}]$ \\
\hline $\begin{array}{l}\text { Sampling interval 10 s } \\
\text { phantom input }\end{array}$ & $23.4 \pm 0.7$ & $1.05 \pm 0.02$ & $144 \pm 3$ & 165 \\
$\quad$ distribution tube output & $12.0 \pm 0.6$ & $1.05 \pm 0.02$ & $143 \pm 3$ & 165 \\
& & & & \\
$\begin{array}{l}\text { Sampling interval 5s } \\
\text { phantom input }\end{array}$ & $27.5 \pm 0.5$ & $0.96 \pm 0.01$ & $156 \pm 2$ & 165 \\
$\quad$ distribution tube output & $16.9 \pm 0.5$ & $0.97 \pm 0.01$ & $155 \pm 2$ & 165 \\
\hline
\end{tabular}

Table 6.2: QUANTITATIVE RESUlTS OF THE PERFUSION EXPERIMENT. AIF from either phantom input or distribution tube output and tissue response from cylinder output was fitted by eq. (6.3). Initial values were $K_{\text {trans }}^{\text {ini }}=2 \mathrm{~min}^{-1}, t_{0}^{\text {ini }}=0$ if AIF taken from distribution tube output and $t_{0}^{\mathrm{ini}}=30 \mathrm{~s}$ else. The cylinder volume $V_{\text {cyl }}$ was calculated according to eq. (6.5).

\subsection{DISCUSSION}

The presented experimental setup allows to produce concentration time curves as known from first-pass DCE experiments under controlled conditions. The circulation system was integrated into our MR system and can be fully controlled from outside the RF chamber. Operating the pump in pulsed mode allows to measure without in-flow effects by virtually "freezing" the CA dynamics. As this option does not exist for in vivo applications, it is a unique tool for investigating the influence of flow and temporal resolution on the quantitative results.

Dynamic $T_{1}$ mapping was used to measure concentration time curves without taking the detour via conversion of signal intensities into CA concentrations. The newly developed model-based reconstruction technique (see chapter 5) was able to reconstruct artifact-free high resolution $T_{1}$ maps from data acquired in $4 \mathrm{~s}$ including $T_{1}$ values as short as $160 \mathrm{~ms}$. However, the necessary small binning size of 13 projections per time point had to be compensated by a decreased number of virtual channels in comparison to the original work. This limitation may be overcome in future by a memory efficient implementation of the algorithm in combination with a smart compression of the raw data along the time direction.

The recovery periods between consecutive $T_{1}$ maps of at least $11 \mathrm{~s}$ were synchronized with the OFF-times of the pump. Of course, dynamic $T_{1}$ mapping in vivo requires a significant shortening of this period. An acceleration could be achieved by replacing the initial inversion pulse with a saturation module similar to [104] rendering the waiting period superfluous. However, further investigations are required to assess the reduced SNR and the changes necessary to apply a model-based reconstruction to this modified preparation strategy.

Data evaluation employed parametric deconvolution of the measured tissue response curves with an exponential kernel as given by the TM. The corresponding 
fits described the measured data accurately indicating that the exchange processes in the hardware perfusion phantom can be described by the corresponding twocompartment TM. The quantitative analyzes also revealed that the calculated total amount of injected tracer substance at phantom input matches the output. However, the true amount - as given by bolus volume and concentration used - is slightly overestimated. The reason might lie in the fact that an increase in CA concentration also leads to an increase in $R_{2}$ (and consequently $R_{2}^{*}$ ) as the CA also has a transverse relaxivity. However, the presence of larger $R_{2}$ values reduces the inversion efficiency of the initial adiabatic pulse due to increased transverse relaxation during the pulse $[105,106]$. This imperfect inversion influences the calculated $R_{1}$ values because the starting value of the recovery curve is assumed to be the negative equilibrium magnetization in the signal model. A corresponding underestimation of its magnitude results in an overestimation of the calculated $R_{1}$ values and tracer concentrations, consequently.

The presented data further shows that a sampling interval of $10 \mathrm{~s}$ was too long to correctly reflect the rapid concentration changes in the AIF (as produced by the chosen protocol). However, a sampling interval of $5 \mathrm{~s}$ also leads to a slight underestimation of the measured cylinder volume, which might be explained by the fact, that the estimated value refers to a volume of a compartment that is at any time well-mixed. Although the cylindrical shaped exchange compartment and the spiral alignment of the distribution tube favors mixing, this assumption might break, especially when rapid changes in CA concentration take place. As a consequence, the effective compartment volume could be smaller.

The experimental setup offers a huge variety of simulation scenarios. The specific compilation of injection, MR sequence, and reconstruction protocol presented here, is only an exemplary choice demonstrating general feasibility. The same setup is for instance also used to investigate the abilities of already established real-time MRI sequences in the context of quantitative DCE perfusion [82]. It might become a valuable tool for further investigations of sequential $T_{1}$ mapping techniques, $T_{1}$ mapping approaches under in-flow conditions, and reconstruction approaches attempting to directly model the obtained tissue responses. Full potential is probably released with protocols as close as possible to a specific clinical application and simulated pharmacokinetic parameter matching physiological conditions as observed in vivo. 
In this thesis, four different aspects of quantitative MRI techniques are addressed: Data acquisition, data reconstruction, signal modeling, and experimental validation.

The first part focuses on a problem well known from gradient-echo MR sequences with short TR: Spoiling strategies have to be employed that prevent the build up of coherent transverse magnetizations. Commonly, these spoiling schemes are realized by additional gradient events possibly in combination with modified RF phases $[37,42,107]$. In real-time MRI, these additional events lead to prolonged TR, reduced frame rates, and, inevitably, result in a loss of temporal resolution. In this thesis, a spoiling method for radial FLASH sequences was developed that avoids this loss by entirely relying on manipulation of the transmit and receive phases. Furthermore, a characteristic connection between the employed sampling pattern and the observed artifact pattern was demonstrated in numerical simulation and in vitro measurements when no additional spoiling measures are taken. The spoiling efficiency of the proposed method was demonstrated in in vivo measurements of the human brain for different sampling schemes. This development effectively removed undesired artifacts at no additional costs and thus paved the way towards successful application of alternative trajectories as used in the presented model-based $T_{1}$ mapping method. Moreover, all real-time sequences in our group now employ this spoiling scheme as a default. In future, based on the published method, it might be possible to find a combination of a sampling scheme and a particular RF phase value sequence that results in superior spoiling performance. However, further investigations are needed together with a deeper understanding about the complex interplay of acquired phase, spatial position, off-resonance, slice profile, and voxel averaging effects.

Chapter 4 addresses the need for a signal model that describes the magnetization time course in single-shot multi-slice IR FLASH experiments. The developed analytic expression describes the signal time course correctly and expands the single-slice Deichmann correction [24] on multi-slice $T_{1}$ mapping scenarios. Simulations and in vitro phantom studies confirmed its validity and in [25], a co-authored publication, this model was successfully used for pixelwise fitting and $T_{1}$ quantification in human brain and abdomen based on nonlinear reconstruction of image time series. The presented derivation might also help to develop a signal model for the case of myocardial $T_{1}$ mapping, where even for single-slice imaging, different relaxation regimes exist due to strong through-plane movements of the myocardial muscle. 
In chapter 5 , a model-based reconstruction technique was presented for $T_{1}$ mapping from single-shot IR radial FLASH acquisitions. Here, a joint reconstruction of the $T_{1}$, spin-density, and flip-angle map is formulated as a nonlinear inverse problem and solved by the IRGNM. The increased computational demands could be mitigated by outsourcing of highly parallelizable code modules to the GPU. Precision and accuracy of the method was evaluated in numerical simulations (with ground truth available by design) and in vitro measurements (with comparison to gold standard measurements). The proposed method makes efficient use of raw data as (i) parameter maps are reconstructed directly without attempting to calculate images and (ii) prior knowledge on each parameter map was included by parameter-specific regularization terms. Two reconstruction-related problems, namely the build up of checkerboard artifacts and the occurrence of model violation by negative $T_{1}$ values, were successfully solved by simple yet effective means: A k-space filter adapted from spiral imaging methods [30] was integrated into the gridding operator and suppressed noise from regions outside the sampling disc at no additional computational cost. A restriction to positive $T_{1}$ values, which renders the reconstruction a bound-constraint problem, was realized by checking each calculated iterate for feasibility and possibly falling back on predefined boundary values. The optimal tradeoff between impaired temporal fidelity and increased reconstruction speed was found by simulation. For this purpose, the influence of the number of projections assigned to the same time point (i.e., the binning size) was investigated with respect to quantitative accuracy. For a proper quantification of $T_{1}$ values larger than $300 \mathrm{~ms}$ a maximal temporal grid size of $70 \mathrm{~ms}$ was determined. $T_{1}$ mapping abilities of the novel reconstruction method were demonstrated in the human brain and in abdominal sections at in-plane resolutions of up to $0.5 \times 0.5 \mathrm{~mm}^{2}$ with a total acquisition time of $3.6 \mathrm{~s}$ to $5 \mathrm{~s}$. The obtained $T_{1}$ values are in agreement with literature data obtained at $3 \mathrm{~T}$. This work was submitted as a manuscript to "NMR in Biomedicine" (in revision at the time of thesis submission).

In the last chapter, both the developed spoiling scheme and the model-based reconstruction technique are applied to the field of DCE MRI. An in vitro flow cycle was set up to mimic tissue perfusion with a commercially available first-pass perfusion phantom. This experimental setup allows to simulate a variety of injection and acquisition protocols as well as an evaluation of different reconstruction techniques. In an exemplary study, the model-based reconstruction technique was used to quantify the influence of the temporal resolution on the calculated exchange rates. Operating the pump in a pulsed mode allowed to virtually "freeze" the CA dynamics, which precluded any in-flow effects during data acquisition. The full control over compartment volumes, injection and flow rates, and acquisition protocols renders this setup an excellent benchmark tool for the evaluation of future MR methods in quantitative first-pass DCE MRI. 
In summary, a new time-efficient spoiling strategy for real-time MRI acquisitions was developed and evaluated, a novel signal model for multi-slice $T_{1}$ mapping was derived theoretically, a model-based reconstruction algorithm for single-shot $T_{1}$ mapping was designed and implemented, and a first-pass DCE phantom was set up and used for quantitative perfusion analysis. 



\section{BIBLIOGR A PHY}

[1] J. Frahm, A. Haase, D. Matthaei, W. Hänicke, and K.-D. Merboldt. Verfahren und Vorrichtung zur Aufnahme von Spinresonanzdaten. Patent. DE3504734. 1985 (cit. on p. 1).

[2] A. Haase, J. Frahm, D. Matthaei, W. Hänicke, and K.-D. Merboldt. "FLASH imaging. Rapid NMR imaging using low flip-angle pulses". Journal of Magnetic Resonance (1969) 67.2 (1986), pp. 258-266. DOI: 10.1016/0022-2364 (86) 90433-6 (cit. on p. 1).

[3] J. Hennig, A. Nauerth, and H. Friedburg. "RARE imaging: a fast imaging method for clinical MR". Magnetic Resonance in Medicine 3.6 (1986), pp. 823833. DOI: $10.1002 / \mathrm{mrm} .1910030602$ (cit. on p. 1).

[4] S. Zhang. "Real-time magnetic resonance imaging". PhD thesis. http : // hdl . handle . net/11858/00-1735-0000-000D-F258-8: University of Göttingen, 2009 (cit. on p. 1).

[5] M. Uecker, S. Zhang, and J. Frahm. "Nonlinear inverse reconstruction for real-time MRI of the human heart using undersampled radial FLASH". Magnetic Resonance in Medicine 63.6 (2010), pp. 1456-1462. DoI: 10.1002 / mrm. 22453 (cit. on p. 1).

[6] M. Uecker, S. Zhang, D. Voit, A. Karaus, K.-D. Merboldt, and J. Frahm. "Real-time MRI at a resolution of $20 \mathrm{~ms}$ ". NMR in Biomedicine 23.8 (2010), pp. 986-994. DOI: 10.1002/nbm. 1585 (cit. on pp. 1, 17, 22, 34, 50).

[7] S. Zhang, K. T. Block, and J. Frahm. "Magnetic resonance imaging in real time: Advances using radial FLASH". Journal of Magnetic Resonance Imaging 31.1 (2010), pp. 101-109. DOI: 10.1002/jmri.21987 (cit. on pp. 1, 18).

[8] P. W. Iltis, J. Frahm, D. Voit, A. A. Joseph, E. Schoonderwaldt, and E. Altenmüller. "High-speed real-time magnetic resonance imaging of fast tongue movements in elite horn players". Quantitative Imaging in Medicine and Surgery 5.3 (2015), pp. 374-381. DOI: 10.3978/j. issn.2223-4292.2015.03. 02 (cit. on pp. 1, 17, 22).

[9] A. A. Joseph, K.-D. Merboldt, D. Voit, S. Zhang, M. Uecker, J. Lotz, and J. Frahm. "Real-time phase-contrast MRI of cardiovascular blood flow using undersampled radial fast low-angle shot and nonlinear inverse reconstruction". NMR in Biomedicine 25.7 (2012), pp. 917-924. DOI: 10.1002/nbm. 1812 (cit. on pp. 1, 50). 
[10] S. Zhang, A. Olthoff, and J. Frahm. "Real-time magnetic resonance imaging of normal swallowing". Journal of Magnetic Resonance Imaging 35.6 (2012), pp. 1372-1379. DOI: 10.1002/jmri.23591 (cit. on p. 1).

[11] D. Voit, S. Zhang, C. Unterberg-Buchwald, J. M. Sohns, J. Lotz, and J. Frahm. "Real-time cardiovascular magnetic resonance at $1.5 \mathrm{~T}$ using balanced SSFP and 40 ms resolution". Journal of Cardiovascular Magnetic Resonance 15 (2013), p. 79. DOI: 10.1186/1532-429X-15-79 (cit. on p. 1).

[12] A. Niebergall, S. Zhang, E. Kunay, G. Keydana, M. Job, M. Uecker, and J. Frahm. "Real-time MRI of speaking at a resolution of 33 ms: Undersampled radial FLASH with nonlinear inverse reconstruction". Magnetic Resonance in Medicine 69.2 (2013), pp. 477-485. Dor: 10.1002/mrm. 24276 (cit. on pp. 1, 50).

[13] A. Joseph, J. T. Kowallick, K.-D. Merboldt, D. Voit, S. Schaetz, S. Zhang, J. M. Sohns, J. Lotz, and J. Frahm. "Real-time flow MRI of the aorta at a resolution of $40 \mathrm{msec}^{\prime \prime}$. Journal of Magnetic Resonance Imaging 40.1 (2014), pp. 206-213. DOI: 10.1002/jmri.24328 (cit. on p. 1).

[14] M. Fasshauer, A. A. Joseph, J. T. Kowallick, C. Unterberg-Buchwald, K. D. Merboldt, D. Voit, M. Steinmetz, W. Staab, S. Schaetz, S. Zhang, J. Frahm, J. Lotz, and J. M. Sohns. "Real-time phase-contrast flow MRI of haemodynamic changes in the ascending aorta and superior vena cava during Mueller manoeuvre". Clinical Radiology 69.10 (2014), pp. 1066-1071. DOI: 10.1016/j.crad.2014.06.004 (cit. on p. 1).

[15] S. Zhang, A. A. Joseph, D. Voit, S. Schaetz, K.-D. Merboldt, C. UnterbergBuchwald, A. Hennemuth, J. Lotz, and J. Frahm. "Real-time magnetic resonance imaging of cardiac function and flow-recent progress". Quantitative Imaging in Medicine and Surgery 4.5 (2014), pp. 313-329. DOI: $10.3978 / \mathrm{j}$. issn.2223-4292.2014.06.03 (cit. on p. 1).

[16] S. Dreha-Kulaczewski, A. a. Joseph, K.-D. Merboldt, H.-C. Ludwig, J. Gärtner, and J. Frahm. "Inspiration Is the Major Regulator of Human CSF Flow". Journal of Neuroscience 35.6 (2015), pp. 2485-2491. DOI: 10.1523/ JNEUROSCI . 3246-14. 2015 (cit. on p. 1).

[17] K. T. Block, M. Uecker, and J. Frahm. "Undersampled radial MRI with multiple coils. Iterative image reconstruction using a total variation constraint". Magnetic Resonance in Medicine 57.6 (2007), pp. 1086-1098. DOI: 10.1002/mrm. 21236 (cit. on p. 2).

[18] M. Doneva, P. Börnert, H. Eggers, C. Stehning, J. Sénégas, and A. Mertins. "Compressed sensing reconstruction for magnetic resonance parameter mapping". Magnetic Resonance in Medicine 64.4 (2010), pp. 1114-1120. DoI: 10 . 1002/mrm. 22483 (cit. on pp. 2, 8, 45, 47, 58). 
[19] T. J. Sumpf, M. Uecker, S. Boretius, and J. Frahm. "Model-based nonlinear inverse reconstruction for T2 mapping using highly undersampled spinecho MRI". Journal of Magnetic Resonance Imaging 34.2 (2011), pp. 420-428. DOI: 10.1002/jmri.22634 (cit. on pp. 2, 8, 45, 48).

[20] J. Tran-Gia, D. Stäb, T. Wech, D. Hahn, and H. Köstler. “Model-based Acceleration of Parameter mapping (MAP) for saturation prepared radially acquired data". Magnetic Resonance in Medicine 70.1992 (2013), pp. 15241534. DOI: 10.1002/mrm. 24600 (cit. on pp. 2, 8, 45, 47, 57).

[21] J. Tran-Gia, T. Wech, T. Bley, and H. Köstler. "Model-Based Acceleration of Look-Locker T1 Mapping". Plos One 10.4 (2015), eo122611. Dor: $10.1371 /$ journal.pone. 0122611 (cit. on pp. 2, 8, 45, 58).

[22] F. Knoll, J. G. Raya, R. O. Halloran, S. Baete, E. Sigmund, R. Bammer, T. Block, R. Otazo, and D. K. Sodickson. "A model-based reconstruction for undersampled radial spin-echo DTI with variational penalties on the diffusion tensor". NMR in Biomedicine 28.3 (2015), pp. 353-366. Dor: 10.1002/ nbm.3258 (cit. on pp. 2, 8, 45).

[23] Z. Tan, V. Roeloffs, D. Voit, A. A. Joseph, M. Untenberger, K. D. Merboldt, and J. Frahm. "Model-based reconstruction for real-time phase-contrast flow MRI: Improved spatiotemporal accuracy". Magnetic Resonance in Medicine (2016). DOI: 10.1002/mrm. 26192 (cit. on pp. 2, 8, 45, 48, 96).

[24] R. Deichmann and A. Haase. "Quantification of T1 values by SNAPSHOTFLASH NMR imaging". Journal of Magnetic Resonance (1969) 96.3 (1992), pp. 6o8-612. DoI: 10.1016/0022-2364 (92) 90347-A (cit. on pp. 3, 19, 27, 28, $33,40,45,46,48,77)$.

[25] X. Wang, V. Roeloffs, K. D. Merboldt, D. Voit, and J. Frahm. “Single-shot Multi-slice T1 Mapping at High Spatial Resolution - Inversion-Recovery FLASH with Radial Undersampling and Iterative Reconstruction". Open Medical Imaging Journal (2015), pp. 1-8 (cit. on pp. 3, 27, 30, 34, 50, 77, 96).

[26] S. Winkelmann, T. Schaeffter, T. Koehler, H. Eggers, and O. Doessel. "An optimal radial profile order based on the golden ratio for time-resolved MRI". IEEE Transactions on Medical Imaging 26.1 (2007), pp. 68-76. DOI: 10.1109/TMI. 2006.885337 (cit. on pp. 3, 18, 48, 50).

[27] E. M. Haacke, R. W. Brown, M. R. Thompson, and R. Venkatesan. Magnetic Resonance Imaging - Physical Principles and Sequence Design. 6. 1999, p. 914 (cit. on p. 6).

[28] M. H. Levitt. spin dynamics: Basics of Nuclear Magnetic Resonance. John Wiley \& Sons, 2001 (cit. on p. 6). 
[29] P. C. Lauterbur. "Image Formation by Induced Local Interactions: Examples Employing Nuclear Magnetic Resonance". Nature 242 (1973), pp. 190-191. DOI: 10.1038/242190a0 (cit. on pp. 7, 18).

[30] K. P. Pruessmann, M. Weiger, P. Börnert, and P. Boesiger. "Advances in sensitivity encoding with arbitrary k-space trajectories". Magnetic Resonance in Medicine 46.4 (2001), pp. 638-651. DoI: 10.1002/mrm. 1241 (cit. on pp. 8, $61,78)$.

[31] M. Uecker, T. Hohage, K. T. Block, and J. Frahm. "Image reconstruction by regularized nonlinear inversion-Joint estimation of coil sensitivities and image content". Magnetic Resonance in Medicine 60.3 (2008), pp. 674-682. DoI: 10.1002/mrm. 21691 (cit. on pp. 8, 34, 6o).

[32] T. J. Sumpf, A. Petrovic, M. Uecker, F. Knoll, and J. Frahm. "Fast T2 mapping with improved accuracy using undersampled spin-echo MRI and model-based reconstructions with a generating function". IEEE Transactions on Medical Imaging 33.12 (2014), pp. 2213-2222. DOI: 10 . 1109/TMI . 2014. 2333370. arXiv: 1405.3574 (cit. on pp. 8, 45, 48, 57).

[33] K. Block, M. Uecker, and J. Frahm. "Model-Based Iterative Reconstruction for Radial Fast Spin-Echo MRI". IEEE Transactions on Medical Imaging 28.11 (2009), pp. 1759-1769. DOI: 10.1109/TMI.2009. 2023119 (cit. on pp. 8, 45).

[34] B. P. Sutton. "Physics Based Iterative Reconstruction for MRI : Compensating and Estimating Field Inhomogeneity and Relaxation by c" (2003) (cit. on p. 8).

[35] J. Frahm, W. Hanicke, and K. D. Merboldt. "Transverse Coherence in Rapid FLASH NMR Imaging". Journal of Magnetic Resonance (1969) 72.2 (1987), pp. 307-314. DOI: 10.1016/0022-2364 (87)90292-7 (cit. on p. 10).

[36] A. P. Crawley and R. M. Henkelman. "Errors in T2 estimation using multislice multiple-echo imaging". Magn Reson Med 4.1 (1987), pp. 34-47. Dor: 10.3109/15360288.2011.573528 (cit. on p. 10).

[37] Y. Zur, M. L. Wood, and L. J. Neuringer. "Spoiling of transverse magnetization in steady-state sequences". Magnetic Resonance in Medicine 21.2 (1991), pp. 251-263. DOI: 10.1002/mrm. 1910210210 (cit. on pp. 10, 17, 18, 33, 77).

[38] L. E. Drain. "A Direct Method of Measuring Nuclear Spin-Lattice Relaxation Times". Proc. Phys. Soc. Lond. A 62 (1949), p. 301 (cit. on p. 11).

[39] E. L. Hahn. "An Accurate Nuclear Magnetic Resonance Method for Measuring Spin-Lattice Relaxation Times". Physical Review 76.1 (1949), pp. 145-146. DOI: 10.1103/PhysRev.76.145 (cit. on p. 11). 
[40] V. Roeloffs, D. Voit, and J. Frahm. "Spoiling without additional gradients: Radial FLASH MRI with randomized radiofrequency phases". Magnetic Resonance in Medicine (2015). DOI: 10.1002/mrm. 25809 (cit. on pp. 15, 33, 48, 96).

[41] A. P. A. Crawley, M. L. Wood, and R. M. Henkelman. "Elimination of transverse coherences in FLASH MRI". Magnetic Resonance in Medicine 8.3 (1988), pp. 248-26o. DOI: 10.1002/mrm. 1910080303 (cit. on pp. 17, 18, 23, 33).

[42] W. Lin and H. K. Song. "Improved signal spoiling in fast radial gradientecho imaging: Applied to accurate T1 mapping and flip angle correction". Magnetic Resonance in Medicine 62.5 (2009), pp. 1185-1194. DoI: 10.1002 / mrm. 22089 (cit. on pp. 17, 77).

[43] G. H. Glover and J. M. Pauly. "Projection reconstruction techniques for reduction of motion effects in MRI". Magnetic Resonance in Medicine 28.2 (1992), pp. 275-289. DOI: 10.1002/mrm. 1910280209 (cit. on p. 18).

[44] V. Rasche, R. W. De Boer, D. Holz, and R. Proksa. "Continuous radial data acquisition for dynamic MRI". Magnetic Resonance in Medicine 34.5 (1995), pp. 754-761. DOI: 10.1002/mrm. 1910340515 (cit. on p. 18).

[45] K. Scheffler and J. Hennig. "Reduced circular field-of-view imaging". Magnetic Resonance in Medicine 40.3 (1998), pp. 474-480. DOI: 10.1002 / mrm . 1910400319 (cit. on p. 18).

[46] B. Hargreaves. Bloch equation simulator. Downloaded from http: / / wwwmrsrl. stanford. edu/ brian/mritools .html, Published January 13, 2004. Updated October 24, 2005. Accessed October 14, 2014. (cit. on p. 19).

[47] P. Vakil, S. A. Ansari, M. C. Hurley, H. Bhat, H. H. Batjer, B. R. Bendok, C. S. Eddleman, and T. J. Carroll. "Magnetization spoiling in radial FLASH contrast-enhanced MR digital subtraction angiography". Journal of Magnetic Resonance Imaging 36.1 (2012), pp. 249-258. DOI: 10.1002/jmri.23630 (cit. on p. 23).

[48] A. Brau and G. Johnson. "The impact of view order on steady-state magnetization in radial sampling". In: Proceedings of the 11th Annual Meeting of the ISMRM, Toronto, Canada. 2003, p. 209 (cit. on p. 23).

[49] R. Kaiser, E. Bartholdi, and R. Ernst. "Diffusion and field-gradient effects in NMR Fourier spectroscopy". The Journal of Chemical Physics 60.8 (1974), pp. 2966-2979 (cit. on p. 23).

[50] J. Hennig. "Multiecho imaging sequences with low refocusing flip angles". Journal of Magnetic Resonance (1969) 78.3 (1988), pp. 397-407 (cit. on p. 23). 
[51] H.-L. Margaret Cheng, N. Stikov, N. R. Ghugre, and G. A. Wright. "Practical medical applications of quantitative MR relaxometry". Journal of Magnetic Resonance Imaging 36.4 (2012), pp. 805-824 (cit. on p. 27).

[52] R. Kaptein, K. Dijkstra, and C. Tarr. "A single-scan fourier transform method for measuring spin-lattice relaxation times". Journal of Magnetic Resonance (1969) 24.2 (1976), pp. 295-300. DOI: 10.1016/0022-2364(76)90039-1 (cit. on pp. 27,45$)$.

[53] J. Tran-Gia, T. Wech, D. Hahn, T. A. Bley, and H. Köstler. “Consideration of slice profiles in inversion recovery Look-Locker relaxation parameter mapping". Magnetic Resonance Imaging 32.8 (2014), pp. 1021-1030. DOI: 10.1016/j.mri.2014.05.012 (cit. on p. 29).

[54] G. E. Santyr, E. J. Fairbanks, F. Kelcz, and J. A. Sorenson. “Off-resonance spin locking for MR imaging". Magnetic Resonance in Medicine 32.1 (1994), pp. 43-51. DOI: 10.1002/mrm. 1910320107 (cit. on p. 31).

[55] D. C. Look and D. R. Locker. "Time Saving in Measurement of NMR and EPR Relaxation Times". Review of Scientific Instruments 41.2 (1970), pp. 250251. DOI: 10.1063/1.1684482 (cit. on pp. 33, 45, 48).

[56] F. Huang, S. Vijayakumar, Y. Li, S. Hertel, and G. R. Duensing. "A software channel compression technique for faster reconstruction with many channels". Magnetic Resonance Imaging 26.1 (2008), pp. 133-141. Dor: 10.1016/j . mri.2007.04.010 (cit. on pp. 34, 50).

[57] G. Ries. "Eddy current transients and forces in cryostat walls of superconducting solenoids". IEEE Transactions on Magnetics 24.1 (1988), pp. 516-519. DOI: $10.1109 / 20.43970$ (cit. on p. 34).

[58] P. Jehenson, M. Westphal, and N. Schuff. "Analytical method for the compensation of eddy-current effects induced by pulsed magnetic field gradients in NMR systems". Journal of Magnetic Resonance (1969) 90.2 (1990), pp. 264-278. DOI: 10.1016/0022-2364 (90)90133-T (cit. on p. 34).

[59] E. A. Badea and O. Craiu. "Eddy Current Effects in MRI Superconducting Magnets". IEEE Transactions on Magnetics 33.2 (1997), pp. 1330-1333 (cit. on p. 34).

[6o] K. Block and M. Uecker. "Simple method for adaptive gradient-delay compensation in radial MRI". In: Proceedings of the 19th International Society for Magnetic Resonance Imaging. 2011, p. 2816 (cit. on pp. 34, 50).

[61] G. Liberman, Y. Louzoun, and D. Ben Bashat. "T1 mapping using variable flip angle SPGR data with flip angle correction". Journal of Magnetic Resonance Imaging 40.1 (2014), pp. 171-180. DoI: 10.1002/jmri. 24373 (cit. on p. 40). 
[62] V. Roeloffs, X. Wang, T. J. Sumpf, M. Untenberger, D. Voit, and J. Frahm. "Model-based reconstruction for T1 mapping using single-shot inversionrecovery radial FLASH". International Journal of Imaging Systems and Technology 26.4 (2016), pp. 254-263. DOI: 10.1002 /ima. 22196 (cit. on pp. 43, 96).

[63] J. A. Fessler. "Model-based image reconstruction for MRI". Signal Processing Magazine, IEEE 27.4 (2010), pp. 81-89. DOI: 10.1109/MSP. 2010.936726 (cit. on p. 45).

[64] R. Deichmann. "Fast high-resolution T1 mapping of the human brain". Magnetic Resonance in Medicine 54.1 (2005), pp. 20-27 (cit. on p. 46).

[65] S. Clare and P. Jezzard. "Rapid T1 mapping using multislice echo planar imaging". Magnetic Resonance in Medicine 45.4 (2001), pp. 630-634 (cit. on p. 46).

[66] A. Bakushinsky and M. Kokurin. Iterative methods for approximate solution of inverse problems. Springer Berlin, Heidelberg, 2005 (cit. on p. 46).

[67] A. Doicu, F. Schreier, and M. Hess. "Iteratively regularized Gauss-Newton method for atmospheric remote sensing". Computer Physics Communications 148.2 (2002), pp. 214-226. DOI: $10.1016 / \mathrm{S} 0010-4655$ (02) 00555-6 (cit. on p. 47).

[68] D. Ma, V. Gulani, N. Seiberlich, K. Liu, J. L. Sunshine, J. L. Duerk, and M. A. Griswold. "Magnetic resonance fingerprinting". Nature 495.7440 (2013), pp. 187-192. DOI: 10.1038/nature11971 (cit. on p. 47).

[69] H. Lu, L. M. Nagae-Poetscher, X. Golay, D. Lin, M. Pomper, and P. C. M. Van Zijl. "Routine clinical brain MRI sequences for use at 3.o tesla". Journal of Magnetic Resonance Imaging 22.1 (2005), pp. 13-22. DOI: 10.1002/jmri . 20356 (cit. on p. 54).

[7o] N. Gelman, J. R. Ewing, J. M. Gorell, E. M. Spickler, and E. G. Solomon. "Interregional variation of longitudinal relaxation rates in human brain at 3.o T: Relation to estimated iron and water contents". Magnetic Resonance in Medicine 45.1 (2001), pp. 71-79. DOI: 10 .1002/1522-2594(200101) 45 : 1<71: : AID-MRM1011>3.0.CD;2-2 (cit. on p. 54).

[71] D. C. Zhu and R. D. Penn. "Full-brain T1 mapping through inversion recovery fast spin echo imaging with time-efficient slice ordering". Magnetic Resonance in Medicine 54.3 (2005), pp. 725-731. DOI: 10.1002/mrm . 20602 (cit. on p. 54).

[72] C. Preibisch and R. Deichmann. "Influence of RF spoiling on the stability and accuracy of T1 mapping based on spoiled FLASH with varying flip angles". Magnetic Resonance in Medicine 61.1 (2009), pp. 125-135 (cit. on p. 54). 
[73] J. P. Wansapura, S. K. Holland, R. S. Dunn, and W. S. Ball. "NMR relaxation times in the human brain at 3.0 tesla". Journal of Magnetic Resonance Imaging 9.4 (1999), pp. 531-538 (cit. on p. 54).

[74] M. Haimerl, N. Verloh, F. Zeman, C. Fellner, R. Müller-Wille, A. G. Schreyer, C. Stroszczynski, and P. Wiggermann. "Assessment of Clinical Signs of Liver Cirrhosis Using T1 Mapping on Gd-EOB-DTPA-Enhanced 3T MRI". PLoS ONE 8.12 (2013), e85658. DOI: 10.1371/journal. pone. 0085658 (cit. on p. 54).

[75] C. M. de Bazelaire, G. D. Duhamel, N. M. Rofsky, and D. C. Alsop. "MR imaging relaxation times of abdominal and pelvic tissues measured in vivo at 3.0 T: preliminary results". Radiology 230.3 (2004), pp. 652-659. DOI: 10.1148/radiol.2303021331 (cit. on p. 54).

[76] K. A. Gillis, C. McComb, J. E. Foster, A. H. M. Taylor, R. K. Patel, S. T. W. Morris, A. G. Jardine, M. P. Schneider, G. H. Roditi, C. Delles, and P. B. Mark. "Inter-study reproducibility of arterial spin labelling magnetic resonance imaging for measurement of renal perfusion in healthy volunteers at 3 Tesla". BMC Nephrology 15.1 (2014), pp. 15-23. DOI: 10.1186/1471-236915-23 (cit. on p. 54).

[77] P. Mojabi and J. LoVetri. "Overview and classification of some regularization techniques for the Gauss-Newton inversion method applied to inverse scattering problems". IEEE Transactions on Antennas and Propagation 57.9 (2009), pp. 2658-2665. DOI: 10.1109/TAP. 2009. 2027161 (cit. on p. 57).

[78] D. F. McGivney, E. Pierre, D. Ma, Y. Jiang, H. Saybasili, V. Gulani, and M. A. Griswold. "SVD Compression for Magnetic Resonance Fingerprinting in the Time Domain". IEEE Transactions on Medical Imaging 33.12 (2014), pp. 2311-2322 (cit. on p. 59).

[79] T. K. Jensen. "Stabilization Algorithms for Large-Scale Problems". PhD Thesis. Technical University of Denmark, 2006 (cit. on p. 59).

[8o] M. Uecker, S. Zhang, and J. Frahm. "Nonlinear inverse reconstruction for real-time MRI of the human heart using undersampled radial FLASH". Magnetic Resonance in Medicine 63.6 (2010), pp. 1456-1462. DoI: 10.1002 / mrm. 22453 (cit. on p. 6o).

[81] F. Wajer and K. P. Pruessmann. "Major speedup of reconstruction for sensitivity encoding with arbitrary trajectories". In: Proceedings of the gth Annual Meeting of the ISMRM, Glasgow, Scotland. 2001, p. 767 (cit. on p. 60).

[82] J. Kollmeier. "Perfusion Phantom Studies using Real-Time Magnetic Resonance Imaging". Master Thesis (in preparation). University of Goettingen, 2016 (cit. on pp. 63, 70, 76). 
[83] M. Notohamiprodjo, M. F. Reiser, and S. P. Sourbron. "Diffusion and perfusion of the kidney". European Journal of Radiology 76.3 (2010), pp. 337-347. DOI: $10.1016 / j$.ejrad.2010.05.033 (cit. on p. 63).

[84] Y. Gordon, S. Partovi, M. Müller-Eschner, E. Amarteifio, T. Bäuerle, M.-A. Weber, H.-U. Kauczor, and F. Rengier. "Dynamic contrast-enhanced magnetic resonance imaging: fundamentals and application to the evaluation of the peripheral perfusion". Cardiovascular Diagnosis and Therapy 4.2 (2014), pp. 147-164. DOI: 10.3978/j.issn.2223-3652.2014.03.01 (cit. on p. 63).

[85] C.-H. Wang, F.-F. Yin, J. Horton, and Z. Chang. "Review of treatment assessment using DCE-MRI in breast cancer radiation therapy". World Journal of Methodology 4.2 (2014), pp. 46-58. DOI: 10.5662/wjm. v4.i2.46 (cit. on p. 63).

[86] S. Verma, B. Turkbey, N. Muradyan, A. Rajesh, F. Cornud, M. A. Haider, P. L. Choyke, and M. Harisinghani. "Overview of Dynamic Contrast-Enhanced MRI in Prostate Cancer Diagnosis and Management". American Journal of Roentgenology 198.6 (2012), pp. 1277-1288. DOI: 10.2214/AJR. 12.8510 (cit. on p. 63).

[87] M. Bergamino, L. Bonzano, F. Levrero, G. L. Mancardi, and L. Roccatagliata. "A review of technical aspects of T1-weighted dynamic contrast-enhanced magnetic resonance imaging (DCE-MRI) in human brain tumors". Physica Medica 30.6 (2014), pp. 635-643. DOI: 10.1016/j . ejmp. 2014.04 .005 (cit. on p. 63).

[88] G. Brix, W. Semmler, R. Port, L. R. Schad, G. Layer, and W. J. Lorenz. "Pharmacokinetic parameters in CNS Gd-DTPA enhanced MR imaging". Journal of Computer Assisted Tomography 15.4 (1991), pp. 621-628 (cit. on p. 63).

[89] D. L. Buckley, R. W. Kerslake, S. J. Blackband, and A. Horsman. "Quantitative analysis of multi-slice Gd-DTPA enhanced dynamic MR images using an automated simplex minimization procedure". Magnetic Resonance in Medicine 32.5 (1994), pp. 646-651. DoI: $10.1002 / \mathrm{mrm} .1910320514$ (cit. on p. 63).

[9o] U. Hoffmann, G. Brix, M. V. Knopp, T. Hess, and W. J. Lorenz. "Pharmacokinetic mapping of the breast: A new method for dynamic MR mammography". Magnetic Resonance in Medicine 33.4 (1995), pp. 506-514. DOI: 10.1002/mrm. 1910330408 (cit. on p. 63).

[91] A. Freeman, P. Gowland, and P. Mansfield. “Optimization of the ultrafast look-locker echo-planar imaging T1 mapping sequence". Magnetic Resonance Imaging 16.7 (1998), pp. 765-772. DOI: 10 . 1016/S0730-725X (98) 00011-3 (cit. on p. 64). 
[92] P. S. Tofts and A. G. Kermode. "Measurement of the blood-brain barrier permeability and leakage space using dynamic MR imaging. 1. Fundamental concepts". Magnetic Resonance in Medicine 17.2 (1991), pp. 357-367. DoI: 10.1002/mrm. 1910170208 (cit. on pp. 64, 68).

[93] B. Driscoll, H. Keller, and C. Coolens. "Development of a dynamic flow imaging phantom for dynamic contrast-enhanced CT". Medical Physics 38.8 (2011), pp. 4866-488o (cit. on p. 64).

[94] B. Driscoll, H. Keller, D. Jaffray, and C. Coolens. "Development of a dynamic quality assurance testing protocol for multisite clinical trial DCE-CT accreditation". Medical Physics 40.8 (2013), p. 081906. DoI: 10.1118/1. 4812429 (cit. on p. 64).

[95] M. Peladeau-Pigeon and C. Coolens. "Computational fluid dynamics modelling of perfusion measurements in dynamic contrast-enhanced computed tomography: development, validation and clinical applications". Physics in Medicine and Biology 58.17 (2013), pp. 6111-6131. DOI: 10 . 1088/00319155/58/17/6111 (cit. on p. 64).

[96] S. L. Lee, W. Foltz, B. Driscoll, A. Fatemi, C. Menard, C. Coolens, and C. Chung. "Comparison of arterial input functions by magnitude and phase signal measurement in dynamic contrast enhancement MRI using a dynamic flow phantom". In: Proceedings of the 2oth Annual Meeting of ISMRM. Melbourne, Australia. 2012, p. 236 (cit. on p. 64).

[97] F. Khalifa, A. Soliman, A. El-Baz, M. Abou El-Ghar, T. El-Diasty, G. Gimel'farb, R. Ouseph, and A. C. Dwyer. "Models and methods for analyzing DCEMRI: a review". Medical Physics 41.12 (2014), p. 124301. DOI: 10.1118/1. 4898202 (cit. on pp. 65, 68).

[98] P. S. Tofts, G. Brix, D. L. Buckley, J. L Evelhoch, E. Henderson, M. V. Knopp, H. B. W. Larsson, T.-Y. Lee, N. a. Mayr, G. J. M. Parker, R. E. Port, J. Taylor, and R. M. Weisskoff. "Estimating Kinetic Parameters From Dynamic Contrast-Enhanced T1-Weighted MRI of a Diffusable Tracer: Standardized Quantities and Symbols". Journal of Magnetic Resonance Imaging 10.3 (1999), pp. 223-232 (cit. on pp. 65, 68).

[99] P. S. Tofts. "Modeling Tracer Kinetics in Dynamic Gd-DTPA MR Imaging". Journal of Magnetic Resonance Imaging 7.1 (1997), pp. 91-101 (cit. on p. 68).

[100] N. Bloembergen. "Proton Relaxation Times in Paramagnetic Solutions". The Journal of Chemical Physics 27.2 (1957), p. 572. DOI: 10.1063/1.1743771 (cit. on p. 70$)$. 
[101] G. J. Stanisz and R. M. Henkelman. “Gd-DTPA relaxivity depends on macromolecular content". Magnetic Resonance in Medicine 44.5 (2000), pp. 665-667. DOI: 10 . 1002/1522-2594(200011) $44: 5<665:$ : AID - MRM1 > 3 . 0 . C0 ; 2-M (cit. on p. 70).

[102] T. E. Yankeelov, W. D. Rooney, X. Li, and C. S. Springer. "Variation of the Relaxographic "Shutter-Speed" for Transcytolemmal Water Exchange Affects the CR Bolus-Tracking Curve Shape". Magnetic Resonance in Medicine 50.6 (2003), pp. 1151-1169. DOI: 10.1002/mrm. 10624 (cit. on p. 70).

[103] L. Ostergaard, R. M. Weisskoff, D. A. Chesler, C. Gyldensted, and B. R. Rosen. "High resolution measurement of cerebral blood flow using intravascular tracer bolus passages. Part I: Mathematical approach and statistical analysis". Magnetic Resonance in Medicine 36.5 (1996), pp. 715-725. DOI: $10.1002 / \mathrm{mrm} .1910360510$ (cit. on p. 72).

[104] R. Deichmann, D. Hahn, and A. Haase. "Fast T1 Mapping on a Whole-Body Scanner". Magnetic Resonance in Medicine 209.1 (1999), pp. 206-209 (cit. on p. 75).

[105] P. Kellman and M. S. Hansen. "T1-mapping in the heart: accuracy and precision". Journal of Cardiovascular Magnetic Resonance 16.1 (2014), p. 2. DOI: 10.1186/1532-429X-16-2 (cit. on p. 76).

[106] T.-L. Hwang, P. C. van Zijl, and M. Garwood. "Fast Broadband Inversion by Adiabatic Pulses". Journal of Magnetic Resonance 133.1 (1998), pp. 200-203. DOI: 10.1006/jmre.1998.1441 (cit. on p. 76).

[107] A. P. Crawley, M. L. Wood, and R. M. Henkelman. "Elimination of transverse coherences in FLASH MRI". Magnetic Resonance in Medicine 8.3 (1988), pp. 248-26o (cit. on p. 77).

[108] X. Wang, A. A. Joseph, O. Kalentev, K.-D. Merboldt, D. Voit, V. B. Roeloffs, M. van Zalk, and J. Frahm. "High-resolution myocardial T 1 mapping using single-shot inversion recovery fast low-angle shot MRI with radial undersampling and iterative reconstruction". The British Journal of Radiology 89.1068 (2016), p. 20160255. DOI: 10.1259/bjr. 20160255 (cit. on p. 96).

[109] P. Schuenke, J. Windschuh, V. Roeloffs, M. E. Ladd, P. Bachert, and M. Zaiss. "Simultaneous mapping of water shift and B1 (WASABI)-Application to field-inhomogeneity correction of CEST MRI data". Magnetic Resonance in Medicine (2016). DOI: 10.1002/mrm. 26133 (cit. on p. 96).

[110] O. Bodet, S. Goerke, N. G. R. Behl, V. Roeloffs, M. Zaiss, and P. Bachert. "Amide proton transfer of carnosine in aqueous solution studied in vitro by WEX and CEST experiments". NMR in Biomedicine 28.9 (2015), pp. 10971103. DOI: $10.1002 / \mathrm{nbm} .3343$ (cit. on p. 96). 
[111] M. Untenberger, Z. Tan, D. Voit, A. A. Joseph, V. Roeloffs, K. D. Merboldt, S. Schätz, and J. Frahm. "Advances in real-time phase-contrast flow MRI using asymmetric radial gradient echoes". Magnetic Resonance in Medicine (2015). DOI: 10.1002/mrm. 25696 (cit. on p. 96).

[112] S. Hofer, X. Wang, V. Roeloffs, and J. Frahm. "Single-shot T1 mapping of the corpus callosum: a rapid characterization of fiber bundle anatomy". Frontiers in Neuroanatomy 9 (2015), pp. 1-6. DOI: 10.3389/fnana.2015.00057 (cit. on p. 96).

[113] V. Roeloffs, C. Meyer, P. Bachert, and M. Zaiss. "Towards quantification of pulsed spinlock and CEST at clinical MR scanners: an analytical interleaved saturation-relaxation (ISAR) approach". NMR in Biomedicine 28.1 (2014), pp. 40-53. DOI: 10.1002/nbm.3192 (cit. on p. 96). 


\section{ACRONYMS}

\begin{tabular}{ll} 
AIF & arterial input function \\
ASL & arterial spin labeling \\
BSSFP & balanced steady-state free precession \\
CA & contrast agent \\
CG & conjugate gradients \\
CT & computed tomography \\
CUDA & compute unified device architecture \\
DCE & dynamic contrast-enhanced \\
DSC & dynamic susceptibility contrast-enhanced \\
DTI & diffusion tensor imaging \\
EES & extravascular extracellular space \\
EIS & extravascular intracellular space \\
EPI & echo planar imaging \\
ETM & extended Tofts model \\
FFT & fast Fourier transform \\
FID & free induction decay \\
FLASH & fast low-angle shot \\
FSE & fast spin echo \\
FOV & field of view \\
GPU & graphics processing unit \\
IRGN & iteratively regularized Gauss-Newton method \\
IR & inversion recovery \\
\hline
\end{tabular}




\begin{tabular}{|c|c|}
\hline LL & Look-Locker \\
\hline MEX & MATLAB executable \\
\hline MRI & magnetic resonance imaging \\
\hline MR & magnetic resonance \\
\hline NLINV & nonlinear inversion \\
\hline PET & positron emission tomography \\
\hline PSF & point spread function \\
\hline RARE & rapid acquisition with refocused echoes \\
\hline RF & radiofrequency \\
\hline ROI & region of interest \\
\hline SD & standard deviation \\
\hline SENSE & sensitivity encoding \\
\hline SNR & signal-noise ratio \\
\hline SSFP & steady-state free precession \\
\hline SPECT & single photon emission tomography \\
\hline $\mathrm{TE}$ & echo time \\
\hline TI & inversion time \\
\hline $\mathrm{TM}$ & Tofts model \\
\hline TR & repetition time \\
\hline
\end{tabular}




\section{CURRICULUM VITAE}

VOLKERT BRAR ROELOFFS

18.09.1987 Born in Wyk auf Föhr

SCIENTIFIC EDUCATION:

SINCE 2012 PhD student

with Prof. Dr. Jens Frahm (advisor)

in the International Max Planck Research School (IMPRS)

in the program of Physics of Biological and Complex Systems (GGNB)

at the Georg-August-Universität Göttingen, Germany

$2011 \quad$ Research visit

Case Western Reserve University, Cleveland, USA

CCIR/MRI research group under Prof. M. Griswold/Prof. J. Duerk

2010-2012 Master's degree in Physics

Examination: Grade "1.0" (very good)

with Prof. Dr. Peter Bachert (advisor), German Cancer Research Center at the Ruprecht-Karls-Universität Heidelberg, Germany

Thesis title: “1H-NMR Magnetization Transfer Experiments with

Pulsed Off-Resonant Spin-Locking under Chemical Exchange"

2007-2010 Bachelor's degree in Physics

Examination: Grade "1.4" (very good)

with Prof. Dr. Lothar Schad (advisor), Medical Faculty Mannheim at the Ruprecht-Karls-Universität Heidelberg, Germany

Thesis title: “Development of a ${ }^{1} \mathrm{H} /{ }^{19} \mathrm{~F}-\mathrm{Coil}$ for Quantification

of Substance via NMR Spectroscopy at $9.4 \mathrm{~T}^{\prime \prime}$

$2007 \quad$ Internship

Max Planck Institute for Plasma Physics (IPP), Garching

1997-2006 Gymnasium Insel Föhr

Abitur (Graduation): Grade “1.o" (very good) 
PUBLICATIONS:

2016 V. Roeloffs, X. Wang, T. J. Sumpf, M. Untenberger, D. Voit, and J. Frahm. "Modelbased reconstruction for $\mathrm{T}_{1}$ mapping using single-shot inversion-recovery radial FLASH". International Journal of Imaging Systems and Technology 26.4 (2016), pp. 254263. DOI: $10.1002 /$ ima. 22196

2016 X. Wang, A. A. Joseph, O. Kalentev, K.-D. Merboldt, D. Voit, V. B. Roeloffs, M. van Zalk, and J. Frahm. "High-resolution myocardial T 1 mapping using singleshot inversion recovery fast low-angle shot MRI with radial undersampling and iterative reconstruction". The British Journal of Radiology 89.1068 (2016), p. 20160255. DOI: $10.1259 / \mathrm{bjr} .20160255$

2016 P. Schuenke, J. Windschuh, V. Roeloffs, M. E. Ladd, P. Bachert, and M. Zaiss. "Simultaneous mapping of water shift and B1 (WASABI)-Application to field-inhomogeneity correction of CEST MRI data". Magnetic Resonance in Medicine (2016). DOI: 10.1002/ mrm. 26133

2016 Z. Tan, V. Roeloffs, D. Voit, A. A. Joseph, M. Untenberger, K. D. Merboldt, and J. Frahm. "Model-based reconstruction for real-time phase-contrast flow MRI: Improved spatiotemporal accuracy". Magnetic Resonance in Medicine (Mar. 2016). DoI: $10.1002 / \mathrm{mrm} .26192$

2015 O. Bodet, S. Goerke, N. G. R. Behl, V. Roeloffs, M. Zaiss, and P. Bachert. "Amide proton transfer of carnosine in aqueous solution studied in vitro by WEX and CEST experiments". NMR in Biomedicine 28.9 (2015), pp. 1097-1103. DoI: 10.1002/nbm . 3343

2015 M. Untenberger, Z. Tan, D. Voit, A. A. Joseph, V. Roeloffs, K. D. Merboldt, S. Schätz, and J. Frahm. "Advances in real-time phase-contrast flow MRI using asymmetric radial gradient echoes". Magnetic Resonance in Medicine (2015). DOI: 10.1002/mrm . 25696

2015 V. Roeloffs, D. Voit, and J. Frahm. "Spoiling without additional gradients: Radial FLASH MRI with randomized radiofrequency phases". Magnetic Resonance in Medicine (June 2015). DOI: 10.1002/mrm. 25809

2015 S. Hofer, X. Wang, V. Roeloffs, and J. Frahm. "Single-shot T1 mapping of the corpus callosum: a rapid characterization of fiber bundle anatomy". Frontiers in Neuroanatomy 9 (May 2015), pp. 1-6. DoI: 10.3389/fnana.2015.00057

2015 X. Wang, V. Roeloffs, K. D. Merboldt, D. Voit, and J. Frahm. "Single-shot Multi-slice T1 Mapping at High Spatial Resolution - Inversion-Recovery FLASH with Radial Undersampling and Iterative Reconstruction". Open Medical Imaging Journal (2015), pp. $1-8$

2014 V. Roeloffs, C. Meyer, P. Bachert, and M. Zaiss. "Towards quantification of pulsed spinlock and CEST at clinical MR scanners: an analytical interleaved saturationrelaxation (ISAR) approach". NMR in Biomedicine 28.1 (2014), pp. 40-53. DOI: 10 . 1002/nbm. 3192 
CONFERENCES AND CONTRIBUTIONS:

2016 Annual Meeting of the ISMRM, Singapore

Travel Grant (GGNB-PBCS) + Educational Stipend ISMRM

Electronic Poster: "Advances in Model-Based Reconstruction of High Resolution $T_{1}$ maps"

2015 Annual Meeting of the ISMRM, Toronto, Canada

Travel Grant (GGNB-PBCS) + Educational Stipend ISMRM

Electronic Poster: "High resolution $T_{1}$ mapping within Seconds: Model-based Reconstruction without Regularization"

2014 Joint Annual Meeting of the ISMRM-ESMRMB, Milan, Italy

2013 GGNB-Science Day, Göttingen

Traditional Poster: "Measure Less, Get the Same: Model-Based Parameter Mapping in Magnetic Resonance Imaging"

2013 Annual Meeting of the ISMRM, Salt Lake City, USA

Travel Grant (GGNB-PBCS)

Traditional Poster: "An Analytical Approach towards pulsed-SL/CEST Quantification"

2012 Annual Meeting DGMP, Jena, Germany

Talk: "An Analytical Approach towards pulsed-SL/CEST Quantification"

2010 Annual Meeting Deutsche Sektion ISMRM, Essen, Germany

Travel Grant (DS-ISMRM)

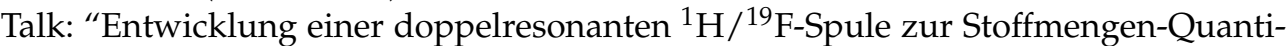
fizierung mittels Magnetresonanzspektroskopie bei $9.4 \mathrm{~T}^{\prime \prime}$

COURSES AND WORKSHOPS:

2014 16. Doktorandentraining der DS-ISMRM, Würzburg

Travel Grant (GGNB-PBCS)

"Quantitative MRT"

2013 ESMRMB Lectures, Freiburg

"Inverse imaging, sparse sampling, compressed sensing, and more"

2013 GGNB Short Methods Course, Göttingen

"Introduction to molecular dynamic simulation"

2013 GGNB Short Methods Course, Göttingen

"EPR-Spectroscopy" 
TEACHING EXPERIENCE:

2015-2016 Supervision of master thesis project (Jost Kollmeier)

"Perfusion Phantom Studies using Real-Time

Magnetic Resonance Imaging"

2013-2015 Several tutorials, including lectures and hands-on sessions for graduate students

"Basic Principles of MRI and Real-time MRI"

2013-2015 "Zukunftstag" (tutorial with hands-on experiments for school students)

"Magnetresonanztomographie"

2013 Lecture-accompanying exercise group leader

"Physik IV: Quantenphysik, Atom- und Molekülphysik"

LANGUAGES:

Frisian/German (bilingual native)

English

Latin ("großes Latinum")

French (CEFR Level B2) 
COLOPHON

This document was typeset in $\mathrm{LT}_{\mathrm{E}} \mathrm{X}$ using the typographical look-and-feel classicthesis. The bibliography was typeset using biblatex. 